\title{
WestVirginiaUniversity
}

THE RESEARCH REPOSITORY @ WVU

Graduate Theses, Dissertations, and Problem Reports

2018

\section{Finding Booth: My Journey through TopdogUnderdog}

Andra Ward Jr.

Follow this and additional works at: https://researchrepository.wvu.edu/etd

\section{Recommended Citation}

Ward Jr., Andra, "Finding Booth: My Journey through TopdogUnderdog" (2018). Graduate Theses, Dissertations, and Problem Reports. 6915.

https://researchrepository.wvu.edu/etd/6915

This Thesis is protected by copyright and/or related rights. It has been brought to you by the The Research Repository @ WVU with permission from the rights-holder(s). You are free to use this Thesis in any way that is permitted by the copyright and related rights legislation that applies to your use. For other uses you must obtain permission from the rights-holder(s) directly, unless additional rights are indicated by a Creative Commons license in the record and/ or on the work itself. This Thesis has been accepted for inclusion in WVU Graduate Theses, Dissertations, and Problem Reports collection by an authorized administrator of The Research Repository @ WVU. For more information, please contact researchrepository@mail.wvu.edu. 


\title{
Empirical Genetic Algorithm Parameter Tuning Concerning the Synthesis of Combinational Logic Circuits
}

\author{
Joshua Ward \\ Thesis submitted to the \\ Lane Department of Computer Science and Electrical Engineering \\ at West Virginia University
}

in partial fulfillment of the requirements for the degree of Master of Science in Electrical Engineering

\author{
Roy Nutter, Ph.D., Chair \\ Matthew Valenti, Ph.D. \\ Yanfang Ye, Ph.D.
}

Lane Department of Computer Science and Electrical Engineering

\author{
Morgantown, West Virginia
}

2017

Keywords: Genetic Algorithm, Combinational Logic Circuit, Parameter Tuning, Evolutionary Algorithm

Copyright 2017 Joshua Ward 


\title{
Abstract \\ Empirical Genetic Algorithm Parameter Tuning Concerning the Synthesis of Combinational Logic Circuits
}

\author{
Joshua Ward
}

This study identifies optimal parameter values for a genetic algorithm used in the synthesis of combinational logic circuits. Parameters used to obtain an initial solution circuit are examined as well as parameters used to optimize solution circuits. Finding an initial solution circuit was done using a fitness function that was less influential while optimizing solution circuits used a stronger fitness function. This allowed parameters to be studied under very different circumstances. It was discovered that parameters which maintained a higher genetic diversity and strayed farther from the concept of "Survival of the Fittest" produced better results in general. 


\section{Acknowledgments}

I would like to thank my friends and family, especially my mother, father, and loving lady, Margot, Randall, and Annie, for keeping me sane on my journey through graduate school. I would also like to thank my committee members, Dr. Matthew Valenti and Dr. Yanfang Ye for offering their valuable time to help me along the way. I would especially like to thank my committee chair, Dr. Roy Nutter, for everything he has done for me throughout my graduate career. Between helping me find funding, offering words of wisdom, or calming me down with kind words when he saw I was stressed out of my mind, he's been there to help every step of the way. Finally, I would like to thank the big Man upstairs. My faith has empowered me to find the end of this long and winding road.

I can do all things through Christ who strengthens me. 


\section{Table of Contents}

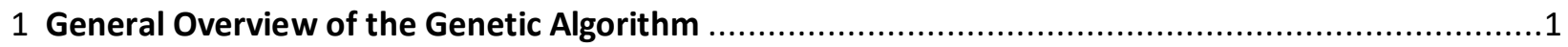

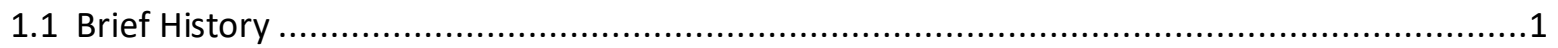

1.2 What is a Genetic Algorithm? ..........................................................................

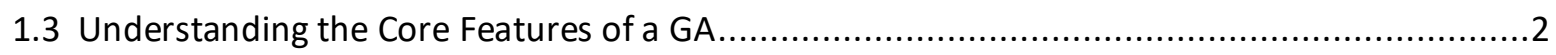

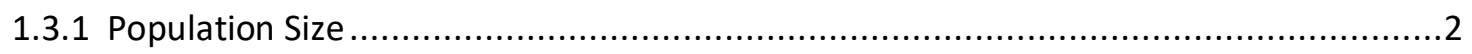

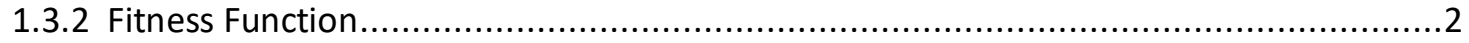

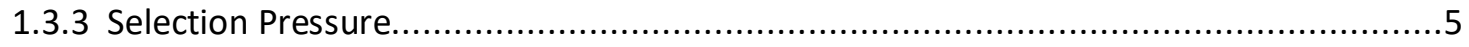

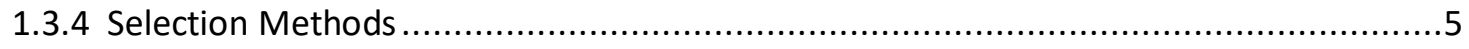

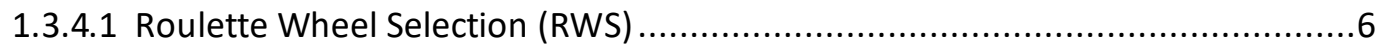

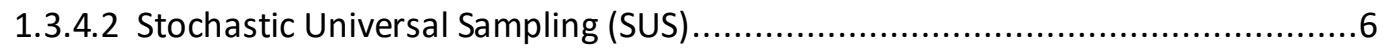

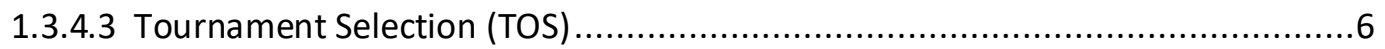

1.3.4.4 Linear/Exponential Ranking Selection.................................................

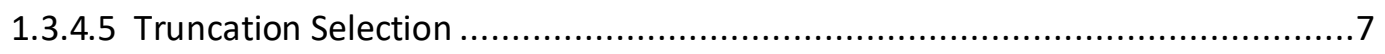

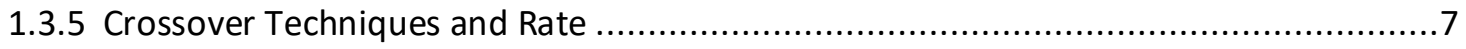

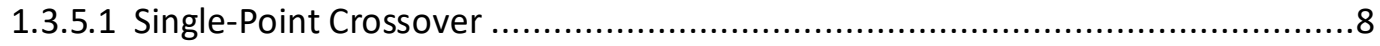

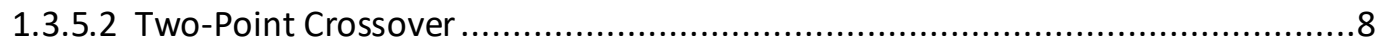

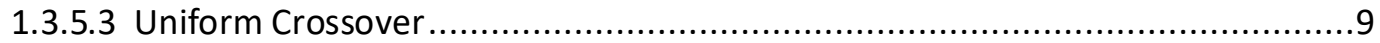

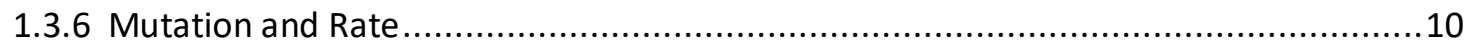

1.3.6.1 Maintaining Genetic Diversity............................................................ 10

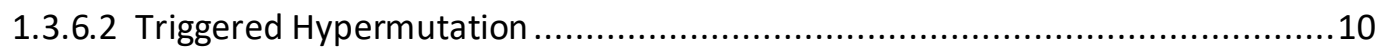

1.3.6.3 Random Immigrants................................................................... 11

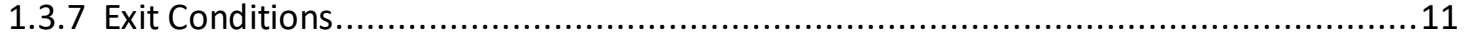

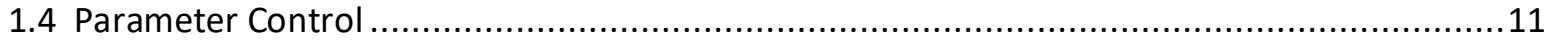

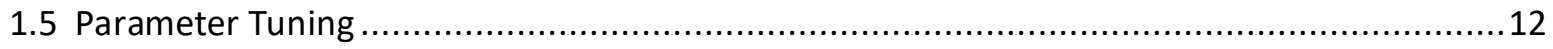

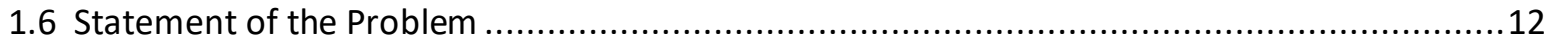

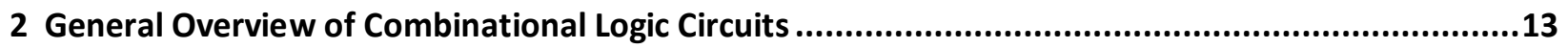

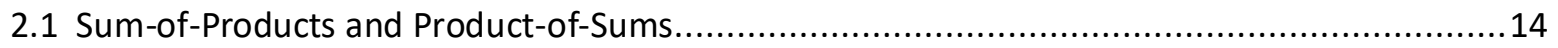

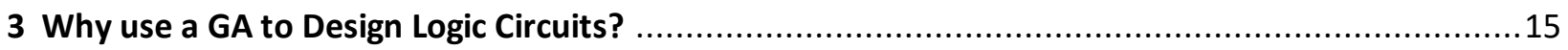

3.1 Human Design Space vs. Evolutionary Computation Methods ....................................15

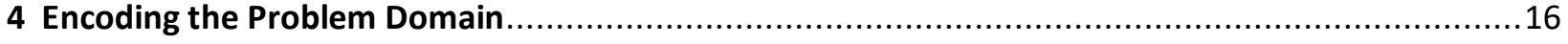

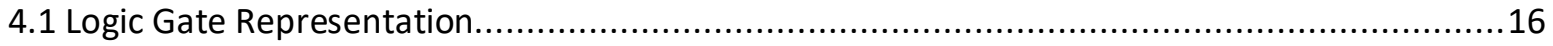

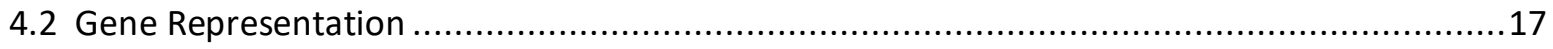




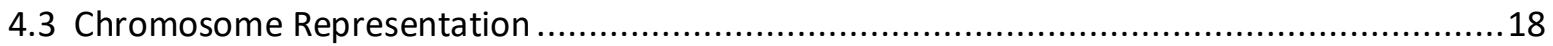

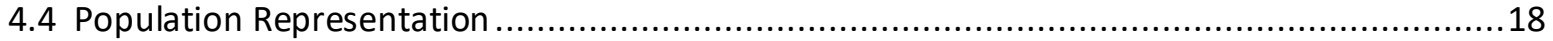

5 Fitness Function Disparity Between Solutions and Non-Solutions ........................................19

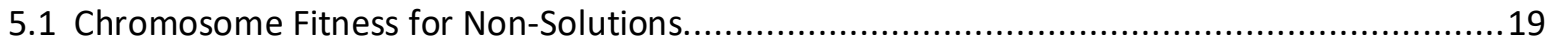

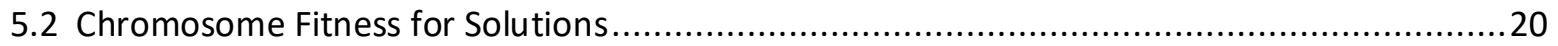

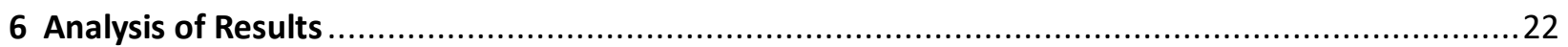

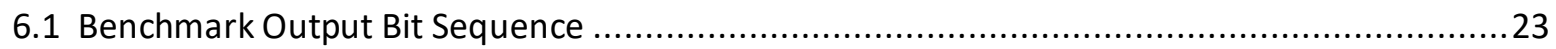

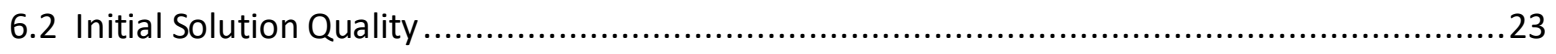

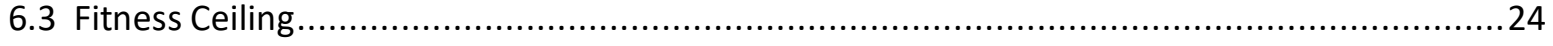

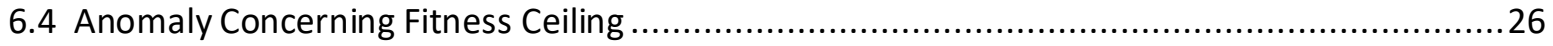

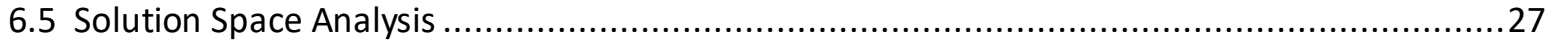

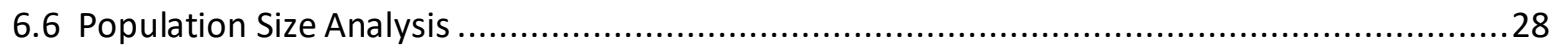

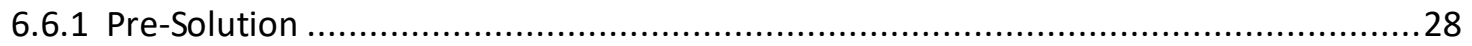

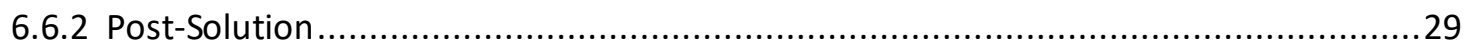

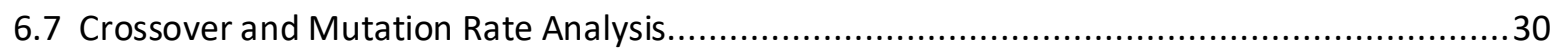

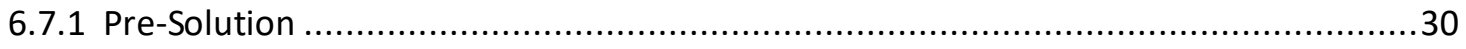

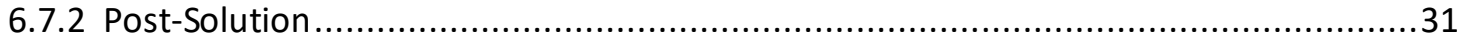

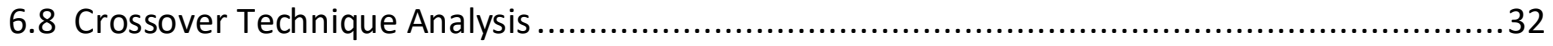

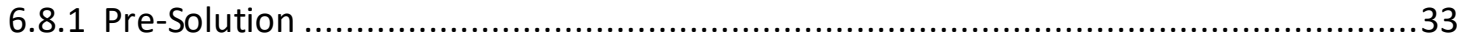

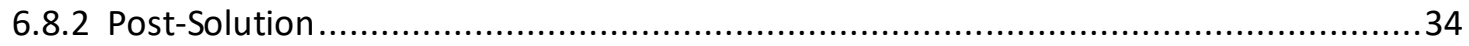

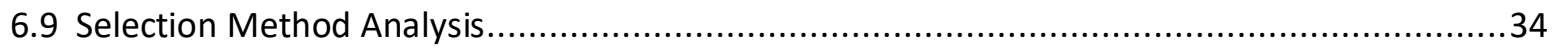

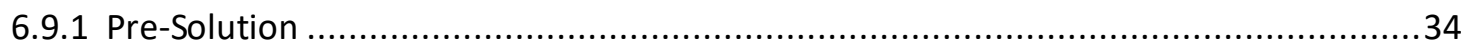

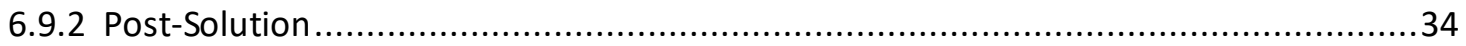

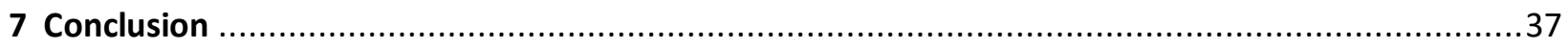

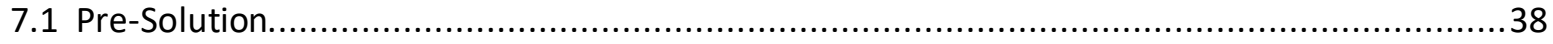

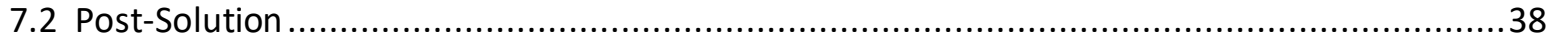

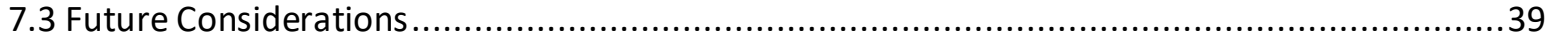

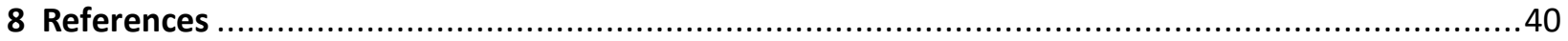

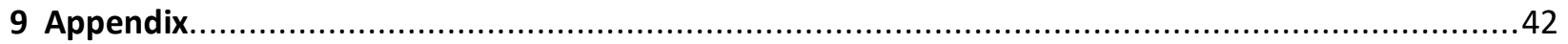

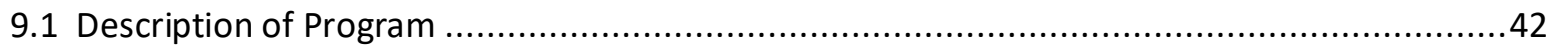

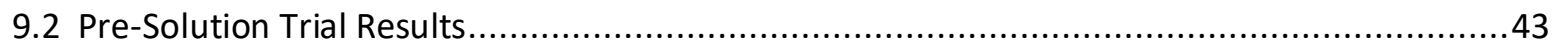

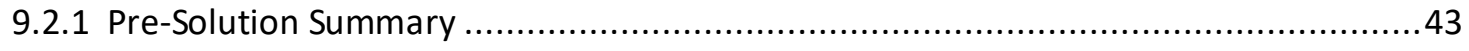

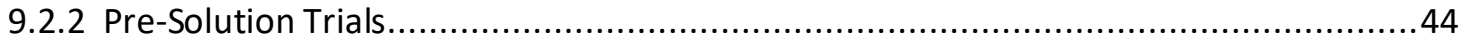




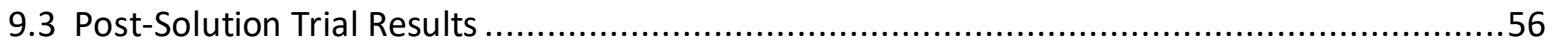

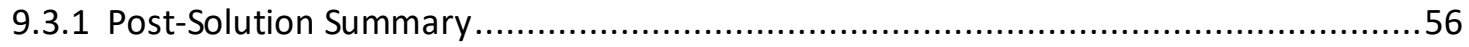

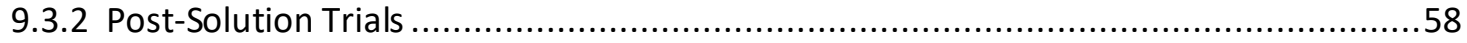




\section{General Overview of the Genetic Algorithm}

\subsection{Brief History}

The beginnings of the Genetic Algorithm began in the 1950s and 1960s when several computer scientists independently studied evolutionary systems with the idea that evolution could be used as an optimization tool for engineering problems. The basic premise in these systems was to evolve a population of candidate solutions using operators inspired by natural selection and genetic variation [1].

In the 1960s, genetic algorithms were formally introduced by John Holland. They were developed by Holland and his students and colleagues at the University of Michigan. Their goal was to study the phenomenon of adaptation as it occurs in nature and to develop methods of importing the mechanisms of natural adaptation into computer systems [1].

\subsection{What is a Genetic Algorithm?}

A Genetic Algorithm (GA) is a type of evolutionary algorithm (EA) that is typically based on the concept of natural selection. Most evolutionary computation methods that are considered to be GAs have a few things in common: populations of chromosomes which are made up of genes, selection based on fitness, crossover to create new offspring, and possible mutation of the offspring [1]. GAs begin with a randomly generated initial population of chromosomes. A fitness check is performed on each chromosome in the population which acts as the driving force for the selection method. Different selection methods have different reliance upon the fitness function, but their job is to select the best candidates for crossover. Good selection methods accomplish this while still maintaining the ability to allow less fit individuals to be considered 
for crossover which limits premature convergence. The likelihood of crossing over or mutating candidate chromosomes is based on a crossover rate and mutation rate respectively. The offspring of the resulting crossovers and mutations are carried into a new generation of chromosomes and the cycle repeats until a particular exit condition is met.

\subsection{Understanding the Core Features of a GA}

There are many parameters that can affect the performance of a genetic algorithm and it is widely acknowledged in the evolutionary computing field that good parameter values are very important to achieve good performance. Unfortunately, there has been very little effort to study the effects these parameters have on the overall performance of the algorithm [8]. Understanding how each piece can affect the performance of the algorithm is crucial to the overall design.

\subsubsection{Population Size}

The general consensus regarding population size is that a "small" population could lead the algorithm to poor solutions, while a large population could force the algorithm to spend an unreasonable amount of time on computation [2].

\subsubsection{Fitness Function}

The fitness function is used to summarize and assign, as a single figure of merit, the "goodness" of any particular candidate solution. This figure is known as the fitness of the chromosome. Typically, a candidate solution is modeled as a chromosome and every chromosome in the population is assigned a fitness. The fitness function can play a very large or very small role 
depending on how much weight the function assigns to any particular chromosome relative to other chromosomes in the population.

Most selection methods choose chromosomes for crossover based on their fitness. It is difficult to understand the importance of the fitness function without understanding the behavior of selection methods as well, but as a general rule, if chromosome $\mathrm{A}$ is assigned a fitness that is much larger than chromosome B, it will be considered much more heavily for crossover. A discussion of selection methods can be found in section 1.3.3.

It is important to recognize that the fitness function isn't necessarily a roadmap to a solution, but rather nudges the GA in the right direction with supplemental information from a human designer. It should not "bully" the GA towards a solution or else premature convergence is a risk that becomes more prominent. This is especially true if the designer is not a subject matter expert regarding the problem domain. The less expertise a designer has with the subject, the more perplexing and frustrating the results can seem if the GA is pushed toward a solution too quickly.

The GA relies more heavily on its inherently random nature as it attempts to converge on an acceptable solution. Where the GA truly excels is its ability to make solutions better once an acceptable solution is achieved. Alluding to the overall topic of this study, synthesis of logic circuits, it is very difficult to design a fitness function for such a problem before a solution is found. For instance, consider that the GA is attempting to build a logic circuit that solves for a particular output bit sequence. It sounds acceptable to award chromosomes based on the number of correct output bits, but what if the GA encounters a chromosome that is the polar opposite of the bit sequence that is being searched for? Obviously this is very close to the desired solution, only a NOT gate away, but the fitness function would quickly throw this chromosome out 
because it was designed that way. Human designers often cannot predict all scenarios that the GA will encounter. It is likely a better idea to allow the GA's random nature to be more prominent while attempting to find a solution. This is also known is having a lower selection pressure. A discussion of selection pressure can be found in section 1.3.3.

Once a solution is found, however, the fitness can be spiked. At this point it becomes easier to tell the GA what makes a solution more fit. In the case of logic circuit synthesis, things like the number of logic gates used or the number of NAND gates used, for instance, can be a clear indication of solution quality.

In summary, the fitness function should start a slow ascent toward a solution, allowing the random nature of the GA to shine through. When a solution is found, the fitness function is able to do a better job of informing the GA what makes a good solution. A graph showing the general fitness landscape for this particular example is found in figure 1 below.

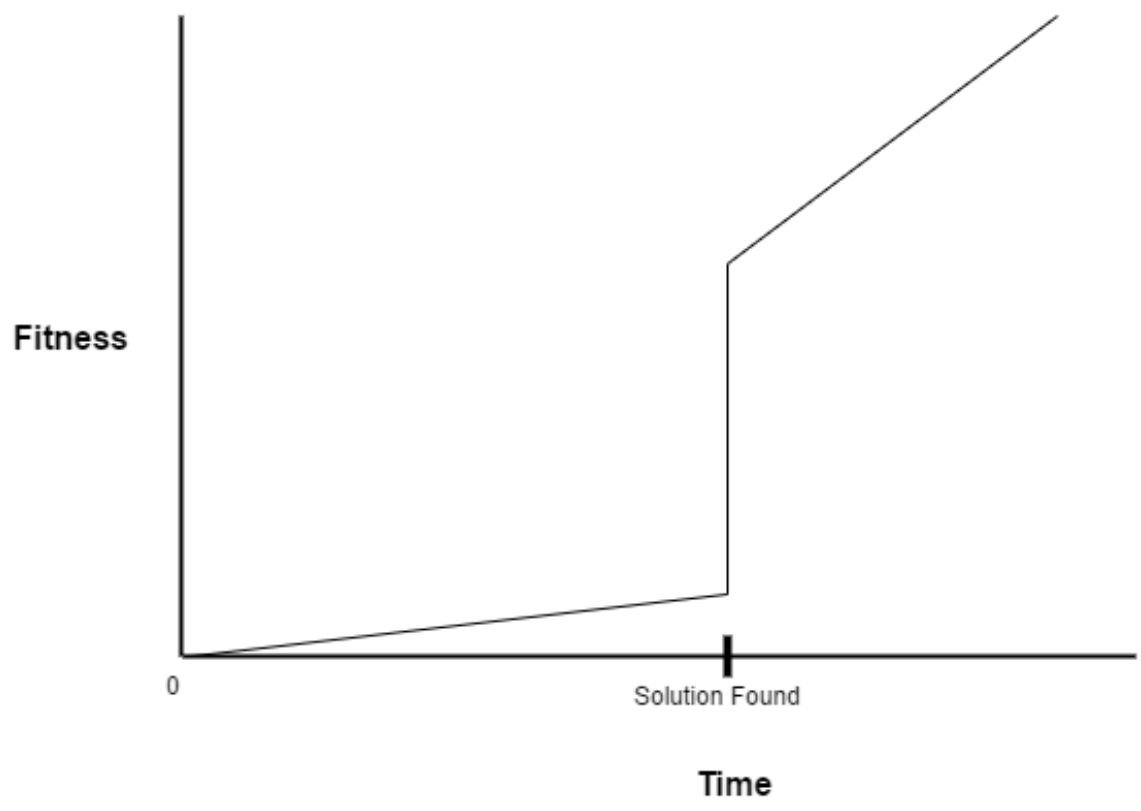

Figure 1 - GA Fitness Landscape 


\subsubsection{Selection Pressure}

Selection pressure is the degree to which higher fitness individuals are favored. Higher selection pressure directly results in higher fitness selections. The rate of convergence for a GA is largely affected by selection pressure as well. Higher selection pressure results in higher convergence rates [20]. It is quite the balancing act to determine an optimal selection pressure especially as it is not typically represented as a numerical value. If the selection pressure is too low, the convergence rate will be slow and the GA will take unnecessarily long to find a solution. Conversely, if the selection pressure is too high, the GA will converge to an incorrect or suboptimal solution [20]. This is also known as premature convergence and is a very wellknown issue for GAs. If it is not clear as to the affect the fitness function is having on the convergence rate or that it is resulting in suboptimal solutions, lowering the selection pressure may be a viable option to achieve better results. A slower solution is better than no solution.

\subsubsection{Selection Methods}

Genetic algorithm selection is the process of choosing chromosomes or individuals to be considered for crossover and mutation. There are a number of commonly acknowledged selection methods that do this in different ways, but they all have some aspect that is reliant on chromosome fitness to some extent. Selection weight is determined based on individual chromosome fitness relative to the population fitness. In general:

$$
\text { selection weight }=\frac{\text { chromosome fitness }}{\text { population fitness }}
$$

where the population fitness is the sum of all individual chromosome fitness. 


\subsubsection{Roulette Wheel Selection (RWS)}

This selection method is also known as Fitness Proportionate Selection. It treats each chromosome in the population as part of a roulette wheel. The odds of a chromosome being selected for crossover are based on the selection weight which is discussed in the previous section. Imagine a roulette wheel being spun and the selection weight of chromosomes dictating the size of their respective portion of the wheel. There are quite a few other selection methods that are based on RWS. One such is Stochastic Universal Sampling.

\subsubsection{Stochastic Universal Sampling (SUS)}

Stochastic Universal Sampling is a variation of RWS, but unlike RWS where there is a single pointer on a spinning wheel that selects a winner, SUS has "N" equally spaced pointers [3]. Because SUS is able to make more selections in a single phase, it is capable of reducing computation time.

\subsubsection{Tournament Selection (TOS)}

Tournament Selection chooses individuals for crossover by holding tournaments with "N" competitors. The winner of the tournament is the individual with the highest fitness or selection weight and will be considered for crossover [20].

It is easier to tune selection pressure when using TOS because the pressure directly correlates to the tournament size while other selection methods are more dependent on adjusting chromosome fitness. For example, a tournament size of $\mathrm{N}=1$ would be equivalent to random selection while a tournament size of $\mathrm{N}=50$ could be considered to have high selection pressure. 


\subsubsection{Linear/Exponential Ranking Selection}

Linear Ranking Selection (LRS) and Exponential Ranking Selection (ERS) are both variations of RWS that were not included in this study and therefore will not be discussed at length. One of the possible issues in calculating selection weight of a chromosome relative to the rest of the population is that it has the chance of severely limiting diversity in the population depending on how large the fitness gaps between individuals can get. For example, if a particular chromosome has a fitness that results in a selection weight of $90 \%$ of the roulette wheel, other chromosomes will have very little chance of being considered for crossover [5]. Instead of calculating selection weight in this way, LRS and ERS assign ranks to chromosomes. They are essentially the same except that they calculate the weight of the ranks differently.

\subsubsection{Truncation Selection}

Truncation Selection is a more simplistic selection method that is not often used in practice as it is prone to premature convergence. It orders candidate solutions according to fitness and then only a portion of the fittest individuals are chosen for crossover. This may still be a viable selection method for problems that use very large populations [4].

\subsubsection{Crossover Techniques and Rate}

The crossover operator of a GA is what is responsible for the recombination of genes from any pair of chosen chromosomes within a population. The crossover rate represents the percent chance of the crossover operator being performed on the selected mating pair. Single-point, twopoint, and uniform crossover are considered in this study. Traditionally, GAs have used one and two-point crossover techniques, however more recent empirical studies have shown the benefits 
of using a higher number of crossover points [6]. Using uniform crossover should demonstrate the merit of a higher number of crossover points.

\subsubsection{Single-Point Crossover}

Single-point crossover is the most simplistic and most typically used crossover technique. The crossover point is determined randomly between 1 and the chromosome length. Once this point is reached, every gene from parent 1 is swapped with parent 2.

Random position $k=3$

Chromosome length $l=8$

\begin{tabular}{|c|c|c|c|c|c|c|c|c|c|}
\hline Parent 1 & 0.32 & 0.22 & 0.34 & 0.89 & 0.23 & 0.76 & 0.78 & 0.45 \\
\hline Parent 2 & 0.12 & 0.65 & 0.38 & 0.47 & 0.31 & 0.56 & 0.88 & 0.95 \\
\hline
\end{tabular}
$\begin{aligned} & \text { swapping all the } \\
& \text { genes between 4 } \\
& \text { and 8 }\end{aligned}$
\begin{tabular}{|c|c|c|c|c|c|c|c|c|}
\hline Offspring 1 & 0.32 & 0.22 & 0.34 & 0.47 & 0.31 & 0.56 & 0.88 & 0.95 \\
\hline Ofíspring 2 & 0.12 & 0.65 & 0.38 & 0.89 & 0.23 & 0.76 & 0.78 & 0.45 \\
\hline
\end{tabular}

Figure 2 - Single-Point Crossover [7]

\subsubsection{Two-Point Crossover}

The two-point crossover technique functions in the same way as single-point crossover in that genes from one parent are swapped with another at a particular, randomly selected point. The difference between the two being that there is one additional crossover point at which genes are swapped. In general, the same idea is applied to crossover techniques that employ a higher number of crossover points. 
Random position $k=3$

Random position $s=6$

\begin{tabular}{|l|l|l|l|l|l|l|l|l|}
\hline Parent 1 & 0.32 & 0.22 & 0.34 & 0.89 & 0.23 & 0.76 & 0.78 & 0.45 \\
\hline Parent 2 & 0.12 & 0.65 & 0.38 & 0.47 & 0.31 & 0.56 & 0.88 & 0.95 \\
\hline
\end{tabular}
$\begin{aligned} & \text { swapping all the } \\
& \text { genes between 4 } \\
& \text { and 6 }\end{aligned}$
\begin{tabular}{|c|c|c|c|c|c|c|c|c|}
\hline Ofíspring 1 & 0.32 & 0.22 & 0.34 & 0.47 & 0.31 & 0.56 & 0.78 & 0.45 \\
\hline Ofispring 2 & 0.12 & 0.65 & 0.38 & 0.89 & 0.23 & 0.76 & 0.88 & 0.95 \\
\hline
\end{tabular}

Figure 3 - Two-Point Crossover [7]

\subsubsection{Uniform Crossover}

The uniform crossover technique assigns genes from parent 1 to offspring 1 and parent 2 to offspring 2 with a probability of $p_{c}$ and vice versa [7]. This probability is typically $p_{c}=0.5$. To elaborate, as the technique iterates over the genes of each parent chromosome, a coin is flipped. If the coin were to land on heads, for example, the gene in question from parent 1 would be assigned to offspring 1 and the gene in question from parent two would be assigned to offspring 2. If the coin were to land on tails, the gene from parent 1 would be assigned to offspring 2 and the gene from parent 2 would be assigned to offspring 1 .

\begin{tabular}{|}
\begin{tabular}{|c|c|c|c|c|c|c|c|c|}
\hline Parent 1 & 0.32 & 0.22 & 0.34 & 0.89 & 0.23 & 0.76 & 0.78 & 0.45 \\
\hline Parent 2 & 0.12 & 0.65 & 0.38 & 0.47 & 0.31 & 0.56 & 0.88 & 0.95 \\
\hline $\begin{array}{c}\text { Random } \\
\text { Number }\end{array}$ & 0.32 & 0.22 & 0.34 & 0.89 & 0.23 & 0.76 & 0.78 & 0.45 \\
\hline Prob. Cross = 0.7 & $<0.7$ & $<0.7$ & $<0.7$ & $>0.7$ & $<0.7$ & $>0.7$ & $>0.7$ & $<0.7$ \\
\hline Offspring 1 & 0.32 & 0.22 & 0.34 & 0.89 & 0.23 & 0.76 & 0.78 & 0.45 \\
\hline Offspring 2 & 0.12 & 0.65 & 0.38 & 0.47 & 0.31 & 0.56 & 0.88 & 0.95 \\
\hline
\end{tabular}
\end{tabular}

Figure 4 - Uniform Crossover [7] 


\subsubsection{Mutation and Rate}

A mutation can be considered as a change in a chromosome to introduce a new individual to the

population. How the chromosome is changed varies based on how the problem is encoded. Like crossover, the probability of a mutation occurring can be represented as $p_{m}$ and is usually very low. The higher the mutation rate the more random the GA becomes. Mutation is an effective tool for maintaining and introducing diversity to a population.

\subsubsection{Maintaining Genetic Diversity}

Maintaining genetic diversity is the most important factor to reduce premature convergence, however forcing genetic diversity too aggressively can result in behavior that is excessively random and doesn't converge to an acceptable solution in a reasonable amount of time. Because of this, the general convention is to maintain a low mutation rate. The higher the mutation rate the closer, the GA gets to becoming a random search.

There are a multitude of ways to balance genetic diversity as Gupta and Ghafir discuss [18], but two methods were not mentioned and can be directly used in conjunction with or as a replacement to the mutation operator.

\subsubsection{Triggered Hypermutation}

Triggered Hypermutation is a relatively adaptive mutation method that functions by identifying suboptimal convergence or unacceptable population fitness. When some condition is met that the method deems undesirable it triggers a spike in the mutation rate, forcing the GA away from suboptimal convergence, effectively refreshing the population. 


\subsubsection{Random Immigrants}

While not a mutation method, random immigration is still considered an effective way of maintaining diversity. Rather than mutating the parent chromosomes' offspring, a new randomly generated individual is introduced to the population. Random immigration can effectively replace more traditional means of mutation, but both methods have their merits.

\subsubsection{Exit Conditions}

Choosing an appropriate exit condition is very important when performing an empirical study. Understand that the GA will continue to execute until it is told to stop, not necessarily when it finds a solution. Allowing a GA to solve for specific goals rather than a single universal figure can make certain results more observable. For example, having a GA solve only until it finds a solution can yield better results regarding the fitness function, pre-solution. If the exit condition is set to a maximum fitness or number of generations, the observable results would likely not tell the same story as they could become saturated with post-solution results.

\subsection{Parameter Control}

Parameter control refers to starting evolutionary algorithm execution with initial parameter values and adjusting them as the execution progresses [8]. It is reserved for adaptive or dynamic EAs and therefore will not be considered for this study as the goal is to identify optimal parameters which are difficult to determine if they are constantly changing. The results of this study may prove to be beneficial to GAs that use parameter control. 


\subsection{Parameter Tuning}

Adjusting a GA to achieve better results while maintaining fixed parameter values during

execution is known as parameter tuning [8]. This study uses empirical analysis to determine how adjustments should be made. The GA can be thought of as a toolbox, where each parameter can be represented as a tool. The essence of solving a problem is choosing the right tool for the job and in some cases creating a tool. For example, you wouldn't hammer a nail with a screwdriver and you wouldn't use truncation selection on a small population. However, unlike traditional tools, the defined roles of different GA parameters are not as cut and dried, therefore it is harder to determine the correct tool for the job.

Jebari and Madiafi conducted a study that compared 6 selection methods against a variety of test functions in different problem domains. They discovered that results differed significantly from one problem to another and determined that choosing an adequate selection method was hard to do for problems when there is no posterior verification of results [4]. There is no singular parameter value for any particular type of parameter that works in all test cases and problem domains.

\subsection{Statement of the Problem}

In practice, GA parameters have traditionally been chosen by convention (high crossover rate, low mutation rate, etc)[19], however this study explores conventional and unconventional parameter values for the types of parameters discussed in section 1 . Conclusions will be drawn based on observation of results in conjunction with an understanding of how different parameters 
change the behavior of the GA. Solution quality and computation time will largely be taken into consideration. The optimal parameters that are identified may be of use in building adaptive genetic algorithms for similar problem domains. These parameters may also be of use to individuals who endeavor to find increasingly efficient logic circuit designs. Logic circuit design in this study was based on building circuits with a reduced number of logic gates primarily, but ignored gate delays for the most part.

\section{General Overview of Combinational Logic Circuits}

This study assumes a certain amount of prior knowledge concerning digital logic, specifically regarding the function of logic gates. Methods of designing logic circuits in sum-of-products (SOP) and product-of-sums (POS) form will be mentioned and referenced, but not discussed at length. It is important to keep in mind that this study is primarily focused on tuning a genetic algorithm to find the best parameters for this specific problem domain. Discovering efficient logic circuits for a given output bit sequence is more of a side effect than the end goal.

A combinational logic circuit's output value is determined solely by the values of its inputs [9]. It can be composed of any number of logic gates, cascading or not, and can be represented by a truth table. Seven logic gates are considered in this study including: AND, OR, NOT, NAND, NOR, XOR, and XNOR.

Typically, human designers use traditional gate minimization tools such as the Karnaugh Map and Quine-McCluskey method to solve logic circuits. The biggest issue with these methods is that they don't consider the NAND, NOR, XOR, and XNOR logic gates as they always produce

logic circuits in SOP and POS form. A brief description of each form can be found in section 2.1 below. 


\subsection{Sum-of-Products and Product-of-Sums}

Sum-of-Products and Product-of-Sums logic circuits use AND, OR, and NOT gates. They have 2 levels where the first level contains a series of AND gates for SOP circuits and OR gates for POS circuits. The second level contains an OR gate for SOP and an AND gate for POS. Figure 5 below shows an example of a SOP and POS circuit.

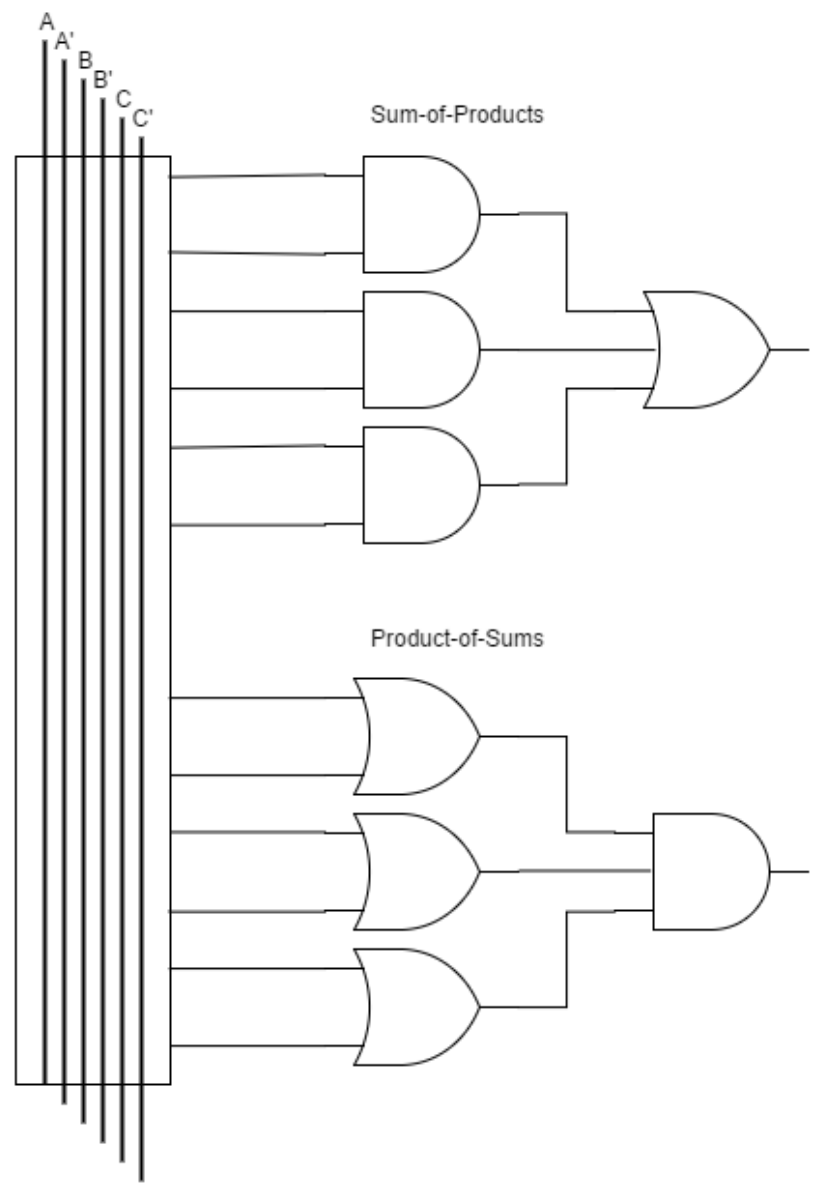

Figure 5 - SOP and POS 


\section{Why use a GA to Design Logic Circuits?}

It is easy enough to design a SOP or POS logic circuit. It can even be said that a SOP or POS circuit could then be optimized purely with universal gates like the NAND and NOR gate. However, as Rajaei, Houshmand, and Rouhani explain, optimizing a SOP/POS circuit is not the same as optimizing a circuit solved through evolutionary means [9]. There have been a handful

of other individuals and groups that have implemented GAs to design logic circuits in some way such as Coello, Christiansen, and Aguirre [10], Kalganova, Stomeo, Lambert, Lipnitsakya, and Yatskevitch [11], and Reis, Machado, and Cunha [12] just to name a few. There is a clear interest in this area of research which has become known as Evolutionary Electronics (EE) or Evolvable Hardware (EH) [12].

\subsection{Human Design Space vs. Evolutionary Computation Methods}

Miller, Job, and Vassilev [17] reference figure 6 in their study on the evolutionary design of digital circuits, which is a great representation in general, but especially so concerning logic circuit synthesis. SOP and POS circuits leave a lot to be desired as far as optimization is concerned. Human design is typically limited to the aforementioned gate minimization techniques in section 2 which are only able to use half of the available logic gates. Not only do evolutionary computation methods greatly expand the solution space, but they also offer the ability for humans to discern patterns from optimal solution sets that have the capacity to expand the human design space. 


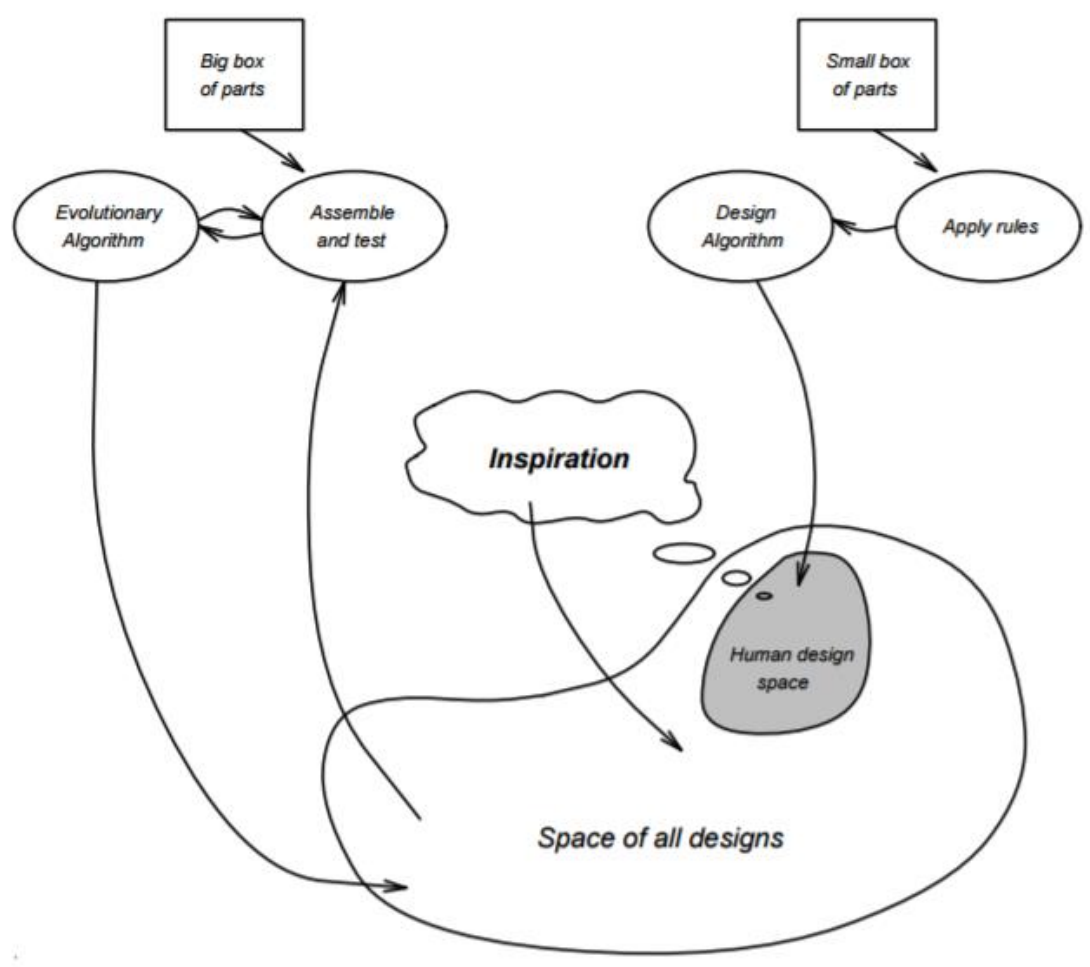

Figure 6 - Human Design Space vs. Evolutionary Design Space

\section{Encoding the Problem Domain}

Translating the problem in question to the software domain is the first real challenge when using a Genetic Algorithm. This concept is known as encoding. Compared to a more traditional encoding structure, like binary encoding, the structure used in this study is more complex, yet also more realistic and understandable. The core structures of the GA are represented as Plain Old Java Objects (POJOs).

\subsection{Logic Gate Representation}

Logic gates will essentially be the gene representation, but to reduce gene complexity the logic gate was given its own model. Logic gates are comprised of the following attributes: 
- gateId - An integer that is associated with a particular kind of gate. Ranges from 0-7:

$\circ \quad 0$ - Represents an AND gate

○ 1 - Represents an OR gate

○ 2 - Represents a NOT gate

○ 3 - Represents a NAND gate

○ 4 - Represents a NOR gate

○ 5 - Represents a XOR gate

○ 6 - Represents a XNOR gate

○ 7 - Represents a WIRE which is a non-operation and is also the default gate

- gateName - A name that is associated with each gate based on its gateId. It is used primarily for readability and is a secondary method of addressing a particular type of logic gate.

- inputs - A list of Boolean values that represent inputs to the gate.

- output - A Boolean value that represents a gate's output given a list of inputs.

\subsection{Gene Representation}

A gene is the most basic building block of the GA. Traditionally chromosomes are represented by sequences of genes in the context of the GA. Genes are comprised of the following attributes:

- gate - Represents a logic gate previously described in section 4.1.

- rowIndexInput 1 - Represents a row index for input wire 1.

- rowIndexInput2 - Represents a row index for input wire 2.

- solution - A Boolean value that represents whether the gene is part of a solution chromosome. 
- outputs - A list of all Boolean output values given a series of inputs.

\subsection{Chromosome Representation}

In this study, a chromosome is represented by a multi-dimensional array of genes previously described in section 4.2. Chromosomes are comprised of the following attributes:

- geneArray - Represents a multi-dimensional array filled with genes. The dimensions of the array are user-defined to be $n \times n$, where $n \geq$ the number of variables to be solved for.

- rouletteWeight - Represents a decimal value that denotes the percent chance the chromosome will be chosen for crossover.

- fitness - Represents the fitness of the chromosome.

- solutionLevel - Represents the level of the multi-dimensional array on which a solution was found for the chromosome. This value is used to map the solution backwards to accurately flag the logic gates that comprise the entire solution circuit.

- is Solution - A Boolean value that represents if the chromosome is a solution circuit.

\subsection{Population Representation}

The GA population is represented by a pool of chromosomes. The population size is userdefined. Populations are comprised of the following attributes:

- population - A list of chromosomes that represent the population and each chromosome represents an individual in the population.

- fitness - Represents the total fitness of the population.

- bestChromosome - Represents the chromosome with the highest fitness in the population. 
- solutionFound - A Boolean value that represents whether a solution chromosome has been found within the population.

\section{Fitness Function Disparity Between Solutions and Non-Solutions}

The fitness function is perhaps the single most important aspect of a Genetic Algorithm.

Whereas other traditional parameters are predefined tools, the fitness function must be designed to accommodate the problem domain. Because it is the only aspect of the GA that is based on user design, as much consideration was given to the fitness function as possible as it can greatly affect the performance of the GA. Due to the complexity of the fitness function, it remains static for all trials considered in this study.

The fitness function is split into two phases. The first phase focuses on awarding chromosomes before a solution circuit (chromosome) is found within the population. The second phase focuses on optimizing solution circuits after a solution has been discovered.

\subsection{Chromosome Fitness for Non-Solutions}

The first stage of the fitness function will judge a non-solution chromosome based on the highest number of correct outputs any particular gate in the chromosome produces. This number is stored in a variable maxCount and squared at the end of the function to get the fitness. Because of this, each additional correct output will give the chromosome a higher and higher fitness, making it more likely to be selected for crossover. Squaring maxCount isn't entirely necessary as maxCount alone will produce results. However, doing so places more reliance on the fitness function. 
To elaborate, a 3-variable function will have $2^{3}=8$ total output values, therefore the highest number of correct outputs a gate/gene could see before a solution is found is 7 . If a population

had one chromosome, A, with a maxCount of 3 and another chromosome, B, with a maxCount of 7, their fitness values would be 9 and 49 respectively. The total fitness of the population would be 58. This means that chromosome A would have a 7/58 (12\%) percent chance of being selected for crossover while chromosome B would have a 49/58 (84\%) percent chance of being selected using Roulette Wheel Selection. Compare this with the singular maxCount value: The total fitness of the population would be 10 . Chromosome A would have a 3/10 (30\%) percent chance of being selected while chromosome B would have a 7/10 (70\%) chance of being selected.

\subsection{Chromosome Fitness for Solutions}

The second stage of the function will judge a chromosome that has been deemed a solution based on a number of attributes. It is more complicated than stage one as there are many conditions that can adjust the chromosome's fitness. When a solution is detected the chromosome's fitness is spiked to bring it above non-solution chromosomes by a relatively significant amount. It should be noted that in order to compare the fitness of circuits of different dimensions, some method of normalizing the fitness must be developed. By this it is meant that a solution circuit found in a 4x4 matrix will have a much lower fitness value than that of a solution circuit found in a $5 \times 5$ matrix even if the $4 \times 4$ solution is better. Results analyzed in this study are only those obtained using the same solution space. 
- The first condition checked, after the fitness spike, is whether the chromosome's solution level is lower than the previous best chromosome in the population. If so increase the fitness.

Previous Chromosome Solution Level

\begin{tabular}{|c|c|c|c|c|c|}
\hline \multicolumn{2}{|r|}{ Level 1} & \multicolumn{2}{|r|}{ Level 2} & \multicolumn{2}{|r|}{ Level 3} \\
\hline WIRE [1 1] & 00110011 solution & WIRE [0 0] & 00110011 solution & AND [0 2] & 00010010 \\
\hline NAND [2 1] & 11111100 & NAND [1 1] & 00000011 & OR [1 2] & 01011011 \\
\hline XOR [2 0] & 01011010 solution & WIRE [2 2] & 01011010 solution & NAND [ 0 2] & 11101101 solution \\
\hline
\end{tabular}

Figure 7

Lower Solution Level Chromosome

\begin{tabular}{|c|c|c|c|}
\hline \multicolumn{2}{|r|}{ Level 1} & \multicolumn{2}{|r|}{ Level 2} \\
\hline WIRE [1 1] & 00110011 solution & AND [0 2] & 00010010 \\
\hline NAND [2 1] & 11111100 & OR [1 2] & 01011011 \\
\hline XOR [2 0] & 01011010 solution & NAND [ [ 2 2] & 11101101 solution \\
\hline
\end{tabular}

Figure 8

- In figure 7, the 3-variable solution circuit above, the initial solution chromosome had a solution level of 3 because that is the highest column number in which a logic gate was tagged as a solution.

○ In figure 8, a logic gate was tagged as a solution in a lower column than the previous solution, therefore this circuit would be awarded additional fitness points.

- The second condition checked determines how many gates/genes are NOT part of the solution. The higher the number, the better the solution. Fitness is increased based on how many non-solution gates/genes exist in the chromosome. 
- The third condition checked is broken into three parts:

If a WIRE is part of the solution

- Increase the fitness

If a NOT gate is part of the solution

- Increase the fitness

- If a NAND gate is part of the solution

- Increase the fitness

Results were obtained using the following fitness increments:

- Fitness spike: +2000 fitness

- Lower solution level: +100 fitness

- Non-solution gates: +50 fitness per gate

- WIRE: +50 fitness

- NOT: +25 fitness

- NAND: +10 fitness

\section{Analysis of Results}

Over 40 different combinations of GA parameters were tested to design combinational logic circuits. Most parameter sets were given 30 trials to accurately identify the behavior of the GA. Other parameter sets were given only 10-15 trials as their behavior was more apparent. The 
individual trial results and summary of trials for pre-solution and post-solution parameter sets can be found in the appendix.

\subsection{Benchmark Output Bit Sequence}

3-variable circuit design was too simple to use for this empirical study. Solutions were found too quickly and did not produce sufficiently meaningful results to accurately draw conclusions about different parameter sets.

5-variable circuit design tended to take exceedingly long to use for the purposes of research; however, if one had unlimited time, designing 5-variable circuits would likely reflect GA behavior better than the chosen 4-variable design.

The 4-variable output bit sequence chosen was one with no discernable pattern and could be considered as a more complex 4-variable output. Less complex 4-variable output bit sequences can have the same issue as 3-variable outputs in that solutions are found too quickly. An example of a simple 4-variable output compared to that of the chosen output is shown below.

Simple 4-variable output bit sequence:

1111000011110000

Chosen Benchmark 4-variable output bit sequence:

1110010010111010

Note that the simple 4-variable output has an easily discernable pattern in alternating groups of 4 bits. The benchmark output bit sequence has no discernable pattern and is naturally more complex. The design of combinational circuits for given output bit sequences are unique in their complexity, but these two examples are at opposite ends of the spectrum.

\subsection{Initial Solution Quality}


There is a very small discrepancy in fitness, for most parameter sets, regarding the initial solution found by the GA using a variety of parameters across all trials conducted. The range of average initial quality across all trial results, pre-solution and post-solution, but disregarding higher dimension solution spaces (see section 6.5 for reasoning and discussion), was 735 fitness. The low fitness being 2415 and the high fitness being 3150. However, the average initial solution quality of most parameter sets fell within 2600 and 3000 fitness. A fitness gap of 400 was too small to accurately draw conclusions about the affect different parameter sets could have upon initial solution quality. If the initial solution fitness gap was greater than 400 between two sets of parameters, it was noted.

In summary, no correlation was found between GA parameter set and the quality of the initial solution found.

\subsection{Fitness Ceiling}

Most trials performed for post-solution optimization were given 300 seconds to optimize initial solutions found. After identifying two parameter sets with high fitness gain for 300 seconds, parameter set D and C (parameter set $\mathrm{P}$ had a slightly higher average fitness gain, but used a different population size than parameter set D; see post-solution summary in appendix), they were each given 50 seconds and then 100 seconds to optimize. The fitness gain steadily increased for parameter sets S, R, D (all of which used RWS and single-point crossover) meaning a fitness ceiling was not hit with less than 300 seconds of optimization time allotted. The average fitness gain gap between parameter sets $\mathrm{V}, \mathrm{U}, \mathrm{C}$ (all of which used random tournament selection and single-point crossover) was much smaller than the aforementioned parameter sets. After this was discovered, the optimization time of parameter set D and C was 
increased to 1000 seconds (see parameter set $\mathrm{Q}$ and $\mathrm{T}$ in figure 9). The results using this optimization time were interesting; however, only 10 trials were performed for each parameter set. After 10 trials, for parameter set Q, the fitness gain was an average of 2086 which was less than the average fitness gain of the same parameter set, D, using a 300 second optimization time. This suggests that the time allotted to the GA to achieve the fitness ceiling on average wouldn't be much more or less than 300 seconds. However, an anomaly was discovered that blurs this conclusion (see section 6.4). In addition to this anomaly, 1000 seconds was also allotted to parameter set $\mathrm{C}$, the results of which also contradicted the previous conclusion drawn from parameter set $\mathrm{D}$ to $\mathrm{Q}$. The average fitness gain for parameter set $\mathrm{T}$ (parameter set $\mathrm{C}$ with 1000 seconds of optimization time) was 2446 which was a noticeable increase from that of parameter set C which only had an average fitness gain of 2019.

It should be noted that parameter set $\mathrm{C}$ and $\mathrm{T}$ use Tournament Selection with a tournament pool of size 1 (random selection). This may influence the gap between initial solution quality and the fitness ceiling. More about these findings are discussed in section 6.9.2. Further research may be required to accurately describe GA behavior concerning this concept. 


\begin{tabular}{|c|c|c|c|c|c|c|}
\hline Trial Set & Exit Condition & Population Selection Method & Solution Space (array dimensions) & Crossover Technique & Crossover Rate Mutation Technique & Mutation Rate \\
\hline A & 300 seconds & 100 RWS & $4 \times 4$ & Single-point & 0.8 Random gene replacement & 0.2 \\
\hline B & 300 seconds & 100 RWS & $4 \times 4$ & Uniform (rate $=0.5$ ) & 0.8 Random gene replacement & 0.2 \\
\hline C & 300 seconds & $100 \mathrm{TS}(1)$ & $4 \times 4$ & Single-point & 0.8 Random gene replacement & 0.2 \\
\hline D & 300 seconds & 100 RWS & $4 \times 4$ & Single-point & 0.8 Random gene replacement & 0.2 \\
\hline $\mathrm{E}$ & 300 seconds & $100 \mathrm{TS}(20)$ & $4 \times 4$ & Single-point & 0.8 Random gene replacement & 0.2 \\
\hline $\mathrm{F}$ & 300 seconds & $100 \mathrm{TS}(50)$ & $4 \times 4$ & Single-point & 0.8 Random gene replacement & 0.2 \\
\hline G & 300 seconds & $100 \mathrm{TS}(5)$ & $4 \times 4$ & Single-point & 0.8 Random gene replacement & 0.2 \\
\hline $\mathrm{H}$ & 300 seconds & $100 \mathrm{TS}(20)$ & $4 \times 4$ & Uniform (rate $=0.5$ ) & 0.8 Random gene replacement & 0.2 \\
\hline I & 300 seconds & $100 \mathrm{TS}(50)$ & $4 \times 4$ & Uniform (rate $=0.5$ ) & 0.8 Random gene replacement & 0.2 \\
\hline $\mathrm{J}$ & 300 seconds & $100 \mathrm{TS}(5)$ & $4 \times 4$ & Uniform (rate $=0.5$ ) & 0.8 Random gene replacement & 0.2 \\
\hline $\mathrm{K}$ & 300 seconds & 500 RWS & $4 \times 4$ & Uniform (rate $=0.5$ ) & 0.8 Random gene replacement & 0.2 \\
\hline L & 300 seconds & 50 RWS & $4 \times 4$ & Uniform (rate $=0.5$ ) & 0.8 Random gene replacement & 0.2 \\
\hline $\mathrm{M}$ & 1382 seconds & 500 RWS & $4 \times 4$ & Uniform (rate $=0.5$ ) & 0.8 Random gene replacement & 0.2 \\
\hline $\mathrm{N}$ & 290 seconds & 50 RWS & $4 \times 4$ & Uniform (rate $=0.5$ ) & 0.8 Random gene replacement & 0.2 \\
\hline 0 & 300 seconds & 500 RWS & $4 \times 4$ & Single-point & 0.8 Random gene replacement & 0.2 \\
\hline $\mathrm{P}$ & 300 seconds & $500 \mathrm{TS}(1)$ & $4 \times 4$ & Single-point & 0.8 Random gene replacement & 0.2 \\
\hline Q & 1000 seconds & 100 RWS & $4 \times 4$ & Single-point & 0.8 Random gene replacement & 0.2 \\
\hline $\mathrm{R}$ & 100 seconds & 100 RWS & $4 \times 4$ & Single-point & 0.8 Random gene replacement & 0.2 \\
\hline $\mathrm{S}$ & 50 seconds & 100 RWS & $4 \times 4$ & Single-point & 0.8 Random gene replacement & 0.2 \\
\hline$T$ & 1000 seconds & $100 \mathrm{TS}(1)$ & $4 \times 4$ & Single-point & 0.8 Random gene replacement & 0.2 \\
\hline u & 100 seconds & $100 \mathrm{TS}(1)$ & $4 \times 4$ & Single-point & 0.8 Random gene replacement & 0.2 \\
\hline V & 50 seconds & $100 \mathrm{TS}(1)$ & $4 \times 4$ & Single-point & 0.8 Random gene replacement & 0.2 \\
\hline W & 300 seconds & 100 RWS & $4 \times 4$ & Single-point & 0.5 Random gene replacement & 0.5 \\
\hline $\mathrm{x}$ & 1258.5 seconds & $500 \mathrm{TS}(1)$ & $4 \times 4$ & Single-point & 0.8 Random gene replacement & 0.2 \\
\hline $\mathrm{Y}$ & 156.12 seconds & $50 \mathrm{TS}(1)$ & $4 \times 4$ & Single-point & 0.8 Random gene replacement & 0.2 \\
\hline Z & 300 seconds & $100 \mathrm{TS}(1)$ & $4 \times 4$ & Uniform (rate $=0.5$ ) & 0.8 Random gene replacement & 0.2 \\
\hline AA & 300 seconds & $100 \mathrm{TS}(1)$ & $4 \times 4$ & Uniform (rate $=0.5$ ) & 0.5 Random gene replacement & 0.5 \\
\hline BB & 1381.2 seconds & 500 RWS & $4 \times 4$ & Single-point & 0.8 Random gene replacement & 0.2 \\
\hline
\end{tabular}

Figure 9 - Post-Solution Parameter Sets

\subsection{Anomaly Concerning Fitness Ceiling}

A second set of 30 trials were performed on parameter set D (parameter set A). Interestingly the results were significantly less ideal in parameter set $\mathrm{A}$. In parameter set $\mathrm{D}$, the average fitness gain was 2375.3, compared to the average fitness gain of parameter set A which was 1688.4.

This is a difference of 686.9 fitness which cannot be ignored. In addition to this, parameter set D had an average initial solution quality of 2837.3 which was lower than that of parameter set A which had an average initial solution quality of 2998.6. These results could be due to several factors: 
1) More trials may need to be conducted per set possibly, although the difference in average fitness gain between these two identical parameter sets was rather large for this to be the case.

2) Based on the genetic makeup (logic gate makeup) of each chromosome initially discovered to be a solution, there is only so much room to grow. If the discrepancy between unique chromosome fitness ceilings is too high, results can be skewed in an unpredictable manner.

\subsection{Solution Space Analysis}

After analyzing 30 trials for a set of non-ideal parameters (parameter set $\mathrm{P}$ from the pre-solution summary; see appendix or figure 10) and identifying a premature convergence (PC) rate of 30\%, the dimensions of the solution space matrix were increased from $4 \times 4$ to $6 \times 6$. The PC rate dropped to $0 \%$, but the issue with this increase in solution space is that a way to normalize the fitness between solutions using different dimension matrices was never found.

A larger solution space means more overall genes (logic gates) to consider. Typically, there are more gates that are and are not part of the solution. Because the fitness function awards chromosomes (circuits) created based on their composition, having more genes (logic gates) means more opportunity to be awarded fitness. Due to this, only a $4 \times 4$ solution space was considered in this study. 


\subsection{Population Size Analysis}

\subsubsection{Pre-Solution}

For the best parameter set pre-solution (parameter set B; see pre-solution summary appendix),

the population was altered to compare computation times and premature convergence (PC) rates.

Parameter set K was the same as parameter set B except with a population size of 500 instead of

100. The PC rate was $0 \%$ and had an average computation time of 40.475 seconds.

Parameter set L was the same as parameter set B except with a population size of 50 instead of

100. The PC rate was also $0 \%$ and had an average computation time of 8.896 seconds.

Parameter set $\mathrm{B}$ had a population size of 100 . The PC rate was $0 \%$ and the average computation time was 5.937 seconds. This shows that the behavior of the GA favors smaller populations presolution as it performed much better with population sizes of 50 and 100 rather than 500 .

\begin{tabular}{|c|c|c|c|c|c|c|c|}
\hline Trial Set & Exit Condition & Population Selection Method & Solution Space & Crossover Technique & Crossover Rate & Mutation Technique & Mutation Rate \\
\hline A & Solution Stop & 100 RWS & $4 \times 4$ & Single-point & 0.8 & Random Gene Mutation & 0.2 \\
\hline B & Solution Stop & 100 RWS & $4 \times 4$ & Uniform (rate $=0.5$ ) & 0.8 & Random Gene Mutation & 0.2 \\
\hline C & Solution Stop & $100 \mathrm{TS}(1)$ & $4 \times 4$ & Single-point & 0.8 & Random Gene Mutation & 0.2 \\
\hline D & Solution Stop & $100 \mathrm{TS}(1)$ & $4 \times 4$ & Uniform (rate $=0.5$ ) & 0.8 & Random Gene Mutation & 0.2 \\
\hline$E$ & Solution Stop & $100 \mathrm{TS}(20)$ & $4 \times 4$ & Single-point & 0.8 & Random Gene Mutation & 0.2 \\
\hline $\mathrm{F}$ & Solution Stop & $100 \mathrm{TS}(50)$ & $4 \times 4$ & Single-point & 0.8 & Random Gene Mutation & 0.2 \\
\hline G & Solution Stop & $100 \mathrm{TS}(5)$ & $4 \times 4$ & Single-point & 0.8 & Random Gene Mutation & 0.2 \\
\hline $\mathrm{H}$ & Solution Stop & $100 \mathrm{TS}(20)$ & $4 \times 4$ & Uniform (rate $=0.5$ ) & 0.8 & Random Gene Mutation & 0.2 \\
\hline I & Solution Stop & $100 \mathrm{TS}(50)$ & $4 \times 4$ & Uniform (rate $=0.5$ ) & 0.8 & 3 Random Gene Mutation & 0.2 \\
\hline J & Solution Stop & $100 \mathrm{TS}(5)$ & $4 \times 4$ & Uniform (rate $=0.5$ ) & 0.8 & Random Gene Mutation & 0.2 \\
\hline K & Solution Stop & 500 RWS & $4 \times 4$ & Uniform (rate $=0.5$ ) & 0.8 & Random Gene Mutation & 0.2 \\
\hline L & Solution Stop & 50 RWS & $4 \times 4$ & Uniform (rate $=0.5$ ) & 0.8 & Random Gene Mutation & 0.2 \\
\hline M & Solution Stop & 100 RWS & $6 \times 6$ & Uniform (rate $=0.5)$ & 0.8 & Random Gene Mutation & 0.2 \\
\hline $\mathrm{N}$ & Solution Stop & $100 \mathrm{TS}(20)$ & $6 \times 6$ & Single-point & 0.8 & Random Gene Mutation & 0.2 \\
\hline 0 & Solution Stop & 100 RWS & $4 \times 4$ & Uniform (rate $=0.5$ ) & 0.5 & Random Gene Mutation & 0.5 \\
\hline $\mathrm{P}$ & Solution Stop & $100 \mathrm{TS}(20)$ & $4 \times 4$ & Single-point & 0.5 & Random Gene Mutation & 0.5 \\
\hline
\end{tabular}

Figure 10 - Pre-Solution Parameter Sets 


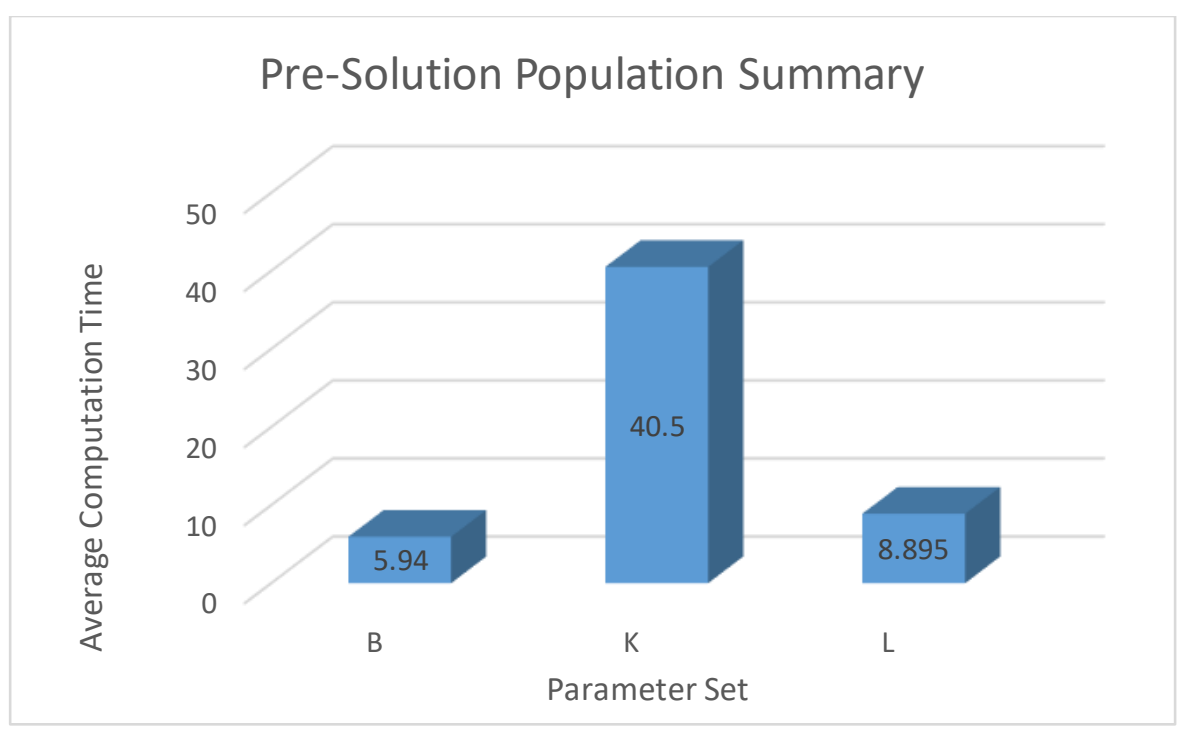

Figure 11 - Pre-solution population analysis

\subsubsection{Post-Solution}

To begin the post-solution population analysis, the same parameter set was used as in the presolution analysis. However, the result of interest for post-solution is average fitness gain rather than computation time. Interestingly, there is not a noticeable difference in fitness gain for any parameter sets with different populations while other parameters are held constant. No conclusions could be drawn about population size. It seems population size is a more important factor pre-solution than post-solution. 


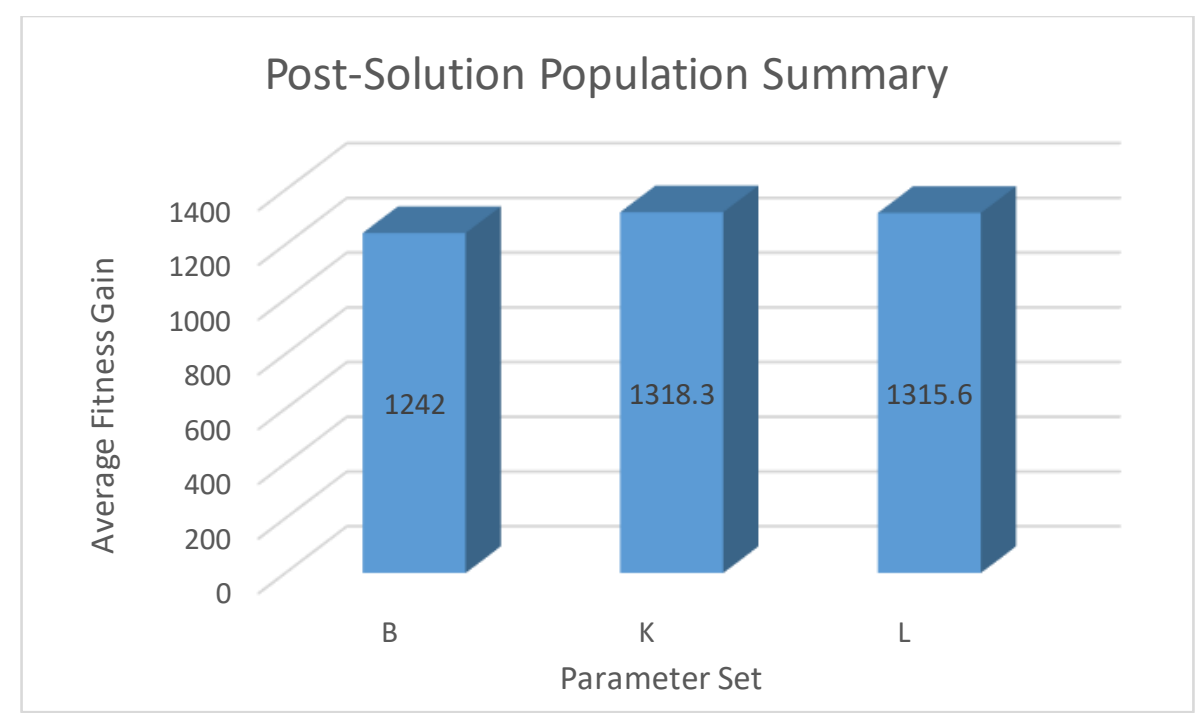

Figure 12 - Post-solution population analysis

\subsection{Crossover and Mutation Rate Analysis}

Conventionally, the crossover rate has been held relatively high, around $80 \%$ and the mutation rate held relatively low, around $20 \%$. Mutation is only considered if crossover has already occurred in this study. Conventional and unconventional crossover and mutation rates are considered in sections 6.7.1 and 6.7.2 below.

\subsubsection{Pre-Solution}

The best and worst parameter sets pre-solution were examined using conventional and unconventional crossover and mutation rates. Parameter set B using the conventional 0.8 crossover rate and 0.2 mutation rate produced an average computation time of 5.9 seconds. The same parameter set, $\mathrm{O}$, using the unconventional 0.5 crossover rate and 0.5 mutation rate, produced an average computation time of 48.2 seconds. 
Parameter set $\mathrm{E}$ using conventional crossover and mutation rates produced an average computation time of 112 seconds and had a premature convergence rate of $60 \%$. The same parameter set, $\mathrm{P}$, using the unconventional crossover and mutation rates produced an average computation time 24.7 seconds and had a premature convergence rate of $30 \%$.

Not surprisingly, conventional crossover and mutation rates caused greater performance than that of unconventional rates for good parameter sets, but the unconventional rates caused greater performance for bad parameter sets. This gives credence to the triggered hypermutation method discussed in section 1.3.6.1.1.

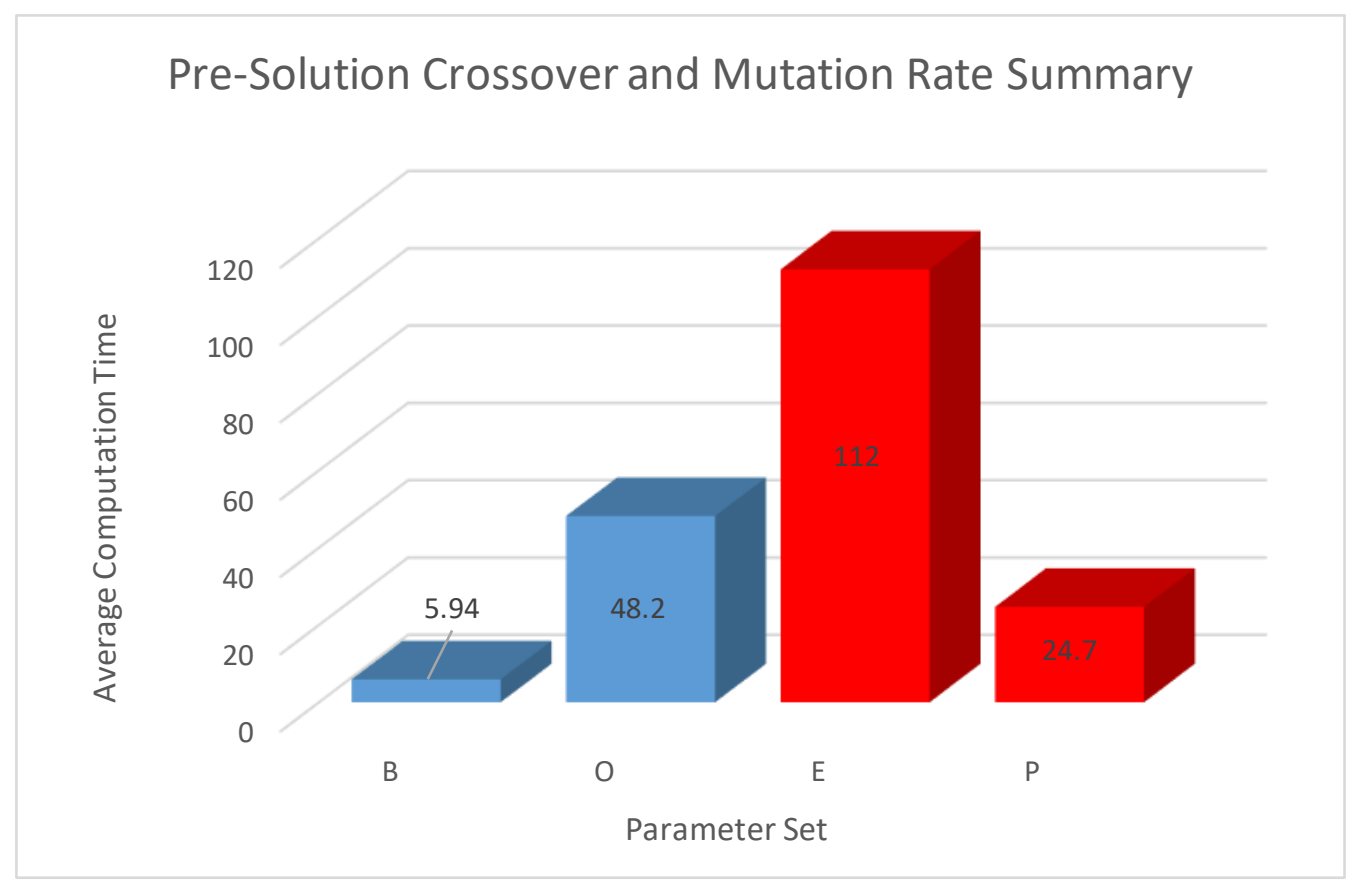

Figure 13 - Pre-solution crossover and mutation rate analysis

\subsubsection{Post-Solution}

Post-solution results were not as clear due to the anomaly discussed in section 6.4 as the result of changing the crossover and mutation rates of parameter set $\mathrm{D}$ to 0.5 resulted in an average fitness 
gain lower than the initial average, but higher than the anomaly. This result can be seen in the post-solution summary, parameter set $\mathrm{E}$, in the appendix.

Parameter set $\mathrm{Z}$ resulted in an average fitness gain of 2236.7 using conventional crossover and mutation rates. Parameter set AA used identical parameters as Z, but used unconventional crossover and mutation rates which resulted in a very similar average fitness gain of 2288.7 .

It was surprising to see there was no clear advantage to using conventional vs. unconventional crossover and mutation rates, especially considering post-solution results use the phase 2 fitness function which more accurately awards chromosomes.

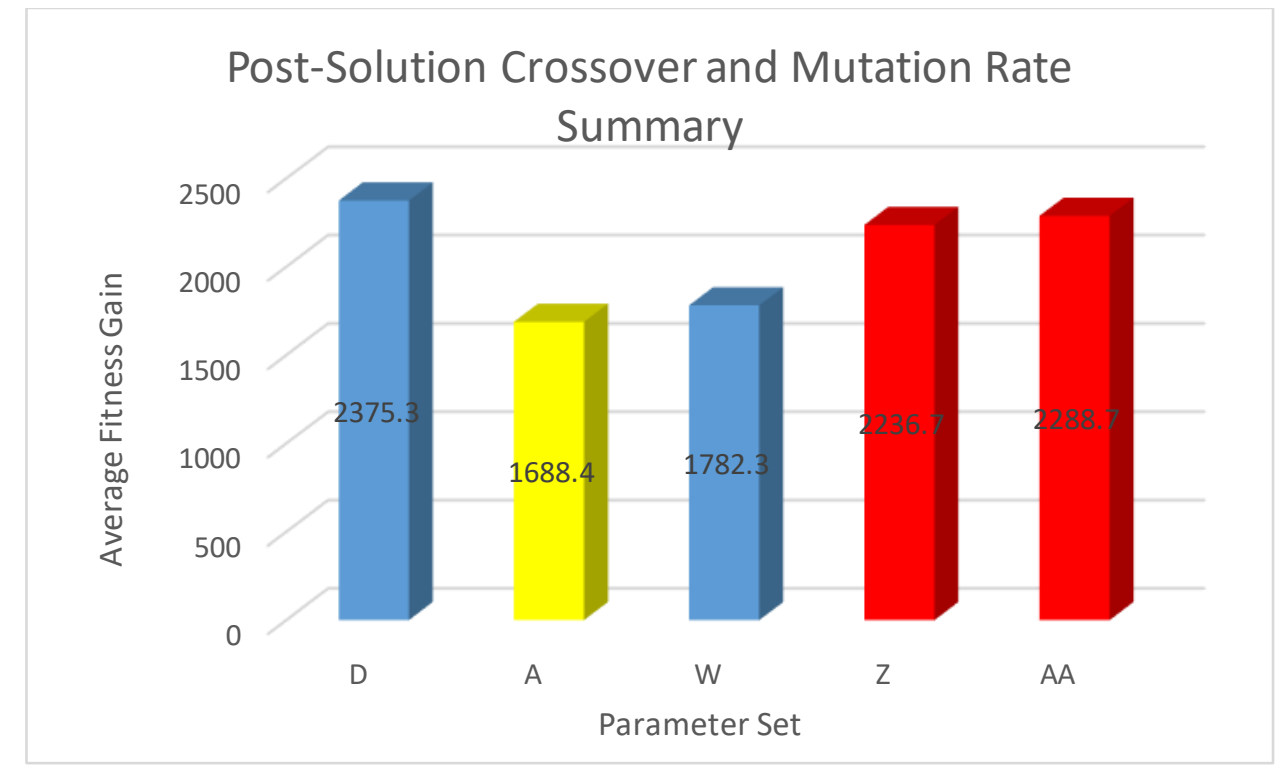

Figure 14 - Post-solution crossover and mutation rate analysis

\subsection{Crossover Technique Analysis}

Crossover techniques were an interesting parameter to study. As Hasançebi and Erabatur explore in their evaluation of crossover techniques [16], there is an ongoing debate about the merits of techniques based on the number of crossover points, yet their chosen problem to solve 
had a somewhat less defined line regarding solution vs. non-solution. Logic circuit synthesis is a good problem domain to study GA parameters more closely, allowing exploration of candidate chromosomes pre-solution and post-solution where the fitness function is weak and strong respectively. Because the primary debate concerning crossover techniques focuses on the number of crossover points, two techniques were explored, single-point crossover and uniform crossover with a 0.5 rate. Uniform crossover was chosen as the primary solution space was a $4 \times 4$ matrix for 4 -variable circuits which equates to a 16 -space gene sequence. This means there is a possible 16 crossover points, but on average 8 crossover points with a 0.5 rate.

\subsubsection{Pre-Solution}

The selection methods used in conjunction with each crossover technique affected premature convergence rates to some extent; however, any parameter set that used RWS and uniform crossover had a premature convergence rate of $0 \%$. See figure 10 for pre-solution parameter sets.

Parameter set B, which used RWS and uniform crossover, produced the best average computation time at 5.9 seconds and had a $0 \%$ PC rate, while parameter set A, which used RWS and single-point crossover, had an average computation time of 53.7 seconds and a PC rate of $10 \%$. While holding all other parameters constant other than crossover technique and seeing a significantly lower computation time and a lower $\mathrm{PC}$ rate from parameter set $\mathrm{B}$, it can be concluded that uniform crossover is the stronger crossover technique pre-solution. This may also suggest that a higher number of crossover points can aide a GA that lacks a strong fitness function. 


\subsubsection{Post-Solution}

The post-solution crossover technique analysis produced some very interesting results. It was assumed that after finding uniform crossover to be the ideal crossover technique pre-solution, it would also be the ideal technique post-solution, yet this was not the case. Single-point crossover results trumped nearly all uniform crossover results (see post-solution summary in appendix).

\subsection{Selection Method Analysis}

2 selection methods were focused on for this study, Roulette Wheel Selection (RWS) and Tournament Selection. Many other selection methods use RWS as the basis of their function, therefore RWS was the base selection method focused on in this study.

\subsubsection{Pre-Solution}

The pre-solution results clearly establish that RWS is the superior selection method concerning this problem domain. See section 6.8.1. The differences in computation time between RWS and tournament selection were not excessively large, but were usually weighted in favor of RWS. On top of this, RWS most certainly performed better regarding premature convergence rate. See post-solution summary in the appendix, section 9.3.1.

\subsubsection{Post-Solution}

The post-solution selection method results were perhaps the most interesting yet controversial and unexpected results obtained in this study. Tournament Selection using tournaments of 5, 20, and 50 produced the worst results regardless of crossover technique, failing to even break an average fitness gain of 1000 . It was very surprising to see this was the case as larger tournament 
pools place a higher reliance on the fitness function and phase 2 of the fitness function was much stronger than phase 1.

The best pre-solution parameter set, B, also had bad performance with an average fitness gain of 1242. In general, when RWS was used in conjunction with uniform crossover, post-solution, results were consistently sub-optimal.

It appears that RWS when used in conjunction with single-point crossover produced results that were initially considered ideal; however, after allotting more optimization time, it was discovered that RWS hit a lower fitness ceiling on average than Tournament Selection using tournaments of size $1(\mathrm{TS}(1))$.

With an optimization time of 300 seconds, the results were very similar as can be seen between parameter set $\mathrm{C}$ and $\mathrm{D}$ (taking the anomaly, $\mathrm{A}$, into account), or $\mathrm{O}$ and $\mathrm{P}$.

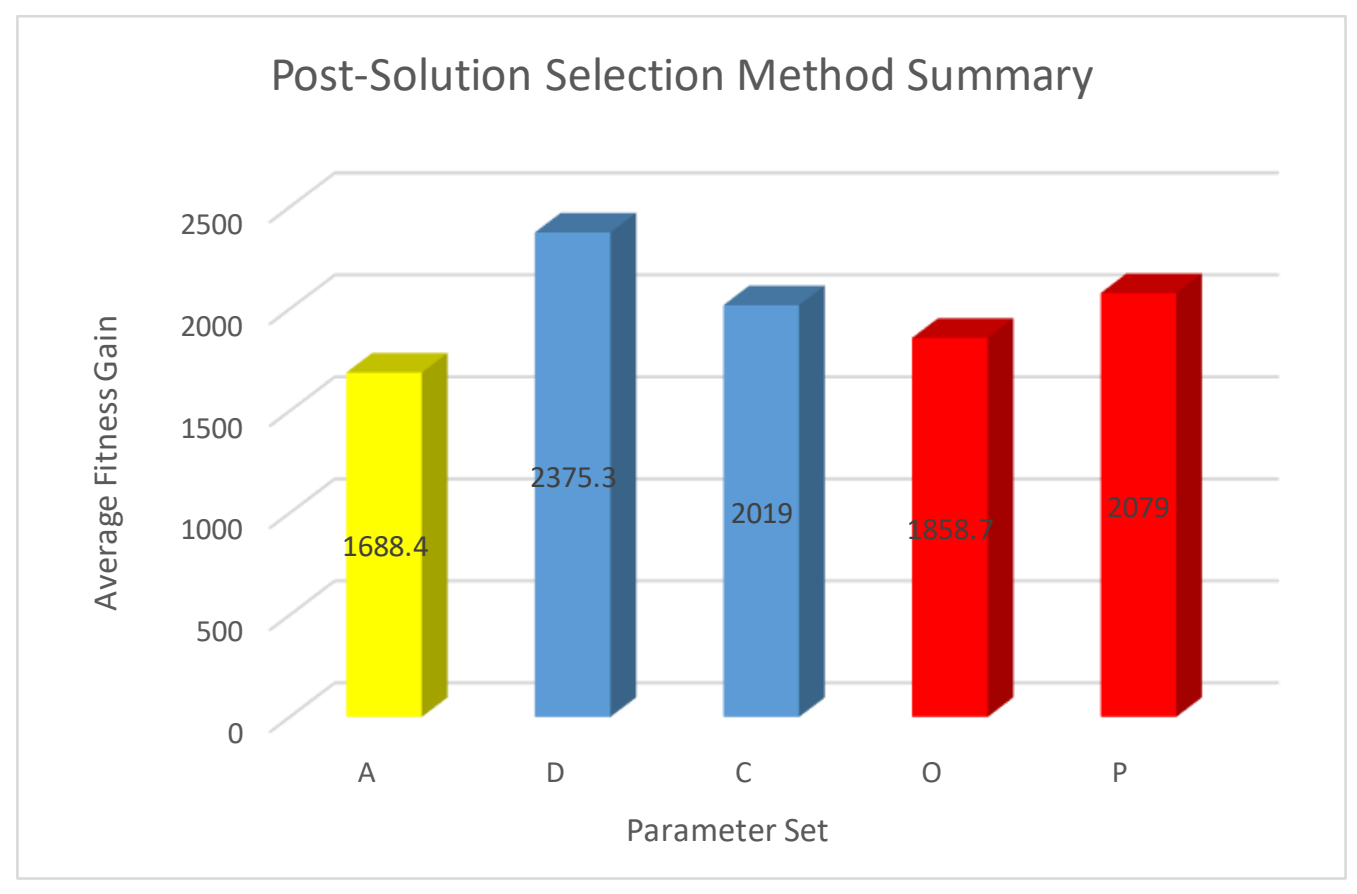

Figure 15 - Post-solution selection method analysis (300 seconds) 
However, when RWS and TS(1) were allotted 1000+ seconds of optimization time, a gap in results began to appear between the two selection methods. Parameter set Q, which used RWS and single-point crossover, produced an average fitness gain of 2086 while parameter set T, which used TS(1) and single-point crossover, produced an average fitness gain of 2446 . To further explore whether this gap was just another anomaly, RWS and TS(1) were both given 30,000 generations to optimize and their populations were increased to 500 each. This equated to an average computation time of 1381.2 seconds for the RWS parameter set, BB, and an average computation time of 1258.5 seconds for the TS(1) parameter set, X. Parameter set BB produced an average fitness gain of 2069 while parameter set X produced an average fitness gain of 2940.7. It was concluded that TS(1) was consistently improving the fitness ceiling past a point where RWS could not.

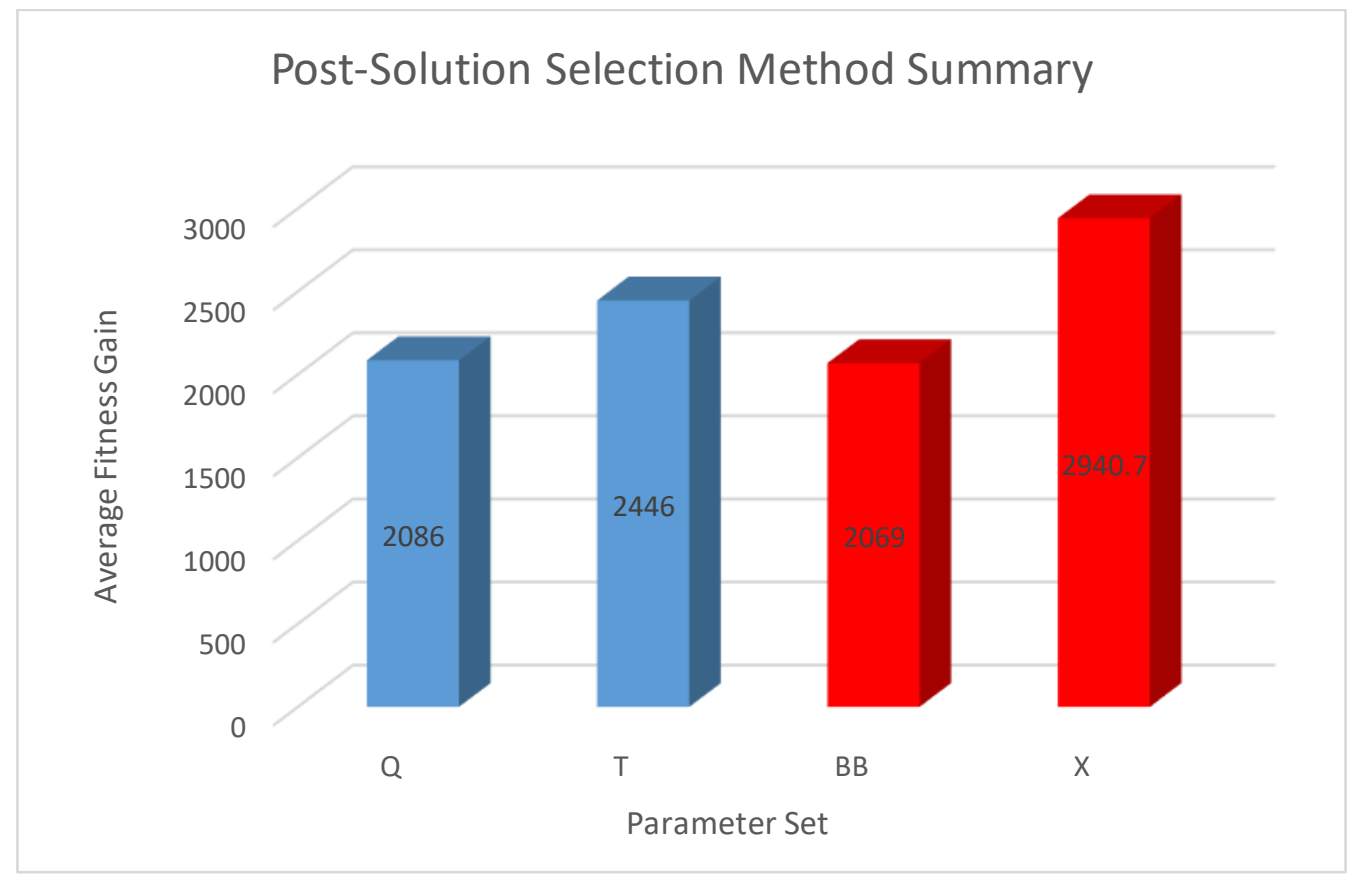

Figure 16 - Post-solution selection method analysis (1000+ seconds) 
These findings introduce a concept that could be specific to the synthesis of logic circuits. To find a circuit with a higher fitness ceiling, it appears that maintaining as much genetic diversity as possible trumps anything the fitness function can do for the GA. This concept was further explored by adjusting other parameters used in conjunction with TS(1). Unconventional crossover and mutation rates were used as well as uniform crossover, parameter sets AA and Z respectively, neither of which had an adverse effect on the results. In fact, both parameter sets produced an average fitness gain of over 2200.

In summary, random selection, TS(1), was able to surpass local optima more efficiently than other selection methods and get closer to a global optimum. This was very interesting to discover and shows that each problem domain has its own unique complexities concerning GA parameter tuning.

\section{Conclusion}

Parameter tuning a GA used to design combinational logic circuits produced some unique results. Letting the GA operate until an initial solution was found allowed the analysis of parameters pre-solution, while letting the GA operate for a set amount of time or generations allowed the analysis of parameters post-solution. Pre-solution and post-solution results are found in the following sections. The results of this study may be useful for building adaptive GAs for this problem domain or for individuals who endeavor to design increasingly efficient logic circuits. Circuits designed in this study are efficient regarding the number of logic gates used, but do not consider gate delays. 


\subsection{Pre-Solution}

Consolidating all subsections of section 6, the ideal parameters, of those tested, used to achieve an initial solution circuit were Roulette Wheel Selection (RWS), uniform crossover, smaller population sizes (specifically a size of 50-100 produced the best pre-solution results), random gene mutation, a crossover rate of 0.8 and a mutation rate of 0.2 . This parameter set produced an average computation time of 5.94 seconds and a $0 \%$ premature convergence rate. Noting that the discrepancy in fitness between non-solution circuits was very small allowed the evaluation of parameters without the significant influence of the fitness function.

\subsection{Post-Solution}

The post-solution results were more complex as it was unexpected that tournament selection using tournaments of size 1 (TS(1)) would perform so well. Parameter sets that used RWS and single-point crossover kept pace with sets that used TS(1) until a local optimum or "fitness ceiling” was hit. Once RWS and single-point crossover sets hit their fitness ceiling, TS(1) parameter sets began to outshine them. See post-solution summary in appendix, section 9.3.1.

Post-solution trials used a stronger fitness function, increasing the discrepancy in fitness between circuits, which in turn increased the discrepancy in selection weight. Because random selection (TS(1)) ultimately performed better than RWS, it appears that maintaining genetic diversity trumps a good fitness function for this problem.

TS(1) was not significantly impacted by other parameter values, considering post-solution results. The parameter set that produced the best results, did so because it was given 
significantly longer than other parameter sets that used TS(1). This was done to compare the fitness ceilings of the best sets using RWS to that of TS(1), holding other parameters constant. Uniform crossover and unconventional crossover and mutation rates did not have an adverse effect on TS(1) as it did with RWS for post-solution optimization.

\subsection{Future Considerations}

For future consideration and work, automating the data collection process would allow for a much more thorough investigation. Time saved using automated data collection could have been put towards building a program that allows for greater exploration of the problem domain.

Giving logic gates (genes) the ability to have more than 2 inputs is something I would have liked to accomplish as results could be more comparable to human design where results are typically in POS and SOP form.

Employing more selection methods and crossover techniques or even building my own, is something I would love to tackle as well. I would also like to take some of my findings and step into the realm of adaptive GAs. 


\section{References}

[1] Melanie, M. (1999). An Introduction to Genetic Algorithms. Cambridge, MA: A Bradford Book, The MIT Press

[2] Roeva, O., Fidanova, S., \& Paprzycki, M. (2013). Influence of the Population Size on the Genetic Algorithm Performance in Case of Cultivation Process Modelling. Proceedings of the 2013 Federated Conference on Computer Science and Information Systems. pp. 371-376

[3] Baker, J. E. (1987). Reducing Bias and Inefficiency in the Selection Algorithm. Proceedings of the Second International Conference on Genetic Algorithms. pp. 14-21

[4] Jebari, K. (2014). Selection Methods for Genetic Algorithms. Retrieved from https://www.researchgate.net/publication/259461147_Selection_Methods_for_Genetic_Algorith $\mathrm{ms}$

[5] Obitko, M. (1998). Introduction to Genetic Algorithms. Retrieved from http://www.obitko.com/tutorials/genetic-algorithms/selection.php

[6] Spears, V. M., \& De Jong, K. A. (1991). On the Virtues of Parameterized Uniform Crossover. Proceedings of the Fourth International Conference on Genetic Algorithms. pp. 230-236

[7] Magalhães-Mendes, J. (2013). A Comparative Study of Crossover Operators for Genetic Algorithms to Solve the Job Shop Scheduling Problem. WSEAS Transactions on Computers. Issue 4, Vol. 12

[8] Eiben, A. E., Hinterding, R., \& Michalewicz, Z. (1999). Parameter Control in Evolutionary Algorithms. IEEE Transactions on Evolutionary Computation. Vol. 3, No. 2

[9] Rajaei, A., Houshmand, M., \& Rouhani, M. (2011). Optimization of Combinational Logic Circuits Using NAND Gates and Genetic Programming. Retrieved from https://www.researchgate.net/publication/225644919_Optimization_of_Combinational_Logic_C ircuits_Using_NAND_Gates_and_Genetic_Programming

[10] Coello, C. A., Christiansen, A. D., \& Aguirre, A. H. (1997). Automated Design of Combinational Logic Circuits by Genetic Algorithms. Proceedings of the International Conference in Norwich, U.K. pp. 333-336

[11] Stomeo, E., Kalganova, T., Lambert, C., Lipnitsakya, N., \& Yatskevich, Y. (2005). On Evolution of Relatively Large Combinational Logic Circuits. Proceedings of the 2005 NASA/DoD Conference of Evolution Hardware.

[12] Baker, J. E. (1985). Adaptive Selection Methods for Genetic Algorithms. Proceedings of the First International Conference on Genetic Algorithms and Their Applications. pp. 101-135 
[13] Andrea, J., Siarry, P., \& Dognon, T. (2001). An Improvement of the Standard Genetic Algorithm Fighting Premature Convergence in Continuous Optimization. Advances in Engineering Software. Vol. 32, Issue 1. pp. 49-60

[14] Holland, J. H. (1992). Adaptation in Natural and Artificial Systems. Cambridge, MA: The MIT Press

[15] Shukla, A., Pandey, H. M., \& Mehrotra, D. (2015). Comparative Review of Selection Techniques in Genetic Algorithm. IEEE International Conference on Futuristic Trends in Computational Analysis and Knowledge Management.

[16] Hasançebi, O., \& Erbatur, F. (1998). Evaluation of Crossover Techniques in Genetic Algorithm Based Optimum Structural Design. Computers and Structures.

[17] Miller, J. F., Job, D., \& Vassilev, V. K. (1999). Principles in the Evolutionary Design of Digital Circuits - Part 1. Genetic Programming and Evolvable Machines.

[18] Gupta, D., \& Ghafir, S. (2012). An Overview of Methods Maintaining Diversity in Genetic Algorithms. International Journal of Emerging Technology and Advanced Engineering. Vol. 2, Issue 5

[19] Eiben, A. E., \& Smit, S. K. (2012). Chapter 2 - Evolutionary Algorithm Parameters and Methods to Tune Them. Autonomous Search. pp. 308

[20] Miller, B. L., \& Goldberg, D. E. (1995). Genetic Algorithms, Tournament Selection, and the Effects of Noise. Complex Systems. Vol 9, pp. 193-212 


\section{Appendix}

\subsection{Description of Program}

The GA begins with an initial population of randomly generated chromosomes. Each

chromosome represents an $n \times n$ matrix filled with genes, where $n$ is the number of variables.

Each gene represents a logic gate object and the 2 inputs and output of each respective logic gate.

The number of inputs was decided to be 2 for simplicity and as it has seen success by Coello, Christiansen, and Aguirre [10]. Each gate must be connected to its respective inputs. Once the circuit is connected, it has become functional and it must be determined whether the candidate chromosome is a solution. This is done by plugging in a generated truth table to the circuit and scanning the entire circuit for a solution bit sequence. If a candidate chromosome is found to be a solution it is flagged as such and each gene that is part of the solution is also flagged. Once each chromosome is scanned, the fitness function evaluates each chromosome and assigns each a fitness value. The fitness value of every chromosome in the population is summed. Each chromosome's fitness is then divided by the fitness of the population to obtain its selection weight which is used in the process of selecting chromosomes for crossover and mutation. Only 2 chromosomes are selected for crossover and produce 2 offspring. If crossover does not occur (this study used crossover rates of 0.8 and 0.5 ), a new chromosome was generated to replicate random immigration as explained in section 1.3.6.1.2. It should be noted that once a solution is found, the GA will never lose a solution. The best chromosome is always carried into the population. This is known as elitism, specifically having 1 elite chromosome. This process is repeated for $x$ generations where each new population is considered a single generation. 


\subsection{Pre-Solution Trial Results}

\subsubsection{Pre-Solution Summary}

\begin{tabular}{|l|l|l|l|}
\hline Trial Set & Exit Condition & Population Selection Method & Solution Space \\
\hline A & Solution Stop & 100 RWS & $4 \times 4$ \\
\hline B & Solution Stop & 100 RWS & $4 \times 4$ \\
\hline C & Solution Stop & 100 TS(1) & $4 \times 4$ \\
\hline D & Solution Stop & 100 TS(1) & $4 \times 4$ \\
\hline E & Solution Stop & 100 TS(20) & $4 \times 4$ \\
\hline F & Solution Stop & 100 TS(50) & $4 \times 4$ \\
\hline G & Solution Stop & 100 TS(5) & $4 \times 4$ \\
\hline H & Solution Stop & 100 TS(20) & $4 \times 4$ \\
\hline I & Solution Stop & 100 TS(50) & $4 \times 4$ \\
\hline J & Solution Stop & 100 TS(5) & $4 \times 4$ \\
\hline K & Solution Stop & 500 RWS & $4 \times 4$ \\
\hline L & Solution Stop & 50 RWS & $4 \times 4$ \\
\hline M & Solution Stop & 100 RWS & $6 \times 6$ \\
\hline N & Solution Stop & 100 TS(20) & $6 \times 6$ \\
\hline O & Solution Stop & 100 RWS & $4 \times 4$ \\
\hline P & Solution Stop & 100 TS(20) & $4 \times 4$ \\
\hline & & & \\
\hline
\end{tabular}

\begin{tabular}{|l|r|r|r|}
\hline Crossover Technique & Crossover Rate & Mutation Technique & Mutation Rate \\
\hline Single-point & 0.8 Random Gene Mutation & 0.2 \\
\hline Uniform (rate $=0.5)$ & 0.8 Random Gene Mutation & 0.2 \\
\hline Single-point & 0.8 Random Gene Mutation & 0.2 \\
\hline Uniform (rate $=0.5)$ & 0.8 Random Gene Mutation & 0.2 \\
\hline Single-point & 0.8 Random Gene Mutation & 0.2 \\
\hline Single-point & 0.8 Random Gene Mutation & 0.2 \\
\hline Single-point & 0.8 Random Gene Mutation & 0.2 \\
\hline Uniform (rate $=0.5)$ & 0.8 Random Gene Mutation & 0.2 \\
\hline Uniform (rate $=0.5)$ & 0.8 Random Gene Mutation & 0.2 \\
\hline Uniform (rate $=0.5)$ & 0.8 Random Gene Mutation & 0.2 \\
\hline Uniform (rate $=0.5)$ & 0.8 Random Gene Mutation & 0.2 \\
\hline Uniform (rate $=0.5)$ & 0.8 Random Gene Mutation & 0.2 \\
\hline Uniform (rate $=0.5)$ & 0.8 Random Gene Mutation & 0.2 \\
\hline Single-point & 0.8 Random Gene Mutation & 0.2 \\
\hline Uniform (rate $=0.5)$ & 0.5 Random Gene Mutation & 0.5 \\
\hline Single-point & 0.5 Random Gene Mutation & 0.5
\end{tabular}

\begin{tabular}{|c|c|c|c|c|}
\hline Trial Set & Average Initial Solution Quality & Average Computation Time (seconds) & Average Generations & Premature Convergence Rate ( 30 Trials) \\
\hline A & 2660.4 & 53.7 & 6218.8 & $10 \%$ \\
\hline B & 2868.7 & 5.94 & 570.3 & $0 \%$ \\
\hline C & 2843.6 & 43.6 & 5298.4 & $6.70 \%$ \\
\hline D & 2715.8 & 26.4 & 3020.2 & $13.30 \%$ \\
\hline $\mathrm{E}$ & 2667.5 & 112 & 13602.5 & $60 \%$ (10 trials) \\
\hline $\mathrm{F}$ & 2988.6 & 22.9 & 2686.7 & $30 \%$ (10 trials) \\
\hline G & 2623.3 & 71.8 & 9008.5 & $40 \%$ (10 trials) \\
\hline $\mathrm{H}$ & 2631.3 & 18.9 & 2137.6 & $20 \%$ (10 trials) \\
\hline I & 2768 & 44.3 & 5144.6 & $50 \%$ (10 trials) \\
\hline J & 2778 & 73.7 & 8912 & $50 \%$ (10 trials) \\
\hline K & 2960.3 & 40.5 & 1081.4 & $0 \%$ \\
\hline L & 2730.3 & 8.895 & 1002.6 & $0 \%$ \\
\hline M & 4693 & 10.2 & 466.9 & $0 \%$ \\
\hline $\mathrm{N}$ & 5470.3 & 32.7 & 1645 & $0 \%$ \\
\hline $\mathrm{O}$ & 2794.6 & 48.2 & 5105.6 & $0 \%$ \\
\hline P & 2837.1 & 24.7 & 2489.7 & $30 \%$ \\
\hline
\end{tabular}

\section{Pre-Solution Summary}

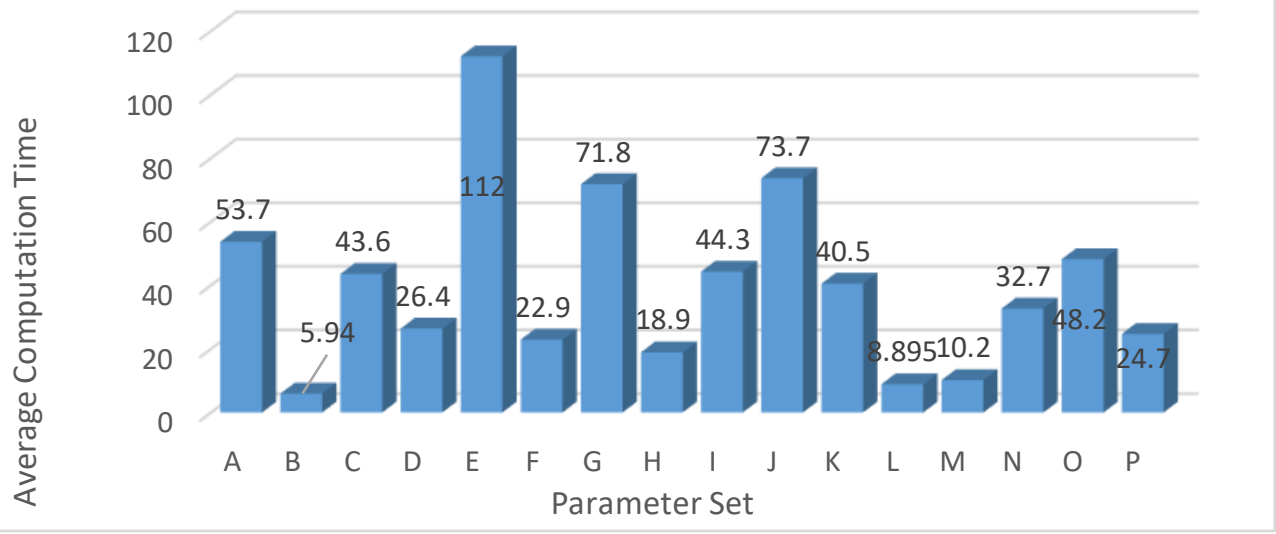




\subsubsection{Pre-Solution Trials}
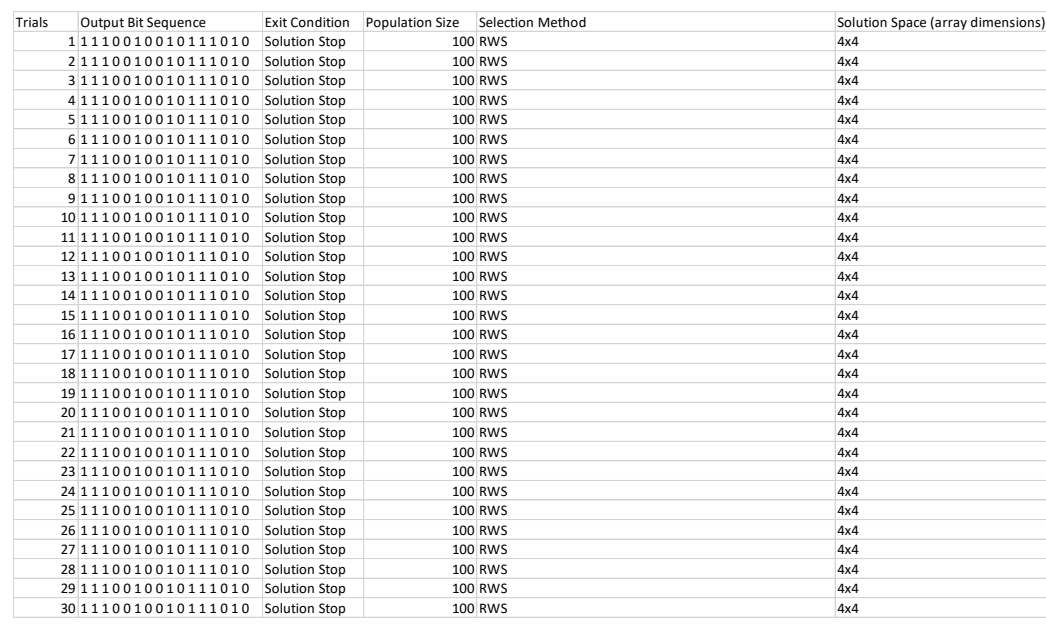

Crossover Technique Crossover Rate Mutation Technique

single-point 0.8 Random Gene Replacement

0.8 Random Gene Replacement

$\begin{array}{ll} & 0.8 \text { Random Gene Replacement } \\ \text { single-point } & 0.8 \text { Random Gene Replacement }\end{array}$

single-point 0.8 Random Gene Replacement

Single-point 0.8 Random Gene Replacement

Single-point $\quad 0.8$ Random Gene Replacement

$\begin{array}{ll}\text { Single-point } & 0.8 \text { Random Gene Replacement } \\ \text { Single-point } & 0.8 \text { Random Gene Replacement }\end{array}$

Single-point 0.8 Random Gene Replacement

$\begin{array}{ll}\text { Single-point } & 0.8 \text { Random Gene Replacement } \\ \text { single-point } & 0.8 \text { Random Gene Replacement }\end{array}$

Single-point $\quad 0.8$ Random Gene Replacement

Single-point 0.8 Random Gene Replacement

Single-point 0.8 Random Gene Replacement

Single-point $\quad 0.8$ Random Gene Replacement

Single-point $\quad 0.8$ Random Gene Replacement

Single-point 0.8 Random Gene Replacement

$\begin{array}{ll}\text { Single-point } & 0.8 \text { Random Gene Replacement } \\ \text { Single-point } & 0.8 \text { Random Gene Replacement }\end{array}$

$\begin{array}{ll}\text { Single-point } & 0.8 \text { Random Gene Replacement } \\ \text { Single-point } & 0.8 \text { Random Gene Replacement }\end{array}$

$\begin{array}{ll}\text { single-point } & 0.8 \text { Random Gene Replacement } \\ \text { Single-point } & 0.8 \text { Random Gene Replacement }\end{array}$

Single-point 0.8 Random Gene Replacement

single-point

Single-point 0.8 Random Gene Replacement

Single-point

0.8 Random Gene Replacement

\begin{tabular}{ll} 
Single-point & 0.8 Random Gene Replacement \\
\hline Single-point & 0.8 Random Gene Replacement
\end{tabular}

Mutation Rate

\begin{tabular}{|r|r|r|}
\hline Computation Time (seconds) & Generations & Solution Quality (fitness) \\
\hline 2.597 & 178 & 2300
\end{tabular}

$1.714 \quad 103 \quad 2620$

\begin{tabular}{r|r|r|}
1.696 & 92 & 3370
\end{tabular}

\begin{tabular}{r|r|r|}
1.582 & 37 & 2460 \\
\hline 7.488 & 740
\end{tabular}

\begin{tabular}{|r|r|r|}
\hline 7.488 & 740 & 3570
\end{tabular}

$24.326 \quad 2787 \quad 2410$

\begin{tabular}{|c|c|c|c|}
\hline N/A & N/A & N/A & No solution after 50,000 generations \\
\hline 14.958 & 1678 & 2350 & \\
\hline 15.861 & 1780 & 2450 & \\
\hline 3.158 & 275 & 2460 & \\
\hline 395.403 & 46898 & 2970 & \\
\hline 1.267 & 45 & 2300 & \\
\hline 2.234 & 148 & 2410 & \\
\hline 3.233 & 272 & 2460 & \\
\hline 200.426 & 22573 & 2460 & \\
\hline 14.893 & 1655 & 2570 & \\
\hline 1.327 & 70 & 3370 & \\
\hline 5.807 & 560 & 2450 & \\
\hline 3.236 & 273 & 2410 & \\
\hline 15.435 & 1780 & 2730 & \\
\hline 186.869 & 22552 & 2450 & \\
\hline 405.743 & 50047 & 3050 & \\
\hline 82.155 & 9689 & 2250 & \\
\hline N/A & N/A & $\mathrm{N} / \mathrm{A}$ & No solution after 50,000 generations \\
\hline 9.779 & 1100 & 2860 & \\
\hline 3.365 & 296 & 2450 & \\
\hline 1.785 & 120 & 2610 & \\
\hline N/A & $\mathrm{N} / \mathrm{A}$ & $\mathrm{N} / \mathrm{A}$ & No solution after 50,000 generations \\
\hline 5.907 & 303 & 3180 & \\
\hline 37.09 & 1856 & 2860 & \\
\hline 53.67903704 & 6218.777778 & 2660.37037 & \\
\hline
\end{tabular}



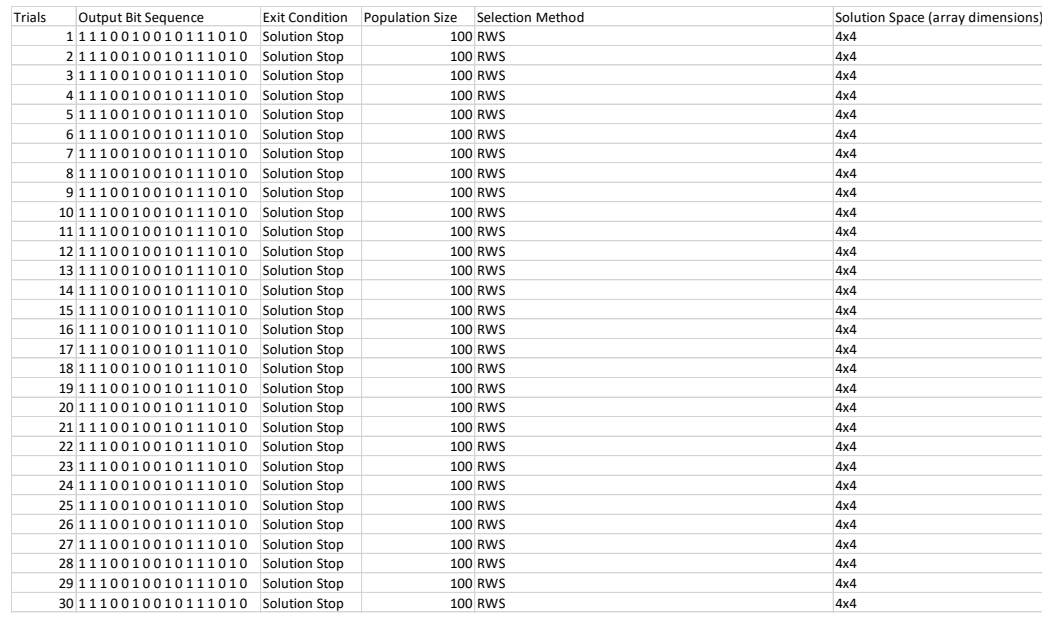

Crossover Technique Crossover Rate Mutation Technique 0.8 Random Gene Replacement 0.8 Random Gene Replacement Uniform (rate $=0.5) \quad 0.8$ Random Gene Replacement Uniform (rate $=0.5$ ) 0.8 Random Gene Replacement Uniform (rate $=0.5$ ) $\quad 0.8$ Random Gene Replacement Uniform (rate $=0.5$ ) $\quad 0.8$ Random Gene Replacement Uniform (rate $=0.5$ ) $\quad 0.8$ Random Gene Replacement Uniform (rate $=0.5$ ) $\quad 0.8$ Random Gene Replacement Uniform (rate $=0.5) \quad 0.8$ Random Gene Replacement Uniform (rate $=0.5) \quad 0.8$ Random Gene Replacement Uniform $($ rate $=0.5) \quad 0.8$ Random Gene Replacement Uniform (rate $=0.5) \quad 0.8$ Random Gene Replacement Uniform (rate $=0.5$ ) $\quad 0.8$ Random Gene Replacement Uniform (rate $=0.5$ ) $\quad 0.8$ Random Gene Replacement Uniform (rate $=0.5$ ) $\quad 0.8$ Random Gene Replacement Uniform (rate $=0.5$ ) $\quad 0.8$ Random Gene Replacement Uniform (rate $=0.5$ ) $\quad 0.8$ Random Gene Replacement Uniform (rate $=0.5) \quad 0.8$ Random Gene Replacement Uniform (rate $=0.5) \quad 0.8$ Random Gene Replacement Uniform (rate $=0.5) \quad 0.8$ Random Gene Replacement Uniform $($ rate $=0.5) \quad 0.8$ Random Gene Replocement Uniform (rate $=0.5$ ) $\quad 0.8$ Random Gene Replacement Uniform (rate $=0.5$ ) $\quad 0.8$ Random Gene Replacement Uniform (rate $=0.5$ ) $\quad 0.8$ Random Gene Replacement 0.8 Random Gene Replacement $\begin{array}{ll}\text { Uniform }(\text { rate }=0.5) & 0.8 \text { Random Gene Replacement } \\ \text { Uniform (rate }=0.5) & 0.8 \text { Random Gene Replacement }\end{array}$ Uniform (rate $=0.5)$

\begin{tabular}{|c|c|c|}
\hline Computation Time (seconds) & Generations & Solution Quality (fitness) \\
\hline 2.378 & 209 & 2700 \\
\hline 2.683 & 119 & 2700 \\
\hline 1.778 & 110 & 2300 \\
\hline 1.84 & 126 & 3100 \\
\hline 2.765 & 221 & 2810 \\
\hline 10.734 & 1176 & 2460 \\
\hline 2.183 & 160 & 2700 \\
\hline 1.18 & 48 & 2460 \\
\hline 7.866 & 835 & 3260 \\
\hline 3.664 & 341 & 2300 \\
\hline 2.783 & 225 & 3260 \\
\hline 9.384 & 1026 & 2460 \\
\hline 2.001 & 147 & 2450 \\
\hline 3.25 & 285 & 2810 \\
\hline 3.044 & 264 & 2460 \\
\hline 11.772 & 1318 & 2810 \\
\hline 10.138 & 440 & 2810 \\
\hline 5.404 & 543 & 3660 \\
\hline 18.638 & 2151 & 2610 \\
\hline 4.27 & 385 & 2610 \\
\hline 7.58 & 467 & 2460 \\
\hline 1.808 & 124 & 2700 \\
\hline 24.341 & 2690 & 3260 \\
\hline 3.281 & 291 & 3420 \\
\hline 3.838 & 357 & 3770 \\
\hline 3.737 & 327 & 3420 \\
\hline 9.196 & 1024 & 2620 \\
\hline 2.9 & 251 & 3370 \\
\hline 7.423 & 796 & 3210 \\
\hline 6.263 & 654 & 3100 \\
\hline 5.9374 & 570.3333333 & 2868.666667 \\
\hline
\end{tabular}




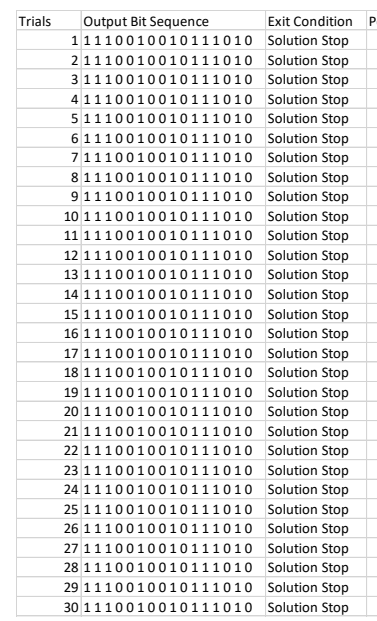

Selection Method

00 Tournament Selection - Size $=1$ (random selection) Size $=1$ (random selection) ( Delection - Size $=1$ (random selection) 100 Tournament Selection - Size $=1$ (random selection) 00 Tournament Selection - Size $=1$ (random selection) 100 Tournament Selection - Size $=1$ (random selection) 100 Tournament Selection - Size $=1$ (random selection) Toumament Selection-Size $=1$ (random selection) To Tournament Selection - Size $=1$ (random selection) DO Tournament Selection - Size $=1$ (random selection) Too Tournament Selection - Size $=1$ ( 100 Tournament Selection - Size $=1$ (random selection) 100 Tournament Selection - Size $=1$ (random selection) 100 Tournament Selection - Size $=1$ (random selection) 100 Tournament Selection - Size $=1$ (random selection) 100 Tournament Selection - Size $=1$ (random selection) 100 Tournament Selection - Size $=1$ (random selection) 100 Toumant selection - Size $=1$ (random selection) To Tomament Selection - Size $=1$ (random selection) 100 Tournament Selection - Size $=1$ (random selection) 100 Tournament Selection - Size $=1$ (random selection) 100 Tournament Selection - Size $=1$ (random selectio 100 Tournament Selection - Size $=1$ (random selectio $)$ 100 Tournament Selection - Size $=1$ (random selectio $)$

\begin{tabular}{l} 
Solution Space (array dimensions) \\
\hline $4 \times 4$ \\
$4 \times 4$ \\
$4 \times 4$ \\
$4 \times 4$ \\
$4 \times 4$ \\
$4 \times 4$ \\
$4 \times 4$ \\
$4 \times 4$ \\
$4 \times 4$ \\
$4 \times 4$ \\
$4 \times 4$ \\
\hline $4 \times 4$ \\
$4 \times 4$ \\
$4 \times 4$ \\
$4 \times 4$ \\
$4 \times 4$ \\
$4 \times 4$ \\
$4 \times 4$ \\
$4 \times 4$ \\
$4 \times 4$ \\
$4 \times 4$ \\
$4 \times 4$ \\
$4 \times 4$ \\
$4 \times 4$ \\
$4 \times 4$ \\
$4 \times 4$ \\
\hline $4 \times 4$ \\
$4 \times 4$ \\
$4 \times 4$ \\
$4 \times 4$ \\
\hline
\end{tabular}

Crossover Technique Crossover Rate Mutation Technique 0.8 Random Gene Replacement $\begin{array}{ll}0.8 \text { Random Gene Replacement } \\ \text { Single-point } & 0.8 \text { Random Gene Replacement }\end{array}$ $\begin{array}{ll}\text { Single-point } & 0.8 \text { Random Gene Replacement } \\ \text { Sing } & 0.8 \text { Random Gene Replacement }\end{array}$ Single-point $\quad 0.8$ Random Gene Replacement Single-point $\quad 0.8$ Random Gene Replacement Single-point 0.8 Random Gene Replacement Single-point 0.8 Random Gene Replacement $\begin{array}{ll}\text { Single-point } & 0.8 \text { Random Gene Replacement } \\ \text { Single-point } & 0.8 \text { Random Gene Replacement }\end{array}$ $\begin{array}{ll}\text { Single-point } & 0.8 \text { Random Gene Replacement } \\ \text { Single-point } & 0.8 \text { Random Gene Replacement }\end{array}$ $\begin{array}{ll} & 0.8 \text { Random Gene Replacement } \\ \text { single-point } & 0.8 \text { Random Gene Replacement } \\ \text { Single-point } & 0.8 \text { rond }\end{array}$ Single-point 0.8 Random Gene Replacement single-point 0.8 Random Gene Replacement Single-point $\quad 0.8$ Random Gene Replacement single-point 0.8 Random Gene Replacement Single-point 0.8 Random Gene Replacement Single-point 0.8 Random Gene Replacement single-point 0.8 Random Gene Replacement Single-point 0.8 Random Gene Replacement $\begin{array}{ll}\text { Single-point } & 0.8 \text { Random Gene Replacement } \\ \text { Single-point } & 0.8 \text { Random Gene Replacement }\end{array}$ Single-point 08 Random Gene Replocement Single-point 0.8 Random Gene Replacement Single-point 0.8 Random Gene Replacement single-point $\quad 0.8$ Random Gene Replacement 0.8 Random Gene Replacement single-point $\quad 0.8$ Random Gene Replacement 0.8 Random Gene Replacement

Mutation Rate single-poin

Computation Time (seconds) Generations Solution Quality (fitness)

\begin{tabular}{|c|c|c|c|}
\hline 248.113 & 30214 & 2460 & \\
\hline 1.225 & 53 & 3410 & \\
\hline N/A & N/A & N/A & No solution after 50,000 generations \\
\hline 23.031 & 2728 & 2810 & \\
\hline 1.013 & 39 & 3010 & \\
\hline 55.735 & 6774 & 3050 & \\
\hline 52.177 & 6240 & 3100 & \\
\hline 1.59 & 80 & 2850 & \\
\hline 34.408 & 4185 & 2810 & \\
\hline 3.424 & 290 & 3260 & \\
\hline 47.634 & 5814 & 2300 & \\
\hline 1.92 & 131 & 3530 & \\
\hline 6.155 & 652 & 3770 & \\
\hline 250.292 & 31566 & 3260 & \\
\hline 2.459 & 183 & 2460 & \\
\hline 1.614 & 91 & 2300 & \\
\hline 65.293 & 8015 & 3010 & \\
\hline 1.476 & 79 & 3100 & \\
\hline 19.807 & 2306 & 2300 & \\
\hline 0.971 & 38 & 3260 & \\
\hline 2.412 & 186 & 2300 & \\
\hline 5.898 & 578 & 3900 & \\
\hline 24.074 & 2832 & 2620 & \\
\hline 9.192 & 1030 & 2300 & \\
\hline 339.21 & 41987 & 2610 & \\
\hline N/A & N/A & N/A & No solution after 50,000 generations \\
\hline 13.572 & 1502 & 2460 & \\
\hline 5.659 & 566 & 2780 & \\
\hline 1.111 & 45 & 2300 & \\
\hline 2.141 & 152 & 2300 & \\
\hline 43.62878571 & 5298.428571 & 2843.571429 & \\
\hline
\end{tabular}



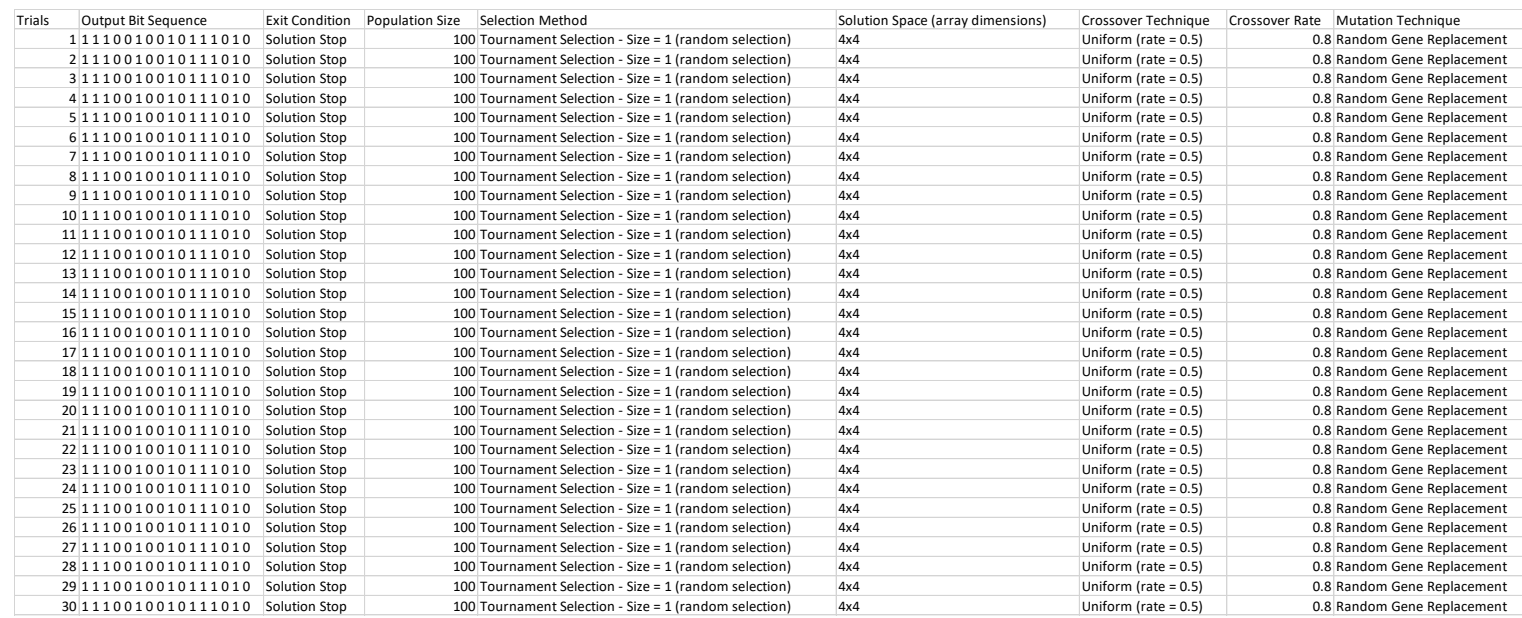

\begin{tabular}{|r|}
\hline Mutation Rate \\
0.2 \\
0.2 \\
0.2 \\
0.2 \\
0.2 \\
0.2 \\
0.2 \\
0.2 \\
0.2 \\
0.2 \\
0.2 \\
0.2 \\
0.2 \\
0.2 \\
0.2 \\
0.2 \\
0.2 \\
0.2 \\
0.2 \\
0.2 \\
0.2 \\
0.2 \\
0.2 \\
0.2 \\
0.2 \\
0.2 \\
0.2 \\
0.2 \\
0.2 \\
0.2 \\
\hline
\end{tabular}

\begin{tabular}{|c|c|c|}
\hline Computation Time (seconds) & Generations & Solution Quality (fitness) \\
\hline N/A & N/A & $\mathrm{N} / \mathrm{A}$ \\
\hline 15.8 & 1820 & 2450 \\
\hline 37.664 & 4541 & 2250 \\
\hline 34.217 & 4093 & 3020 \\
\hline 1.042 & 46 & 2450 \\
\hline 2.245 & 182 & 2300 \\
\hline 3.15 & 272 & 2860 \\
\hline 43.201 & 1806 & 2300 \\
\hline 3.277 & 290 & 2460 \\
\hline 1.09 & 46 & 3100 \\
\hline 1.222 & 43 & 3260 \\
\hline 5.599 & 442 & 3770 \\
\hline N/A & N/A & $N / A$ \\
\hline 1.219 & 56 & 2300 \\
\hline 4.301 & 408 & 3100 \\
\hline 104.59 & 12746 & 2450 \\
\hline 4.297 & 425 & 2620 \\
\hline N/A & N/A & N/A \\
\hline N/A & $\mathrm{N} / \mathrm{A}$ & $\mathrm{N} / \mathrm{A}$ \\
\hline 269.669 & 33403 & 3250 \\
\hline 0.83 & 34 & 3210 \\
\hline 5.79 & 495 & 2350 \\
\hline 27.691 & 3274 & 2460 \\
\hline 38.675 & 4665 & 3100 \\
\hline 4.256 & 414 & 2700 \\
\hline 2.784 & 244 & 2300 \\
\hline 61.307 & 7562 & 2860 \\
\hline 9.08 & 1022 & 2510 \\
\hline 2.748 & 141 & 2410 \\
\hline 1.124 & 55 & 2770 \\
\hline 26.418 & 3020.192308 & 2715.769231 \\
\hline
\end{tabular}




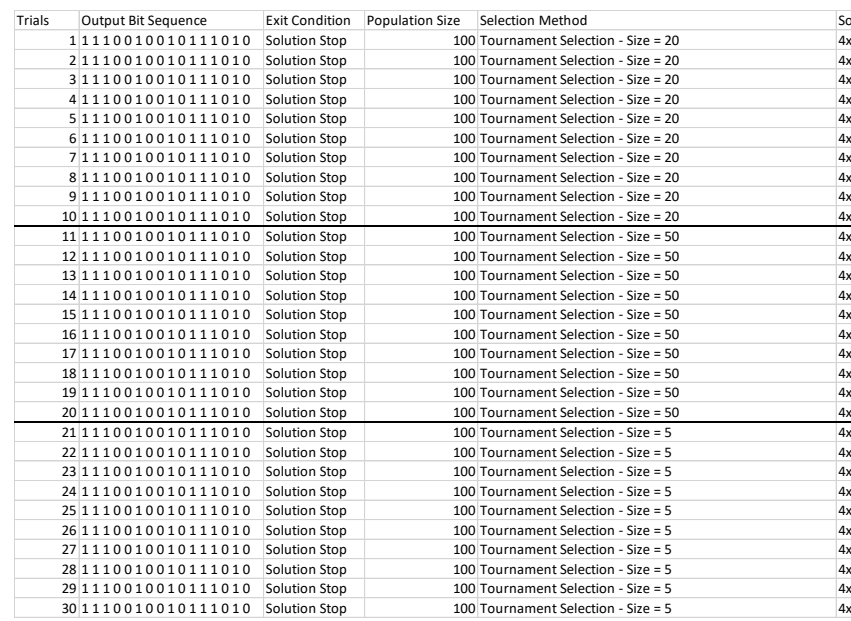

\begin{tabular}{|c|c|c|c|}
\hline Solution Space (array dimensions) & Crossover Technique & Crossover Rate Mutation Technique & Mutation Rate \\
\hline $4 \times 4$ & Single-point & 0.8 Random Gene Replacement & 0.2 \\
\hline $4 \times 4$ & Single-point & 0.8 Random Gene Replacement & \\
\hline $4 \times 4$ & Single-point & 0.8 Random Gene Replacement & \\
\hline $4 \times 4$ & Single-point & 0.8 Random Gene Replacement & \\
\hline $4 \times 4$ & Single-point & 0.8 Random Gene Replacement & 0.2 \\
\hline $4 \times 4$ & Single-point & 0.8 Random Gene Replacement & 0.2 \\
\hline $4 \times 4$ & Single-point & 0.8 Random Gene Replacement & \\
\hline $4 \times 4$ & Single-point & 0.8 Random Gene Replacement & \\
\hline $4 \times 4$ & Single-point & 0.8 Random Gene Replacement & \\
\hline $4 \times 4$ & Single-point & 0.8 Random Gene Replacement & 0.2 \\
\hline $4 \times 4$ & Single-point & 0.8 Random Gene Replacement & \\
\hline $4 \times 4$ & Single-point & 0.8 Random Gene Replacement & 0.2 \\
\hline $4 \times 4$ & Single-point & 0.8 Random Gene Replacement & 0.2 \\
\hline $4 \times 4$ & Single-point & 0.8 Random Gene Replacement & 0.2 \\
\hline $4 \times 4$ & Single-point & 0.8 Random Gene Replacement & \\
\hline $4 \times 4$ & Single-point & 0.8 Random Gene Replacement & 0.2 \\
\hline $4 \times 4$ & Single-point & 0.8 Random Gene Replacement & \\
\hline $4 \times 4$ & Single-point & 0.8 Random Gene Replacement & \\
\hline $4 \times 4$ & Single-point & 0.8 Random Gene Replacement & 0.2 \\
\hline $4 \times 4$ & Single-point & 0.8 Random Gene Replacement & \\
\hline $4 \times 4$ & Single-point & 0.8 Random Gene Replacement & \\
\hline $4 \times 4$ & Single-point & 0.8 Random Gene Replacement & 0.2 \\
\hline $4 \times 4$ & Single-point & 0.8 Random Gene Replacement & \\
\hline $4 \times 4$ & Single-point & 0.8 Random Gene Replacement & \\
\hline $4 \times 4$ & Single-point & 0.8 Random Gene Replacement & \\
\hline $4 \times 4$ & Single-point & 0.8 Random Gene Replacement & 0.2 \\
\hline $4 \times 4$ & Single-point & 0.8 Random Gene Replacement & \\
\hline $4 \times 4$ & Single-point & 0.8 Random Gene Replacement & 0.2 \\
\hline $4 \times 4$ & Single-point & 0.8 Random Gene Replacement & \\
\hline $4 \times 4$ & Single-point & 0.8 Random Gene Replacement & \\
\hline
\end{tabular}

\section{Computation Time (seconds)}

Generations

N/A

N/A N/A

\# Trials Prematurely Converged

\begin{tabular}{|c|c|c|c|c|c|}
\hline & 290.064 & 34827 & & 2810 & Average Computation Time \\
\hline N/A & & N/A & N/A & & 112.02125 \\
\hline & 126.953 & 15920 & & 2700 & Average \# Generations \\
\hline & 24.971 & 3056 & & 2860 & 13602.5 \\
\hline N/A & & N/A & N/A & & Average Solution Quality \\
\hline N/A & & N/A & N/A & & 2667.5 \\
\hline & 6.097 & 607 & & 2300 & \\
\hline N/A & & N/A & N/A & & \\
\hline $\mathrm{N} / \mathrm{A}$ & & $\mathrm{N} / \mathrm{A}$ & N/A & & \\
\hline & 18.25 & 2119 & & 2300 & \# Trials Prematurely Converged \\
\hline N/A & & N/A & N/A & & 3 \\
\hline & 1.223 & 45 & & 2460 & Average Computation Time \\
\hline & 1.647 & 103 & & 2450 & 22.94242857 \\
\hline N/A & & N/A & N/A & & Average \# Generations \\
\hline & 57.649 & 6995 & & 3450 & 2686.714286 \\
\hline N/A & & N/A & N/A & & Average Solution Quality \\
\hline & 71.876 & 8553 & & 3100 & 2988.571429 \\
\hline & 8.382 & 899 & & 2860 & \\
\hline & 1.57 & 93 & & 4300 & \\
\hline$\overline{N / A}$ & & $\mathrm{~N} / \mathrm{A}$ & $\mathrm{N} / \mathrm{A}$ & & \# Trials Prematurely Converged \\
\hline N/A & & N/A & N/A & & 4 \\
\hline N/A & & N/A & N/A & & Average Computation Time \\
\hline & 1.735 & 95 & & 2300 & 71.80283333 \\
\hline & 3.35 & 291 & & 2300 & Average \# Generations \\
\hline & 0.78 & 33 & & 2860 & 9008.5 \\
\hline & 51.603 & 6393 & & 2620 & Average Solution Quality \\
\hline & 28.237 & 3456 & & 3210 & 2623.333333 \\
\hline N/A & & N/A & N/A & & \\
\hline & 345.112 & 43783 & & 2450 & \\
\hline
\end{tabular}




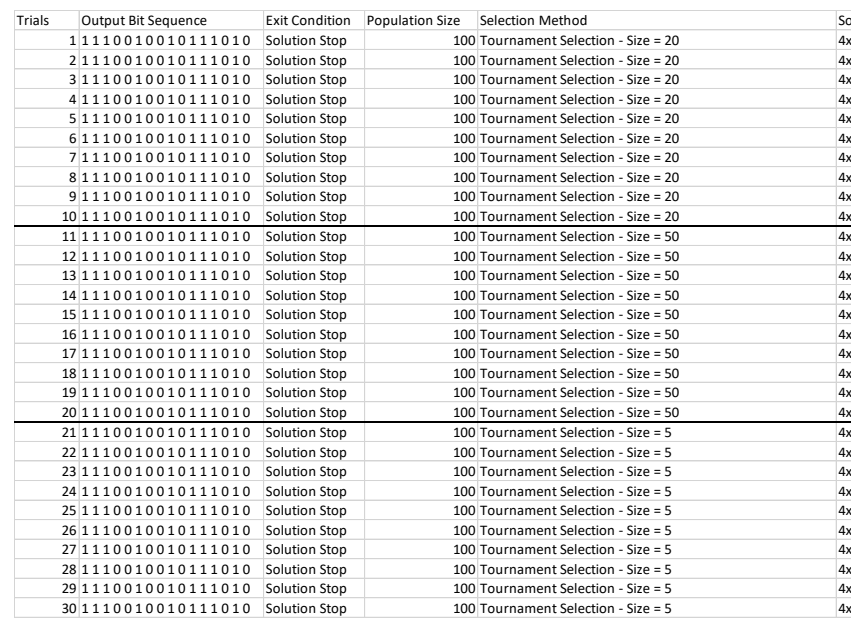

\begin{tabular}{|c|c|c|c|}
\hline Solution Space (array dimensions) & Crossover Technique & Crossover Rate Mutation Technique & Mutation Rate \\
\hline $4 \times 4$ & Uniform (rate $=0.5$ ) & 0.8 Random Gene Replacement & 0.2 \\
\hline $4 \times 4$ & Uniform (rate $=0.5$ ) & 0.8 Random Gene Replacement & 0.2 \\
\hline $4 \times 4$ & Uniform (rate $=0.5$ ) & 0.8 Random Gene Replacement & \\
\hline $4 \times 4$ & Uniform (rate $=0.5$ ) & 0.8 Random Gene Replacement & \\
\hline $4 \times 4$ & Uniform (rate $=0.5$ ) & 0.8 Random Gene Replacement & \\
\hline $4 \times 4$ & Uniform (rate $=0.5$ ) & 0.8 Random Gene Replacement & 0.2 \\
\hline $4 \times 4$ & Uniform (rate $=0.5$ ) & 0.8 Random Gene Replacement & \\
\hline $4 \times 4$ & Uniform (rate $=0.5$ ) & 0.8 Random Gene Replacement & 0.2 \\
\hline $4 \times 4$ & Uniform (rate $=0.5$ ) & 0.8 Random Gene Replacement & \\
\hline $4 \times 4$ & Uniform (rate $=0.5$ ) & 0.8 Random Gene Replacement & 0.2 \\
\hline $4 \times 4$ & Uniform (rate $=0.5$ ) & 0.8 Random Gene Replacement & \\
\hline $4 \times 4$ & Uniform (rate $=0.5$ ) & 0.8 Random Gene Replacement & \\
\hline $4 \times 4$ & Uniform (rate $=0.5$ ) & 0.8 Random Gene Replacement & \\
\hline $4 \times 4$ & Uniform (rate $=0.5$ ) & 0.8 Random Gene Replacement & 0.2 \\
\hline $4 \times 4$ & Uniform (rate $=0.5$ ) & 0.8 Random Gene Replacement & 0.2 \\
\hline $4 \times 4$ & Uniform (rate $=0.5$ ) & 0.8 Random Gene Replacement & \\
\hline $4 \times 4$ & Uniform (rate $=0.5$ ) & 0.8 Random Gene Replacement & \\
\hline $4 \times 4$ & Uniform (rate $=0.5$ ) & 0.8 Random Gene Replacement & \\
\hline $4 \times 4$ & Uniform (rate $=0.5$ ) & 0.8 Random Gene Replacement & \\
\hline $4 \times 4$ & Uniform (rate $=0.5$ ) & 0.8 Random Gene Replacement & 0.2 \\
\hline $4 \times 4$ & Uniform (rate $=0.5$ ) & 0.8 Random Gene Replacement & \\
\hline $4 \times 4$ & Uniform (rate $=0.5$ ) & 0.8 Random Gene Replacement & \\
\hline $4 \times 4$ & Uniform (rate $=0.5$ ) & 0.8 Random Gene Replacement & \\
\hline $4 \times 4$ & Uniform (rate $=0.5$ ) & 0.8 Random Gene Replacement & \\
\hline $4 \times 4$ & Uniform (rate $=0.5$ ) & 0.8 Random Gene Replacement & 0.2 \\
\hline $4 \times 4$ & Uniform (rate $=0.5$ ) & 0.8 Random Gene Replacement & \\
\hline $4 \times 4$ & Uniform (rate $=0.5$ ) & 0.8 Random Gene Replacement & \\
\hline $4 \times 4$ & Uniform (rate $=0.5$ ) & 0.8 Random Gene Replacement & 0.2 \\
\hline $4 \times 4$ & Uniform (rate = 0.5) & 0.8 Random Gene Replacement & \\
\hline $4 \times 4$ & Uniform (rate $=0.5$ ) & 0.8 Random Gene Replacement & \\
\hline
\end{tabular}

Computation Time (seconds) Generations Solution Quality (fitness) \# Trials Prematurely Converged

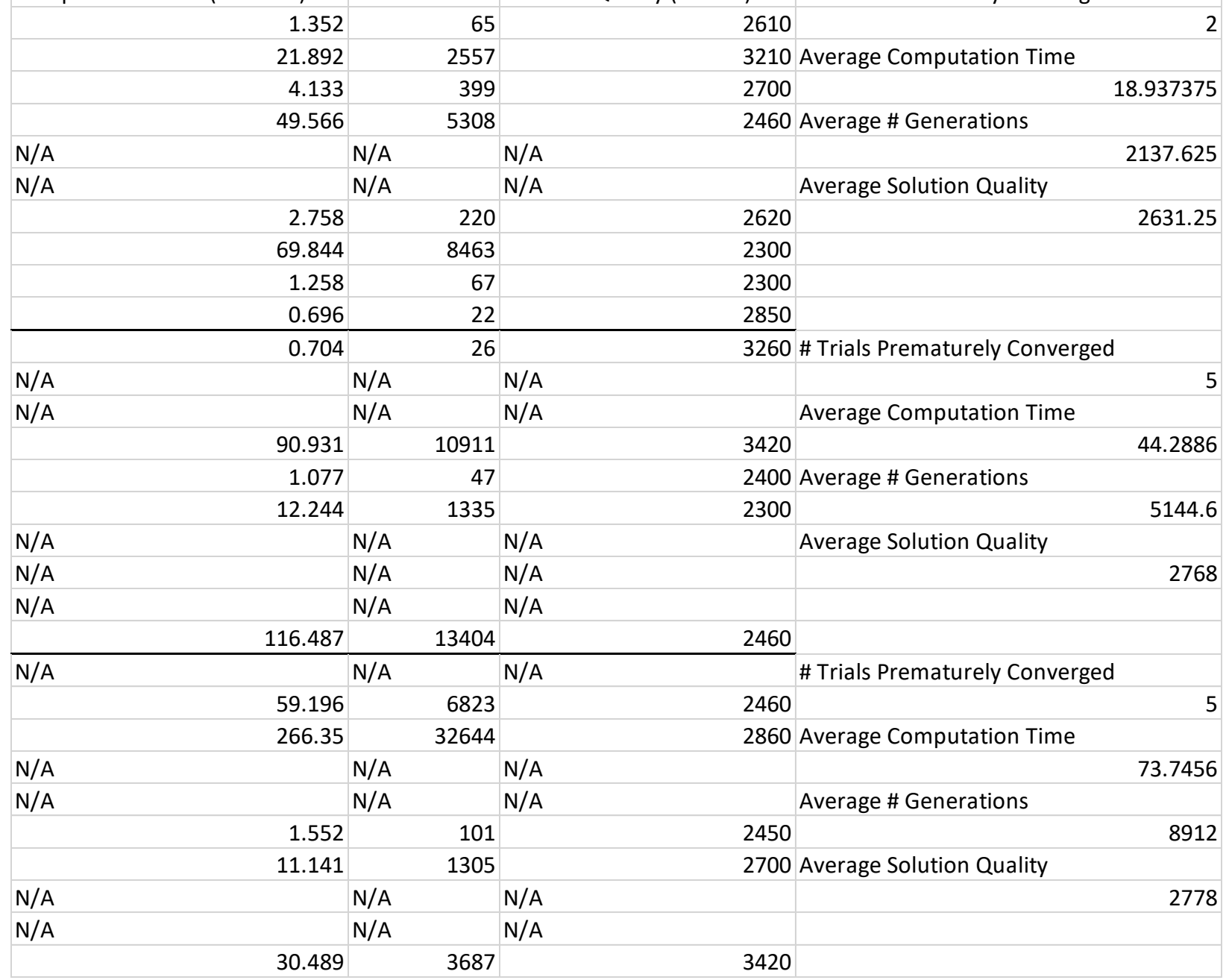



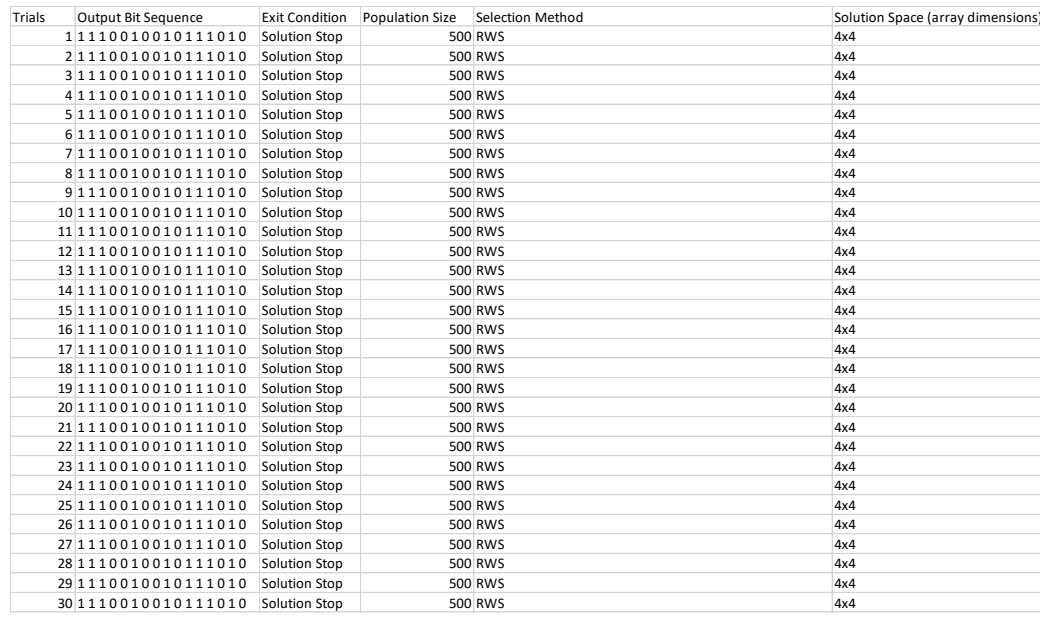

Crossover Technique Crossover Rate Mutation Technique

ifform (rate $=0.5$ ) $\quad 0.8$ Random Gene Replacement Uniform (rate $=0.5$ ) $\quad 0.8$ Random Gene Replacement Uniform $($ rate $=0.5) \quad 0.8$ Random Gene Replacement Uniform (rate $=0.5$ ) 0.8 Random Gene Replacement Uniform (rate $=0.5$ ) $\quad 0.8$ Random Gene Replacement Uniform (rate $=0.5$ ) $\quad 0.8$ Random Gene Replacement Uniform (rate $=0.5$ ) $\quad 0.8$ Random Gene Replacement Uniform (rate $=0.5$ ) $\quad 0.8$ Random Gene Replacement Uniform (rate $=0.5$ ) 0.8 Random Gene Replacement Uniform (rate $=0.5) \quad 0.8$ Random Gene Replacement Uniform $($ rate $=0.5) \quad 0.8$ Random Gene Replament Uniform (rate $=0.5) \quad 0.8$ Random Ge Replacement Uniform (rate $=0.5$ ) 0.8 Random Gene Replacement Uniform (rate $=0.5$ ) $\quad 0.8$ Random Gene Replacement Uniform (rate $=0.5$ ) $\quad 0.8$ Random Gene Replacement Uniform (rate $=0.5) \quad 0.8$ Random Gene Replacement Uniform (rate $=0.5$ ) $\quad 0.8$ Random Gene Replacement Uniform (rate $=0.5$ ) $\quad 0.8$ Random Gene Replacement Uniform (rate $=0.5) \quad 0.8$ Random Gene Replacement Uniform (rate $=0.5) \quad 0.8$ Random Gene Replacement Uniform (rate $=0.5) \quad 08$ Random Gene Replocement Uniform (rate $=0.5$ ) 0.8 Random Gene Replacement Uniform (rate $=0.5$ ) $\quad 0.8$ Random Gene Replacement Uniform (rate $=0.5) \quad 0.8$ Random Gene Replacement 0.8 Random Gene Replacement

$\begin{array}{ll}\text { Uniform }(\text { rate }=0.5) & 0.8 \text { Random Gene Replacement } \\ \text { Uniform (rate }=0.5) & 0.8 \text { Random Gene Replacement }\end{array}$ Uniform (rate $=0.5$

Mutation Rate 500 RWS Computation Time (seconds) Generations Solution Quality (fitness)

\begin{tabular}{|c|c|c|}
\hline 70.335 & 1832 & 2300 \\
\hline 23.569 & 626 & 2460 \\
\hline 40.959 & 1096 & 2510 \\
\hline 47.114 & 1248 & 2750 \\
\hline 43.166 & 1139 & 4010 \\
\hline 56.656 & 1520 & 2970 \\
\hline 23.677 & 622 & 3100 \\
\hline 49.149 & 1323 & 3170 \\
\hline 57.348 & 1534 & 3420 \\
\hline 82.981 & 2259 & 2700 \\
\hline 8.208 & 200 & 2910 \\
\hline 84.693 & 2310 & 2450 \\
\hline 17.844 & 451 & 3180 \\
\hline 18.347 & 479 & 3370 \\
\hline 16.036 & 411 & 2700 \\
\hline 87.229 & 2387 & 3100 \\
\hline 54.41 & 1475 & 3100 \\
\hline 44.422 & 1195 & 3260 \\
\hline 9.721 & 239 & 3420 \\
\hline 32.781 & 883 & 2850 \\
\hline 38.184 & 1031 & 2610 \\
\hline 25.071 & 655 & 2780 \\
\hline 55.305 & 1483 & 2450 \\
\hline 20.402 & 530 & 2450 \\
\hline 32.981 & 875 & 2450 \\
\hline 35.64 & 944 & 3420 \\
\hline 3.076 & 59 & 3900 \\
\hline 3.878 & 79 & 3310 \\
\hline 110.122 & 3012 & 3100 \\
\hline 20.948 & 546 & 2610 \\
\hline 40.47506667 & 1081.433333 & 2960.333333 \\
\hline
\end{tabular}



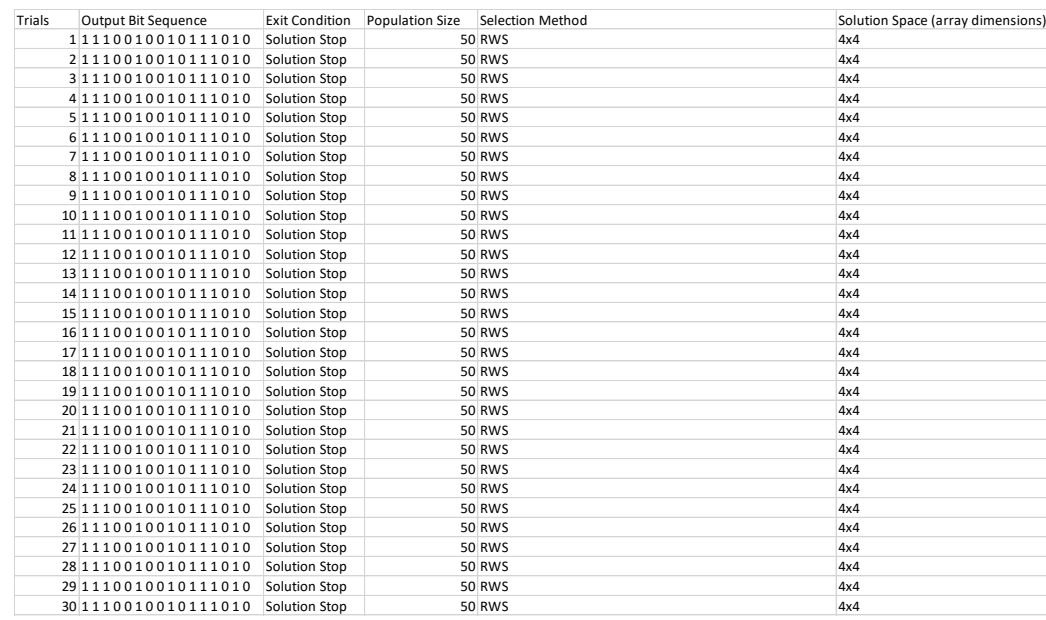

Crossover Technique Crossover Rate Mutation Technique

0.8 Random Gene Replacement Uniform (rate $=0.5) \quad 0.8$ Random Gene Replacement Uniform (rate $=0.5) \quad 0.8$ Random Gene Replacement Uniform (rate $=0.5$ ) 0.8 Random Gene Replacement Uniform (rate $=0.5$ ) $\quad 0.8$ Random Gene Replacement Uniform (rate $=0.5$ ) $\quad 0.8$ Random Gene Replacement Uniform (rate $=0.5$ ) $\quad 0.8$ Random Gene Replacement Uniform (rate $=0.5$ ) $\quad 0.8$ Random Gene Replacement Uniform (rate $=0.5) \quad 0.8$ Random Gene Replacement Uniform (rate $=0.5) \quad 0.8$ Random Gene Replacement Uniform (rate $=0.5) \quad 0.8$ Random Gene Replacent Uniform (rate $=0.5) \quad 0.8$ Random Gene Replacement Uniform (rate $=0.5$ ) $\quad 0.8$ Random Gene Replacement Uniform (rate $=0.5$ ) $\quad 0.8$ Random Gene Replacement Uniform (rate $=0.5$ ) $\quad 0.8$ Random Gene Replacement Uniform (rate $=0.5$ ) $\quad 0.8$ Random Gene Replacement Uniform (rate $=0.5$ ) $\quad 0.8$ Random Gene Replacement Uniform (rate $=0.5) \quad 0.8$ Random Gene Replacement Uniform (rate $=0.5) \quad 0.8$ Random Gene Replacement Uniform (rate $=0.5) \quad 0.8$ Random Gene Replacement Uniform (rate $=0.5) \quad 08$ Random Gene Replacement Uniform (rate $=0.5$ ) 0.8 Random Gene Replacement Uniform (rate $=0.5$ ) 0.8 Random Gene Replacement Uniform (rate $=0.5) \quad 0.8$ Random Gene Replacement 0.8 Random Gene Replacement $\begin{array}{ll}\text { Uniform }(\text { rate }=0.5) & 0.8 \text { Random Gene Replacement } \\ \text { Uniform (rate }=0.5) & 0.8 \text { Random Gene Replacement }\end{array}$

\begin{tabular}{|r|r|r|}
\hline Computation Time (seconds) & Generations & Solution Quality (fitness) \\
\hline 3.974 & 246 & 2410 \\
\hline 6.589 & 599 & 2780 \\
\hline 1.402 & 61 & 2450 \\
\hline 18.203 & 2053 & 2300 \\
\hline 44.409 & 5496 & 3260 \\
\hline 7.833 & 692 & 2450 \\
\hline 6.115 & 600 & 2700 \\
\hline 15.164 & 1781 & 2860 \\
\hline 23.948 & 2844 & 2860 \\
\hline 2.738 & 170 & 3050 \\
\hline 7.068 & 701 & 2780 \\
\hline 25.888 & 3177 & 3180 \\
\hline 1.831 & 96 & 3150 \\
\hline 11.721 & 1355 & 3260 \\
\hline 2.592 & 174 & 2610 \\
\hline 4.511 & 391 & 3580 \\
\hline 7.693 & 770 & 2460 \\
\hline 4.088 & 351 & 2250 \\
\hline 2.243 & 140 & 2860 \\
\hline 6.167 & 600 & 2250 \\
\hline 6.917 & 717 & 2620 \\
\hline 6.837 & 665 & 2410 \\
\hline 6.476 & 489 & 2770 \\
\hline 10.594 & 1144 & 2610 \\
\hline 22.076 & 3459 & 2410 \\
\hline 1.809 & 180 & 2610 \\
\hline 2.294 & 331 & 2300 \\
\hline 3.64 & 616 & 2610 \\
\hline 0.787 & 56 & 2810 \\
\hline 1.268 & 125 & 3260 \\
\hline 8.85833333 & 1002.633333 & 2730.333333 \\
\hline & & \\
\hline & &
\end{tabular}



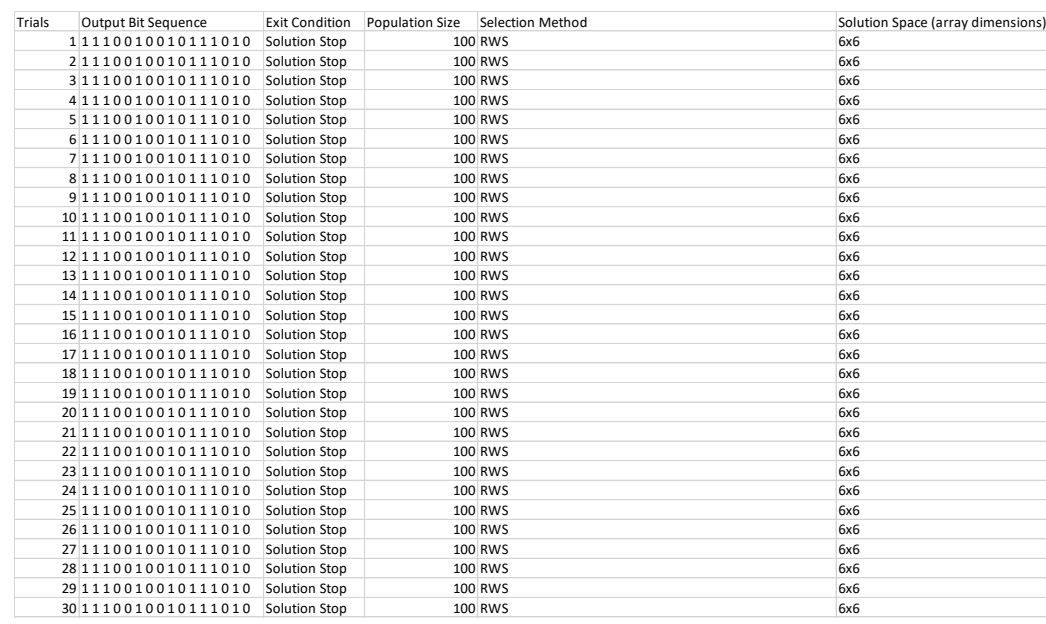

Crossover Technique Crossover Rate Mutation Technique

0.8 Random Gene Replacement 0.8 Random Gene Replacement Uniform (rate $=0.5) \quad 0.8$ Random Gene Replacement Uniform (rate $=0.5$ ) 0.8 Random Gene Replacement Uniform (rate $=0.5$ ) $\quad 0.8$ Random Gene Replacement Uniform (rate $=0.5$ ) $\quad 0.8$ Random Gene Replacement Uniform (rate $=0.5$ ) $\quad 0.8$ Random Gene Replacement Uniform (rate $=0.5$ ) $\quad 0.8$ Random Gene Replacement Uniform (rate $=0.5$ ) $\quad 0.8$ Random Gene Replacement Uniform (rate $=0.5) \quad 0.8$ Random Gene Replacement Uniform $($ rate $=0.5) \quad 0.8$ Random Gene Replament Uniform (rate $=0.5) \quad 0.8$ Random Gene Replacement Uniform (rate $=0.5$ ) 0.8 Random Gene Replacement Uniform (rate $=0.5$ ) $\quad 0.8$ Random Gene Replacement Uniform (rate $=0.5$ ) $\quad 0.8$ Random Gene Replacement Uniform (rate $=0.5$ ) $\quad 0.8$ Random Gene Replacement Uniform (rate $=0.5$ ) $\quad 0.8$ Random Gene Replacement Uniform (rate $=0.5$ ) $\quad 0.8$ Random Gene Replacement Uniform (rate $=0.5) \quad 0.8$ Random Gene Replacement Uniform (rate $=0.5) \quad 0.8$ Random Gene Replacement Uniform (rate $=05) \quad 08$ Random Gene Replacement Uniform (rate $=0.5$ ) $\quad 0.8$ Random Gene Replacement Uniform (rate $=0.5$ ) $\quad 0.8$ Random Gene Replacement Uniform (rate $=0.5$ ) $\quad 0.8$ Random Gene Replacement 0.8 Random Gene Replacement Uniform (rate $=0.5$ ) $\quad 0.8$ Random Gene Replacement 0.8 Random Gene Replaceme

\begin{tabular}{|c|c|c|}
\hline Computation Time (seconds) & Generations & Solution Quality (fitness) \\
\hline 3.62 & 120 & 5730 \\
\hline 6.873 & 302 & 6320 \\
\hline 16.629 & 790 & 7500 \\
\hline 10.046 & 456 & 4750 \\
\hline 2.383 & 84 & 3050 \\
\hline 2.796 & 99 & 4350 \\
\hline 1.645 & 51 & 3670 \\
\hline 14.83 & 717 & 6060 \\
\hline 18.858 & 922 & 5100 \\
\hline 23.464 & 1210 & 4100 \\
\hline 7.774 & 370 & 3450 \\
\hline 35.572 & 1888 & 3810 \\
\hline 13.624 & 245 & 4820 \\
\hline 4.069 & 177 & 3810 \\
\hline 5.67 & 254 & 3610 \\
\hline 4.547 & 189 & 5780 \\
\hline 2.459 & 86 & 6600 \\
\hline 6.484 & 292 & 5090 \\
\hline 7.633 & 356 & 3250 \\
\hline 29.928 & 1581 & 4260 \\
\hline 8.721 & 169 & 3450 \\
\hline 3.563 & 139 & 3360 \\
\hline 17.076 & 866 & 5210 \\
\hline 5.239 & 214 & 3450 \\
\hline 15.413 & 766 & 3820 \\
\hline 2.05 & 69 & 5570 \\
\hline 12.899 & 600 & 4310 \\
\hline 1.021 & 20 & 5030 \\
\hline 15.656 & 750 & 5520 \\
\hline 5.134 & 225 & 5960 \\
\hline 10.1892 & 466.9 & 4693 \\
\hline
\end{tabular}



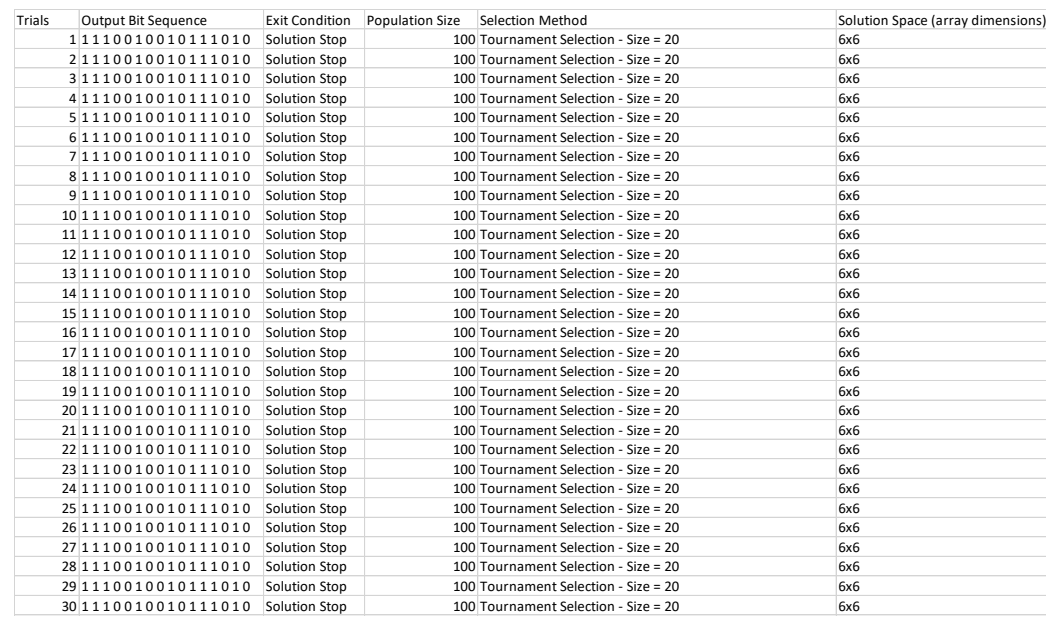

Crossover Technique Crossover Rate Mutation Technique

0.8 Random Gene Replacement $\begin{array}{ll}\text { Single-point } & 0.8 \text { Random Gene Replacement } \\ \text { Single-point } & 0.8 \text { Random Gene Replacement }\end{array}$ $\begin{array}{ll}0.8 \text { Random Gene Replacement } \\ \text { Single-point } & 0.8 \text { Random Gene Replacement }\end{array}$ Single-point $\quad 0.8$ Random Gene Replacement Single-point 0.8 Random Gene Replacement Single-point 0.8 Random Gene Replacement Single-point 0.8 Random Gene Replacement $\begin{array}{ll}\text { Single-point } & 0.8 \text { Random Gene Replacement } \\ \text { Single-point } & 0.8 \text { Random Gene Replacement }\end{array}$ $\begin{array}{ll}\text { Single-point } & 0.8 \text { Random Gene Replacement } \\ \text { Single-point } & 0.8 \text { Random Gene Replacement }\end{array}$ \begin{tabular}{ll} 
Single-point & 0.8 Random Gene Replacement \\
\hline Single-point & 0.8 Random Gene Replacement
\end{tabular} $\begin{array}{ll}\text { single-point } & 0.8 \text { Random Gene Replacement } \\ \text { Single-point } & 0.8 \text { Random Gene Replacement }\end{array}$ Single-point $\quad 0.8$ Random Gene Replacement Single-point 0.8 Random Gene Replacement Single-point 0.8 Random Gene Replacement Single-point 0.8 Random Gene Replacement $\begin{array}{ll}\text { Single-point } & 0.8 \text { Random Gene Replacement } \\ \text { Single-point } & 0.8 \text { Random Gene Replacement }\end{array}$ $\begin{array}{ll}\text { Single-point } & 0.8 \text { Random Gene Replacement } \\ \text { Single-point } & 0.8 \text { Random Gene Replacement }\end{array}$ $\begin{array}{ll}\text { Single-point } & 0.8 \text { Random Gene Replacement } \\ \text { Single-point } & 0.8 \text { Random Gene Replacement }\end{array}$ $\begin{array}{ll}\text { Single-point } & 0.8 \text { Random Gene Replacement } \\ \text { Single-point } & 0.8 \text { Random Gene Replacement }\end{array}$ $\begin{array}{ll}\text { Single-point } & 0.8 \text { Random Gene Replacement } \\ \text { Single-point } & 0.8 \text { Random Gene Replacement }\end{array}$ Single-point $\quad 0.8$ Random Gene Replacement $\begin{array}{ll}0.8 \text { Random Gene Replacement } \\ \text { Single-point } & 0.8 \text { Random Gene Replacement }\end{array}$ single-point 0.8 Random Gene Replacement Single-point 0.8 Random Gene Replacement $\begin{array}{ll}\text { Single-point } & 0.8 \text { Random Gene Replacement } \\ \text { Single-point } & 0.8 \text { Random Gene Replacement }\end{array}$ \begin{tabular}{ll} 
Single-point & 0.8 Random Gene Replacement \\
\hline Single-point & 0.8 Random Gene Replacement
\end{tabular}

\begin{tabular}{|c|c|c|}
\hline Computation Time (seconds) & Generations & Solution Quality (fitness) \\
\hline 1.806 & 51 & 3210 \\
\hline 275.101 & 14821 & 4260 \\
\hline 3.483 & 88 & 3200 \\
\hline 2.126 & 59 & 6110 \\
\hline 8.26 & 382 & 3460 \\
\hline 2.622 & 92 & 3770 \\
\hline 14.424 & 718 & 4000 \\
\hline 42.484 & 2092 & 4700 \\
\hline 2.222 & 53 & 3300 \\
\hline 8.915 & 402 & 3620 \\
\hline 55.666 & 2940 & 8120 \\
\hline 1.482 & 36 & 8250 \\
\hline 4.324 & 156 & 7120 \\
\hline 9.411 & 445 & 4010 \\
\hline 6.241 & 238 & 10280 \\
\hline 8.089 & 362 & 5120 \\
\hline 242.825 & 12304 & 4600 \\
\hline 1.053 & 17 & 3110 \\
\hline 9.422 & 437 & 7220 \\
\hline 3.139 & 112 & 8350 \\
\hline 127.766 & 6409 & 5320 \\
\hline 11.24 & 549 & 7660 \\
\hline 10.633 & 482 & 3770 \\
\hline 1.892 & 55 & 6760 \\
\hline 111.639 & 5491 & 4100 \\
\hline 1.767 & 51 & 8970 \\
\hline 2.436 & 79 & 7610 \\
\hline 1.188 & 21 & 4960 \\
\hline 7.513 & 317 & 4160 \\
\hline 2.62 & 91 & 4990 \\
\hline 32.7263 & 1645 & 5470.333333 \\
\hline
\end{tabular}



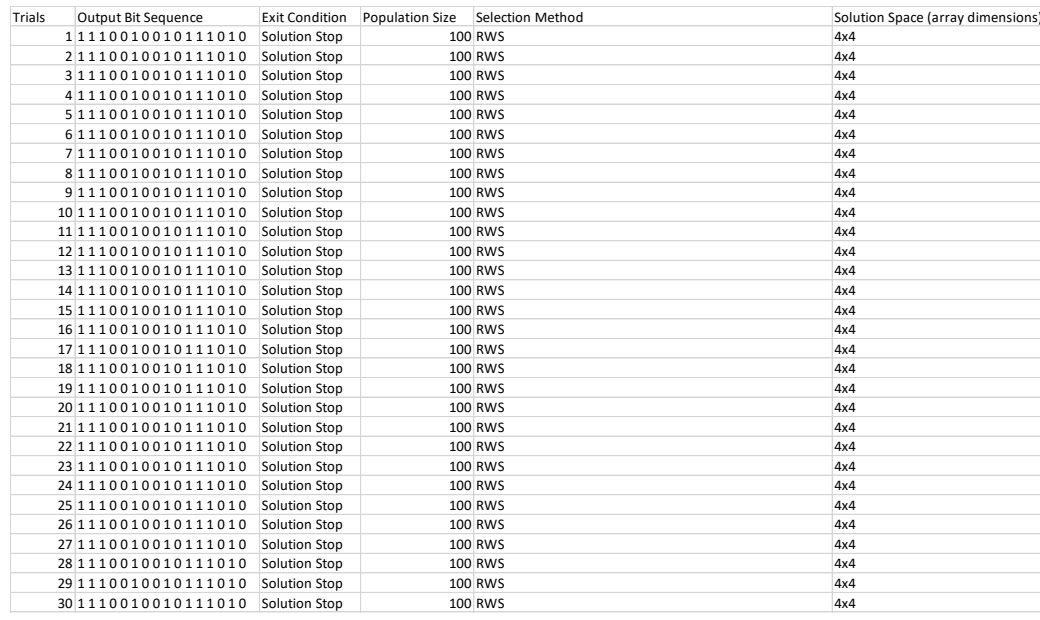

Crossover Technique Crossover Rate Mutation Technique

0.5 Random Gene Replacement 0.5 Random Gene Replacement Uniform $(x$ te $=0.5) \quad 0.5$ Rand Uniform (rate $=0.5) \quad 0.5$ Random Gene Replacement Uniform (rate $=0.5$ ) $\quad 0.5$ Random Gene Replacement Uniform (rate $=0.5$ ) $\quad 0.5$ Random Gene Replacement Uniform (rate $=0.5$ ) $\quad 0.5$ Random Gene Replacement Uniform (rate $=0.5$ ) $\quad 0.5$ Random Gene Replacement Uniform (rate $=0.5$ ) $\quad 0.5$ Random Gene Replacement Uniform (rate $=0.5) \quad 0.5$ Random Gene Replacement Uniform (rate $=0.5) \quad 0.5$ Random Gene Repcent Uniform (rate $=0.5) \quad 0.5$ Random Gene Replacement Uniform (rate $=0.5$ ) 0.5 Random Gene Replacement Uniform (rate $=0.5$ ) $\quad 0.5$ Random Gene Replacement Uniform (rate $=0.5$ ) $\quad 0.5$ Random Gene Replacement Uniform (rate $=0.5$ ) $\quad 0.5$ Random Gene Replacement Uniform (rate $=0.5$ ) $\quad 0.5$ Random Gene Replacement Uniform (rate $=0.5$ ) $\quad 0.5$ Random Gene Replacement Uniform (rate $=0.5) \quad 0.5$ Random Gene Replacement Uniform (rate $=0.5) \quad 0.5$ Random Gene Replacement 0.5 Random Gene Replacement Uniform (rate $=0.5$ ) 0.5 Random Gene Replacement Uniform (rate $=0.5$ ) $\quad 0.5$ Random Gene Replacement Uniform (rate $=0.5$ ) $\quad 0.5$ Random Gene Replacement 0.5 Random Gene Replacement Uniform (rate $=0.5$ ) $\quad 0.5$ Random Gene Replacement 0.5 Random Gene Replacemen

Mutation Rate Uniform (rate $=0.5$ )

\begin{tabular}{|c|c|c|}
\hline Computation Time (seconds) & Generations & Solution Quality (fitness) \\
\hline 40.563 & 4257 & 3420 \\
\hline 74.638 & 7928 & 2450 \\
\hline 82.418 & 8438 & 2300 \\
\hline 69.649 & 7472 & 2510 \\
\hline 135.463 & 14658 & 3420 \\
\hline 24.988 & 2625 & 2770 \\
\hline 99.795 & 10818 & 2700 \\
\hline 45.932 & 4850 & 2300 \\
\hline 3.877 & 315 & 2620 \\
\hline 154.192 & 16858 & 2300 \\
\hline 6.441 & 609 & 2620 \\
\hline 24.979 & 2571 & 2300 \\
\hline 6.149 & 566 & 2750 \\
\hline 39.749 & 4193 & 3150 \\
\hline 16.676 & 1270 & 2860 \\
\hline 43.491 & 4701 & 3420 \\
\hline 77.614 & 8375 & 3100 \\
\hline 42.601 & 4554 & 2610 \\
\hline 37.777 & 3867 & 2800 \\
\hline 14.887 & 1540 & 2450 \\
\hline 110.037 & 11905 & 3660 \\
\hline 61.711 & 6467 & 2800 \\
\hline 5.735 & 525 & 3100 \\
\hline 79.067 & 8589 & 2620 \\
\hline 34.516 & 3585 & 3310 \\
\hline 5.313 & 470 & 2450 \\
\hline 3.854 & 305 & 2700 \\
\hline 53.439 & 5586 & 2610 \\
\hline 24.724 & 2485 & 2770 \\
\hline 26.889 & 2787 & 2970 \\
\hline 48.2388 & 5105.633333 & 2794.666667 \\
\hline
\end{tabular}



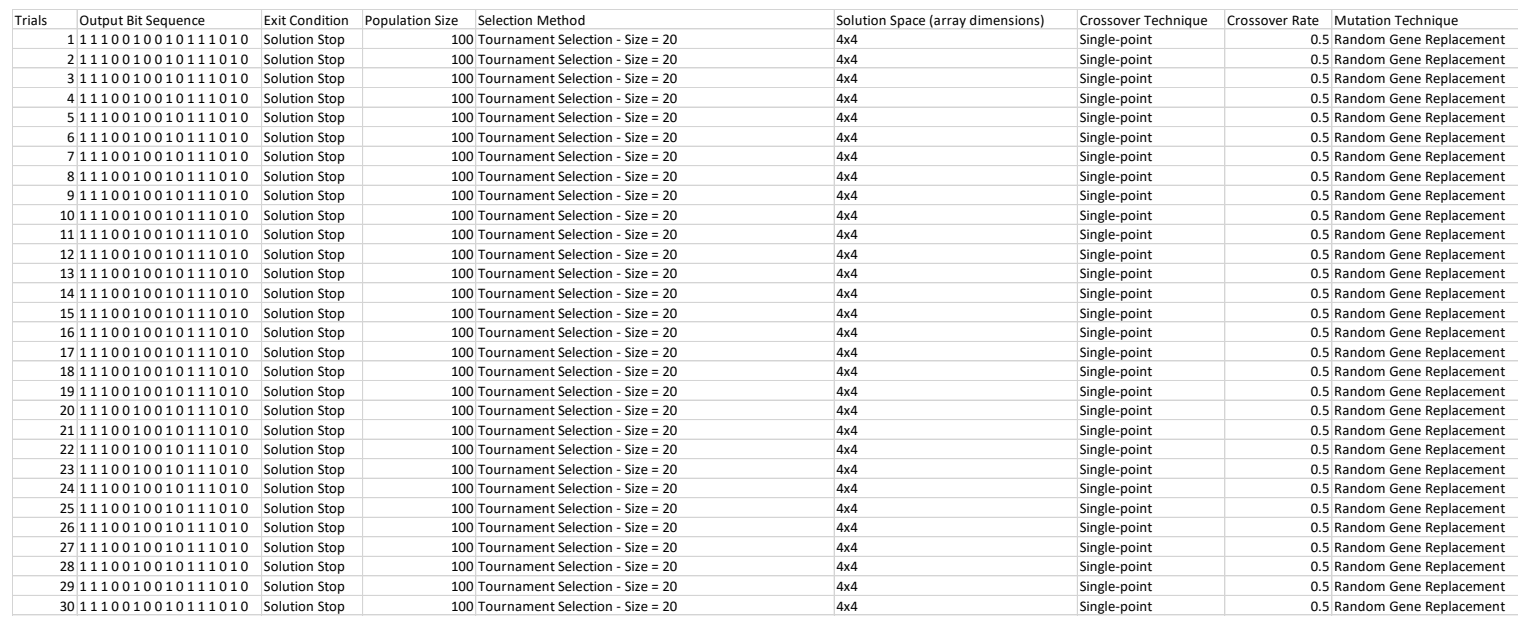

Mutation Rate

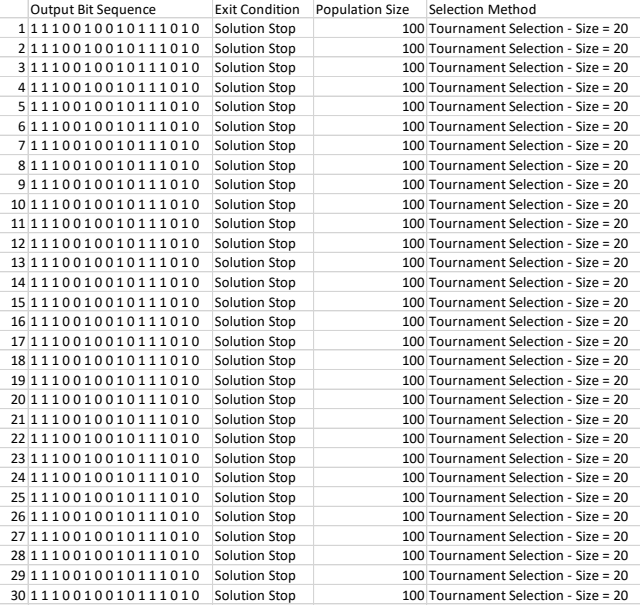

\section{Computation Time (seconds)}

N/A

Generations Solution Quality (fitness)

$N / A$

N/A

N/A

N/A

$N / A$

$N / A$

1.332

$\mathrm{N} / \mathrm{A}$

N/A

$\begin{array}{lrr}1.332 & 44 & 2860\end{array}$

2.006

109

2730

4.961

427

3900

10.882

N/A

1111

2300

年

N/A

12.413

0.577

2.228

9.79

N/A

N/A

2300

2460

4220

2460

$\mathrm{N} / \mathrm{A}$

1008

N/A

$N / A$

N/A

N/A

48.05

25.81

72.089

19.762

4.771

2.243

N/A

N/A

5327

N/A

N/A

2.519

2726

3020

2300

4066

2460

1882

3100

376

2460

149

3260

N/A

N/A

N/A

131

2300

65.606

149.816

5.101

0.501

73.743

N/A

4.531 N/A 406

N/A

$7324 \quad 2300$

\begin{tabular}{|r|r|}
17324 & 3310 \\
\hline 437
\end{tabular}

$437 \quad 2300$

\begin{tabular}{r|r}
9 & 3020 \\
\hline 8229 & 3100
\end{tabular}

$\begin{array}{ll}24.7017619 & 2489.666667\end{array}$

3420 


\subsection{Post-Solution Trial Results}

\subsubsection{Post-Solution Summary}

\begin{tabular}{|c|c|c|c|c|c|c|c|}
\hline Trial Set & Exit Condition & Population Selection Method & Solution Space (array dimensions) & Crossover Technique & Crossover Rate & Mutation Technique & Mutation Rate \\
\hline A & 300 seconds & 100 RWS & $4 \times 4$ & Single-point & 0.8 & 8 Random gene replacement & 0.2 \\
\hline B & 300 seconds & 100 RWS & $4 \times 4$ & Uniform (rate $=0.5$ ) & 0.8 & 8 Random gene replacement & 0.2 \\
\hline C & 300 seconds & $100 \mathrm{TS}(1)$ & $4 \times 4$ & Single-point & 0.8 & 8 Random gene replacement & 0.2 \\
\hline D & 300 seconds & 100 RWS & $4 \times 4$ & Single-point & 0.8 & 8 Random gene replacement & 0.2 \\
\hline $\mathrm{E}$ & 300 seconds & $100 \mathrm{TS}(20)$ & $4 \times 4$ & Single-point & 0.8 & 8 Random gene replacement & 0.2 \\
\hline $\mathrm{F}$ & 300 seconds & $100 \mathrm{TS}(50)$ & $4 \times 4$ & Single-point & 0.8 & 8 Random gene replacement & 0.2 \\
\hline G & 300 seconds & $100 \mathrm{TS}(5)$ & $4 \times 4$ & Single-point & 0.8 & 8 Random gene replacement & 0.2 \\
\hline $\mathrm{H}$ & 300 seconds & $100 \mathrm{TS}(20)$ & $4 \times 4$ & Uniform (rate $=0.5$ ) & 0.8 & 8 Random gene replacement & 0.2 \\
\hline I & 300 seconds & $100 \mathrm{TS}(50)$ & $4 \times 4$ & Uniform (rate $=0.5$ ) & 0.8 & 8 Random gene replacement & 0.2 \\
\hline J & 300 seconds & $100 \mathrm{TS}(5)$ & $4 \times 4$ & Uniform (rate $=0.5$ ) & 0.8 & 8 Random gene replacement & 0.2 \\
\hline $\mathrm{K}$ & 300 seconds & 500 RWS & $4 \times 4$ & Uniform (rate $=0.5$ ) & 0.8 & 8 Random gene replacement & 0.2 \\
\hline $\mathrm{L}$ & 300 seconds & 50 RWS & $4 \times 4$ & Uniform (rate $=0.5$ ) & 0.8 & 8 Random gene replacement & 0.2 \\
\hline M & 1382 seconds & 500 RWS & $4 \times 4$ & Uniform (rate $=0.5$ ) & 0.8 & 8 Random gene replacement & 0.2 \\
\hline $\mathrm{N}$ & 290 seconds & 50 RWS & $4 \times 4$ & Uniform (rate $=0.5$ ) & 0.8 & 8 Random gene replacement & 0.2 \\
\hline 0 & 300 seconds & 500 RWS & $4 \times 4$ & Single-point & 0.8 & 8 Random gene replacement & 0.2 \\
\hline $\mathrm{P}$ & 300 seconds & $500 \mathrm{TS}(1)$ & $4 \times 4$ & Single-point & 0.8 & 8 Random gene replacement & 0.2 \\
\hline Q & 1000 seconds & 100 RWS & $4 \times 4$ & Single-point & 0.8 & 8 Random gene replacement & 0.2 \\
\hline $\mathrm{R}$ & 100 seconds & 100 RWS & $4 \times 4$ & Single-point & 0.8 & 8 Random gene replacement & 0.2 \\
\hline $\mathrm{s}$ & 50 seconds & 100 RWS & $4 \times 4$ & Single-point & 0.8 & 8 Random gene replacement & 0.2 \\
\hline $\mathrm{T}$ & 1000 seconds & $100 \mathrm{TS}(1)$ & $4 \times 4$ & Single-point & 0.8 & 8 Random gene replacement & 0.2 \\
\hline $\mathrm{U}$ & 100 seconds & $100 \mathrm{TS}(1)$ & $4 \times 4$ & Single-point & 0.8 & 8 Random gene replacement & 0.2 \\
\hline $\mathrm{V}$ & 50 seconds & $100 \mathrm{TS}(1)$ & $4 \times 4$ & Single-point & 0.8 & 8 Random gene replacement & 0.2 \\
\hline W & 300 seconds & 100 RWS & $4 \times 4$ & Single-point & 0.5 & 5 Random gene replacement & 0.5 \\
\hline $\mathrm{x}$ & 1258.5 seconds & $500 \mathrm{TS}(1)$ & $4 \times 4$ & Single-point & 0.8 & 8 Random gene replacement & 0.2 \\
\hline $\mathrm{Y}$ & 156.12 seconds & $50 \mathrm{TS}(1)$ & $4 \times 4$ & Single-point & 0.8 & 8 Random gene replacement & 0.2 \\
\hline Z & 300 seconds & $100 \mathrm{TS}(1)$ & $4 \times 4$ & Uniform (rate $=0.5$ ) & 0.8 & 8 Random gene replacement & 0.2 \\
\hline AA & 300 seconds & $100 \mathrm{TS}(1)$ & $4 \times 4$ & Uniform (rate $=0.5$ ) & 0.5 & 5 Random gene replacement & 0.5 \\
\hline BB & 1381.2 seconds & 500 RWS & $4 \times 4$ & Single-point & 0.8 & 8 Random gene replacement & 0.2 \\
\hline
\end{tabular}

Trial Set Average Starting Fitness Average Ending Fitness Average Difference Average Generations Premature Convergence (30 trials)

\begin{tabular}{|c|c|c|c|c|c|}
\hline & & & & & \\
\hline A & 2998.6 & 4687 & 1688.4 & 26150 & 2 \\
\hline B & 2856.6 & 4098.6 & 1242 & 29055 & c \\
\hline C & 2766.6 & 4785.6 & 2019 & 29740 & 7 \\
\hline D & 2837.3 & 5212.6 & 2375.3 & 31631.7 & 5 \\
\hline E & 2739 & 3425 & 686 & & 3 (10 trials) \\
\hline $\mathrm{F}$ & 2731 & 3416 & 685 & & 6 (10 trials) \\
\hline G & 2956 & 3358 & 402 & & 4 (10 trials) \\
\hline $\mathrm{H}$ & 2935 & 3641 & 706 & & 4 (10 trials) \\
\hline I & 2418 & 3038 & 620 & & 10 (10 trials) \\
\hline J & 2674 & 3228 & 554 & & 6 (10 trials) \\
\hline $\mathrm{K}$ & 2776 & 4094.3 & 1318.3 & 6501.8 & c \\
\hline $\mathrm{L}$ & 2734 & 4049.6 & 1315.6 & 53287.2 & c \\
\hline M & 2676.6 & 4399.3 & 1722.7 & 30000 & 0 (15 trials) \\
\hline $\mathrm{N}$ & 2917.3 & 4158 & 1240.7 & 30000 & 0 (15 trials) \\
\hline 0 & 2766.3 & 4625 & 1858.7 & 7147.5 & 1 \\
\hline$P$ & 2826 & 4905 & 2079 & 6177.2 & 5 \\
\hline Q & 2615 & 4701 & 2086 & & 1 (10 trials) \\
\hline $\mathrm{R}$ & 2616 & 4136 & 1520 & & 2 (10 trials) \\
\hline$S$ & 3150 & 4543 & 1393 & & 2 (10 trials) \\
\hline $\mathrm{T}$ & 2620 & 5066 & 2446 & & 2 (10 trials) \\
\hline$U$ & 2564 & 4522 & 1958 & & 5 (10 trials) \\
\hline V & 2712 & 4618 & 1906 & & 5 (10 trials) \\
\hline W & 2921 & 4703.3 & 1782.3 & 25985.2 & c \\
\hline$x$ & 2758.6 & 5699.3 & 2940.7 & 30000 & 0 (15 trials) \\
\hline Y & 2772.6 & 4947.3 & 2174.7 & 30000 & 0 (15 trials) \\
\hline Z & 2846 & 5082.7 & 2236.7 & 30835.9 & 3 \\
\hline AA & 2686 & 4974.7 & 2288.7 & 29304.3 & c \\
\hline BB & 2771.7 & 4840.7 & 2069 & 30000 & 1 \\
\hline
\end{tabular}




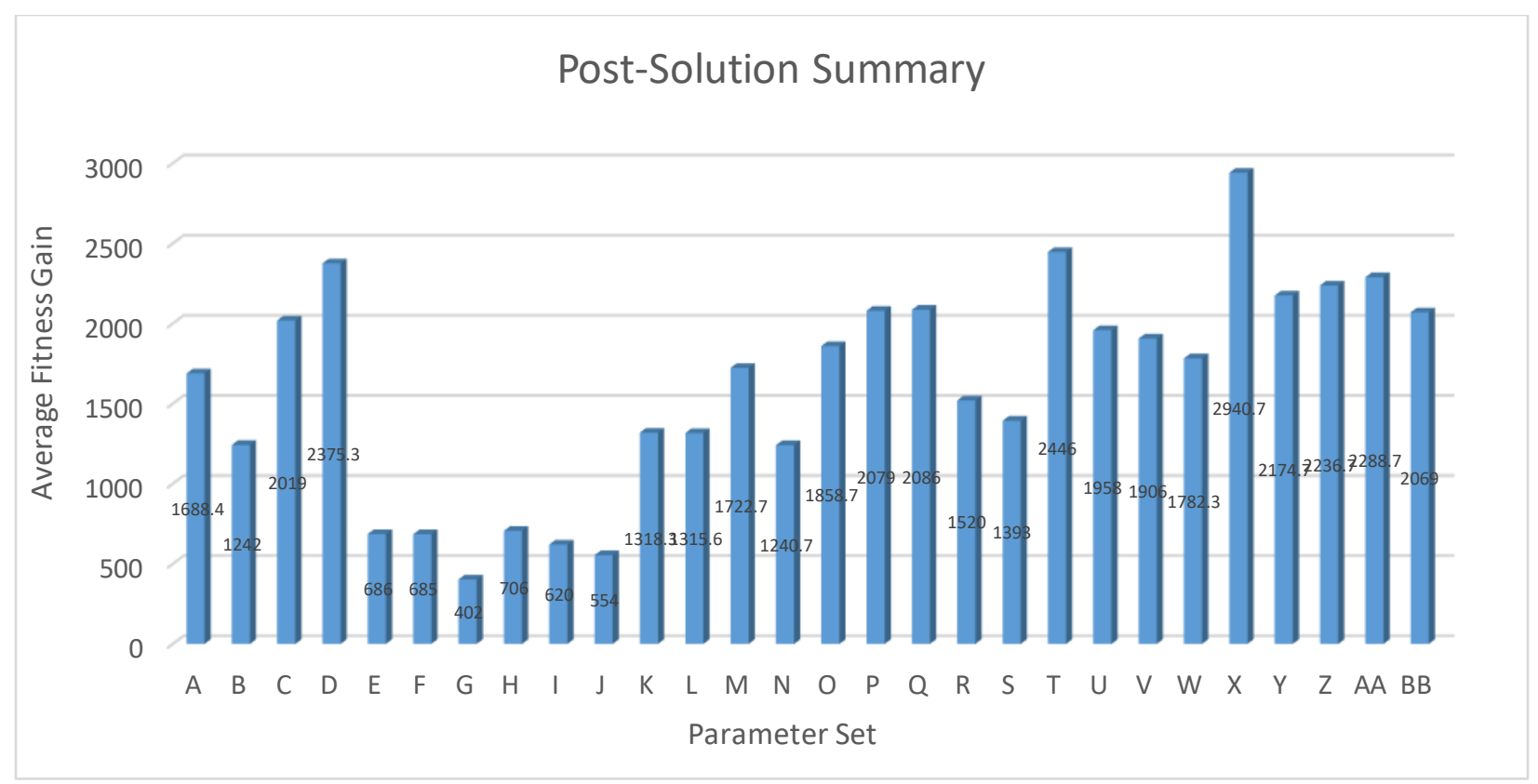




\subsubsection{Post-Solution Trials}
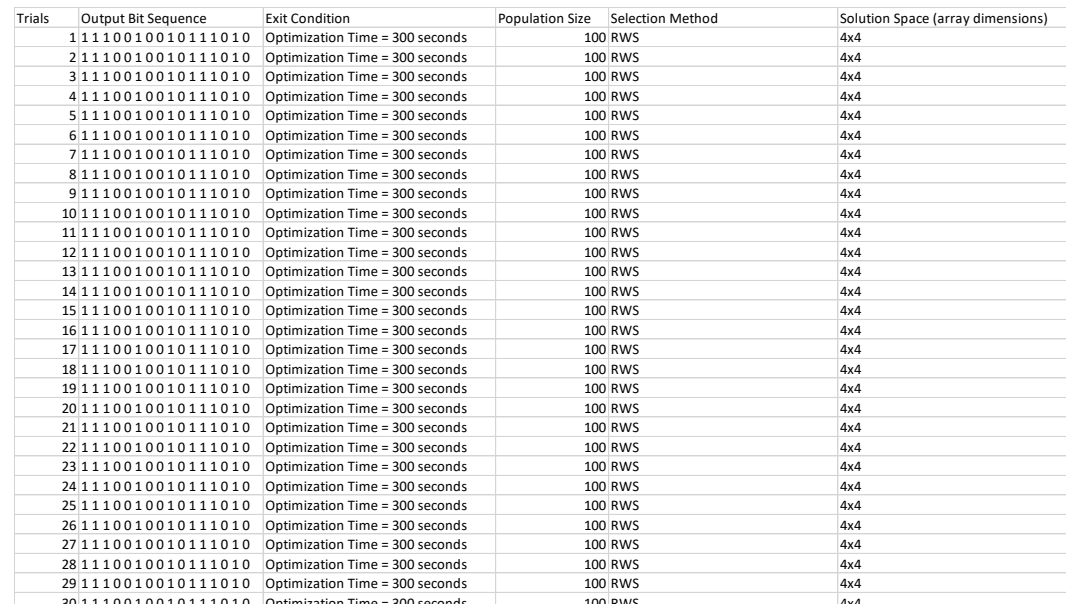

Starting Fitness

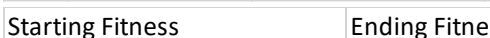

\begin{tabular}{|r|r|}
3850 \\
4010 \\
\hline 2610
\end{tabular}

$2610 \quad 4780$

$3130 \quad 5180$

$3260 \quad 4860$

$2410 \quad 4860$

$3420 \quad 5020$

$3250 \quad 4910$

$3580 \quad 4540$

$3260 \quad 4220$

$2700 \quad 4300$

$2610 \quad 3850$

$3020 \quad 4460$

$3420 \quad 4380$

$2410 \quad 5050$

$2970 \quad 3770$

$3260 \quad 5820$

$3660 \quad 4940$

$2410 \quad 4010$

$2860 \quad 4110$

$2300 \quad 5070$

$2300 \quad 4620$

$2460 \quad 4460$

$2450 \quad 4460$

LOST DATA IN POSTPROCESSING

\begin{tabular}{|r|r|r|r|}
\hline 4110 & 6460 & 35205 & 2350 \\
\hline 2300 & 3900 & 34052 & 1600 \\
\hline 2300 & 4060 & 24529 & 1760 \\
\hline 5714 & 4654.642857 & 25540.71429 & 1692.857143 \\
\hline
\end{tabular}

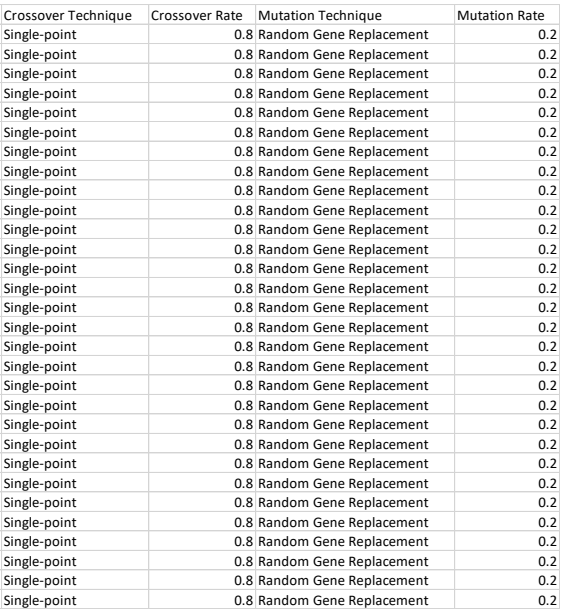

Difference

$$
26101
$$

23535

26395

33346

33126

23442

11947

11096

27064

27373

25979

22827

22700

23927

21902

23760

23581

24137

23864

22040

27579

26509

23869

32560

32695 1600

\# Trials Prematurely Converged 

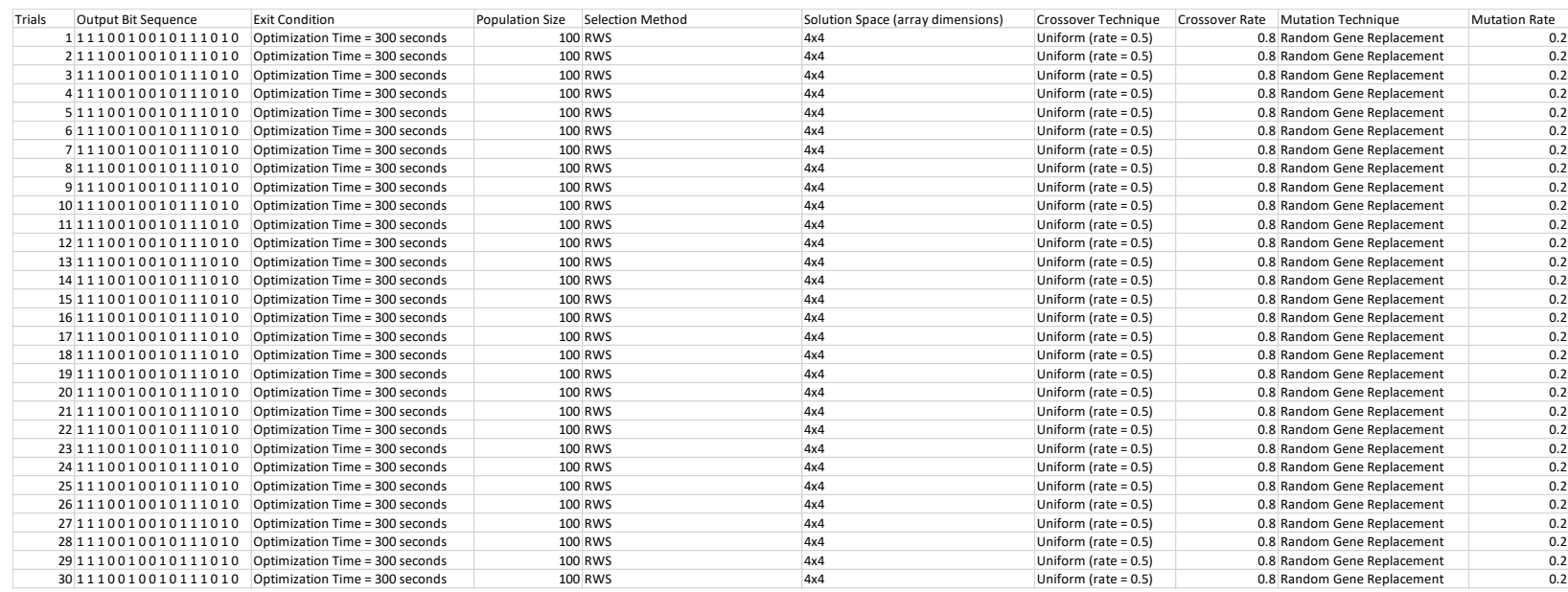

\begin{tabular}{|c|c|c|c|c|}
\hline Starting Fitness & Ending Fitness & Generations after solution found & Difference & \# Trials Prematurely Converge \\
\hline 2730 & 3210 & 22962 & 480 & 0 \\
\hline 3020 & 3210 & 24165 & 190 & \\
\hline 2460 & 3580 & 24218 & 1120 & \\
\hline 2700 & 3660 & 24647 & 960 & \\
\hline 2860 & 3100 & 24857 & 240 & \\
\hline 2410 & 5210 & 32567 & 2800 & \\
\hline 2350 & 3950 & 32568 & 1600 & \\
\hline 3260 & 4460 & 32556 & 1200 & \\
\hline 2700 & 4410 & 19494 & 1710 & \\
\hline 2770 & 3760 & 19671 & 990 & \\
\hline 3610 & 3660 & 18905 & 50 & \\
\hline 2700 & 4060 & 21988 & 1360 & \\
\hline 2510 & 4330 & 28242 & 1820 & \\
\hline 2860 & 4220 & 31647 & 1360 & \\
\hline 3210 & 4170 & 31927 & 960 & \\
\hline 4460 & 4460 & 30852 & 0 & \\
\hline 2300 & 4460 & 31516 & 2160 & \\
\hline 2810 & 4250 & 30737 & 1440 & \\
\hline 2460 & 4060 & 28877 & 1600 & \\
\hline 2620 & 3660 & 31304 & 1040 & \\
\hline 3260 & 4060 & 32156 & 800 & \\
\hline 2860 & 3500 & 33579 & 640 & \\
\hline 3260 & 5180 & 31433 & 1920 & \\
\hline 2770 & 4220 & 33280 & 1450 & \\
\hline 2610 & 3420 & 33495 & 810 & \\
\hline 2860 & 4060 & 33956 & 1200 & \\
\hline 2610 & 5020 & 34323 & 2410 & \\
\hline 2610 & 4220 & 31239 & 1610 & \\
\hline 3040 & 4940 & 32419 & 1900 & \\
\hline 3020 & 4460 & 32085 & 1440 & \\
\hline 2856.666667 & 4098.666667 & 29055.5 & 1242 & \\
\hline
\end{tabular}




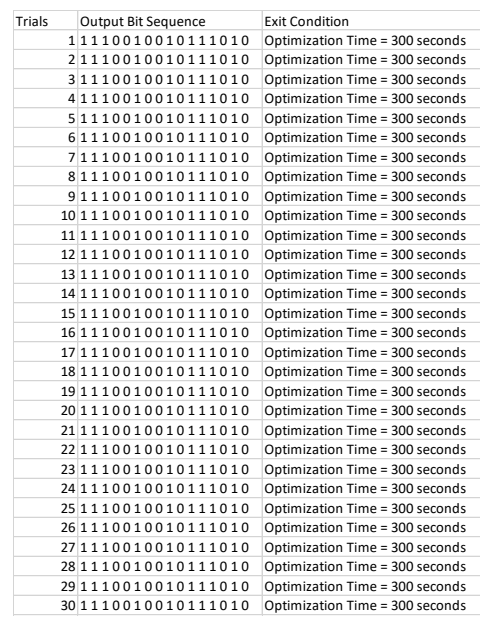

Population Size Selection Method

Selection Method 100 Tournament Selection - Size $=1$ 00 Tournament Selection - Size $=$ 100 Tournament Selection - Size $=1$ 100 Tournament Selection - Size $=$ 100 Tournament Selection - Size $=1$ 100 Tournament Selection - Size $=$ 100 Tournament Selection - Size $=1$ 100 Tournament Selection - Size $=1$ 100 Tournament Selection - Size $=1$ 100 Tournament Selection - Size $=1$ 100 Tournament Selection - Size $=1$ Tournament Selection - Size $=1$ 100 Tournament Selection - Size $=$ 100 Tournament Selection - Size $=1$ 100 Tournament Selection - Size $=$ 100 Tournament Selection - Size $=1$ 100 Tournament Selection - Size $=1$ 100 Tournament Selection - Size $=1$ 100 Tournament Selection - Size $=1$ 100 Tournament Selection - Size $=1$ 100 Tournament Selection - Size $=1$ 100 Tournament Selection - Size $=$ 100 Tournament Selection - Size $=1$ 100 Tournament Selection - Size $=$ 100 Tournament Selection - Size $=$

Crossover Technique Crossover Rate Mutation Technique 0.8 Random Gene Replacement 0.8 Random Gene Replacement 8 Random Gene Replacement 0.8 Random Gene Replacement 0.8 Random Gene Replacement 0.8 Random Gene Replacement 8 Random Gene Replacement 0.8 Random Gene Replacement 0.8 Random Gene Replacement 8 Random Gene Replacement 8 Random Gene Replacement 8 Random Gene Replacement 8 Random Gene Replacement 8 Random Gene Replacement 0.8 Random Gene Replacement 8 Random Gene Replacement 0.8 Random Gene Replacement 0.8 Random Gene Replacement 0.8 Random Gene Replacement 8 Random Gene Replacement 0.8 Random Gene Replacement 8 Random Gene Replacement 8 Random Gene Replacement 0.8 Random Gene Replacement 0.8 Random Gene Replacement

0.8 Random Gene Replacement

Mutation Rate

\begin{tabular}{|c|c|c|c|c|}
\hline Starting Fitness & Ending Fitness & Generations after solution found & Difference & \# Trials Prematurely Converge \\
\hline 2620 & 5420 & 30751 & 2800 & 7 \\
\hline 3260 & 6620 & 32579 & 3360 & \\
\hline 2860 & 4860 & 30303 & 2000 & \\
\hline 3260 & 5020 & 31688 & 1760 & \\
\hline 2700 & 4060 & 31820 & 1360 & \\
\hline 2850 & 4970 & 31907 & 2120 & \\
\hline 3100 & 6620 & 33676 & 3520 & \\
\hline 2300 & 4220 & 32669 & 1920 & \\
\hline 2700 & 4380 & 25402 & 1680 & \\
\hline 3420 & 4540 & 29154 & 1120 & \\
\hline 2860 & 5020 & 29876 & 2160 & \\
\hline 4060 & 5020 & 30191 & 960 & \\
\hline 2450 & 4380 & 30256 & 1930 & \\
\hline 2300 & 4700 & 23621 & 2400 & \\
\hline 3260 & 4860 & 17054 & 1600 & \\
\hline 2300 & 4940 & 20033 & 2640 & \\
\hline 2570 & 4410 & 15751 & 1840 & \\
\hline 2700 & 3900 & 17150 & 1200 & \\
\hline 3100 & 4110 & 33591 & 1010 & \\
\hline 2460 & 5980 & 35446 & 3520 & \\
\hline 2780 & 4570 & 34641 & 1790 & \\
\hline 2300 & 4780 & 35298 & 2480 & \\
\hline 2300 & 3260 & 34308 & 960 & \\
\hline 2300 & 4540 & 34545 & 2240 & \\
\hline 2460 & 5210 & 34240 & 2750 & \\
\hline 2450 & 6300 & 35809 & 3850 & \\
\hline 3100 & 4060 & 34983 & 960 & \\
\hline 2460 & 4700 & 27072 & 2240 & \\
\hline 2460 & 3260 & 31805 & 800 & \\
\hline 3260 & 4860 & 26587 & 1600 & \\
\hline 2766.666667 & 4785.666667 & 29740.2 & 2019 & \\
\hline
\end{tabular}




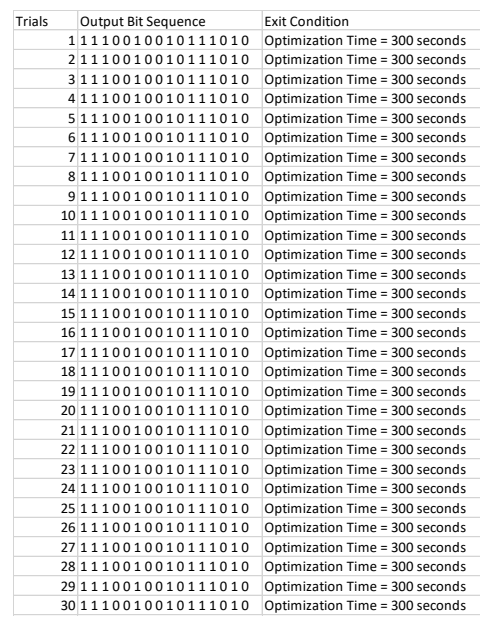

Population Size Selection Method 100 RWS 100 RWS 100 RWS 100 RWS 100 RWS 100 RWS 100 RWS 100 RWS 100 RWS 100 RWS 100 RWS 100 RWS 100 RWS 100 RWS 100 RWS 100 RWS 100 RWS 100 RWS 100 RWS 100 RWS 100 RWS 100 RWS 100 RWS 100 RWS 100 RWS 100 RWS

$$
\text { Generation }
$$

Generations after solution found

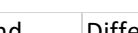

Difference

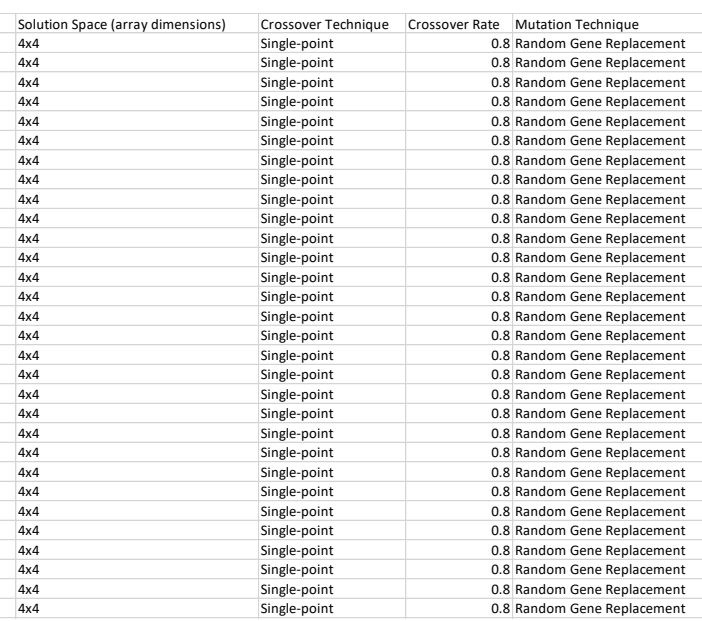

Mutation Rate

Starting Fitness

\begin{tabular}{|c|c|c|c|c|}
\hline & Ending Fitness & Generations after solution found & Difference & \# Trials Prematurely Converge \\
\hline 3100 & 4810 & 30119 & 1710 & 5 \\
\hline 2620 & 5130 & 30933 & 2510 & \\
\hline 2300 & 5150 & 33003 & 2850 & \\
\hline 2450 & 5290 & 30298 & 2840 & \\
\hline 2460 & 5420 & 30592 & 2960 & \\
\hline 2620 & 5370 & 27525 & 2750 & \\
\hline 2860 & 5210 & 28358 & 2350 & \\
\hline 3260 & 4780 & 34662 & 1520 & \\
\hline 2620 & 5610 & 25446 & 2990 & \\
\hline 3820 & 5020 & 26288 & 1200 & \\
\hline 2460 & 4510 & 29429 & 2050 & \\
\hline 3660 & 5100 & 34087 & 1440 & \\
\hline 2860 & 4620 & 34002 & 1760 & \\
\hline 2510 & 5310 & 34101 & 2800 & \\
\hline 2460 & 4810 & 32865 & 2350 & \\
\hline 2460 & 5420 & 34127 & 2960 & \\
\hline 2450 & 5550 & 34459 & 3100 & \\
\hline 2300 & 5020 & 30251 & 2720 & \\
\hline 2700 & 6170 & 30541 & 3470 & \\
\hline 3250 & 5740 & 30113 & 2490 & \\
\hline 2700 & 6570 & 28960 & 3870 & \\
\hline 3100 & 4700 & 29778 & 1600 & \\
\hline 2860 & 4270 & 26138 & 1410 & \\
\hline 2460 & 5020 & 25295 & 2560 & \\
\hline 2400 & 5500 & 37451 & 3100 & \\
\hline 3260 & 5020 & 35967 & 1760 & \\
\hline 2460 & 4780 & 35977 & 2320 & \\
\hline 3260 & 5610 & 36101 & 2350 & \\
\hline 3900 & 6410 & 35999 & 2510 & \\
\hline 3500 & 4460 & 36086 & 960 & \\
\hline 33333 & 5212.666667 & 31631.7 & 2375.333333 & \\
\hline
\end{tabular}




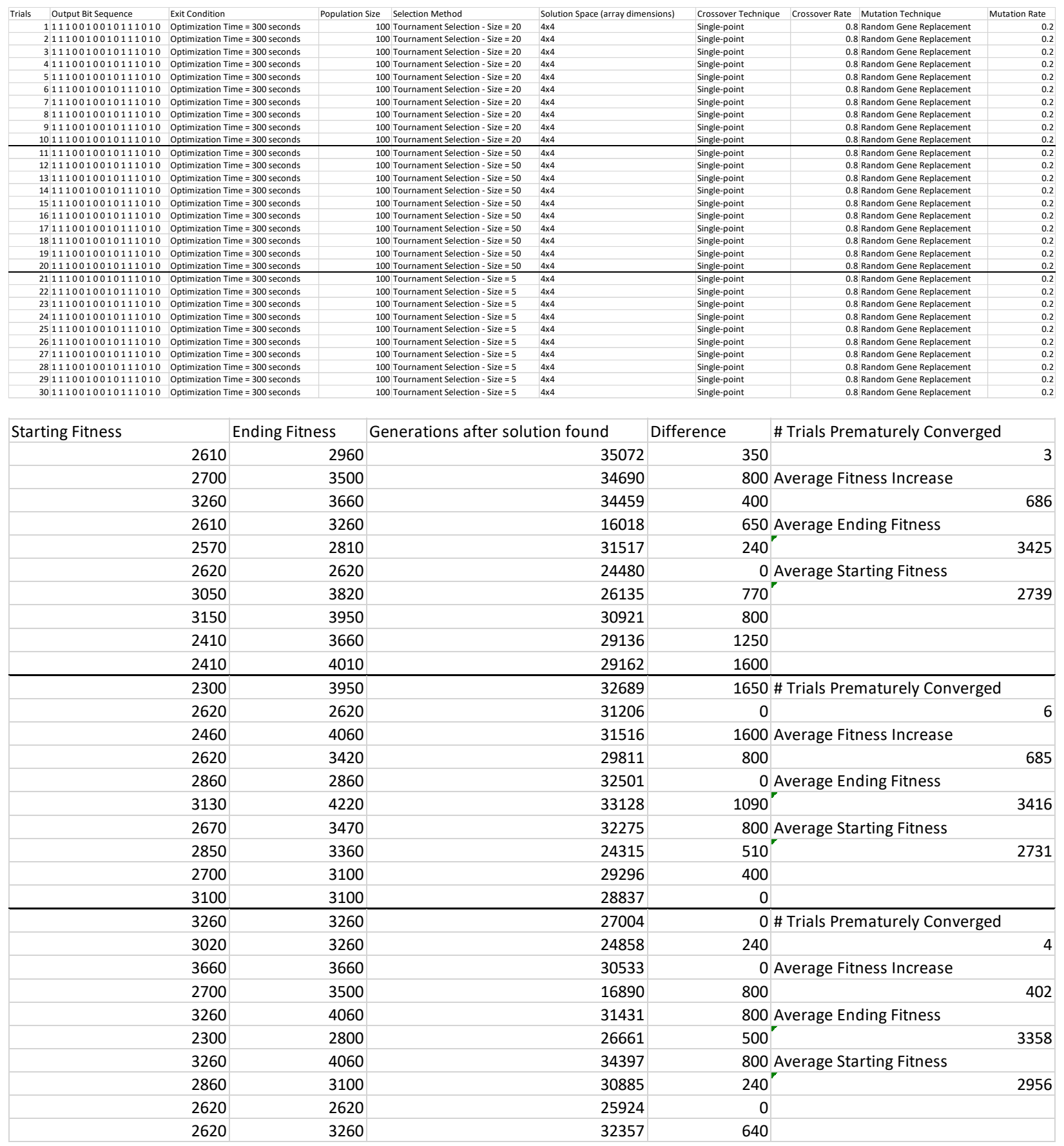




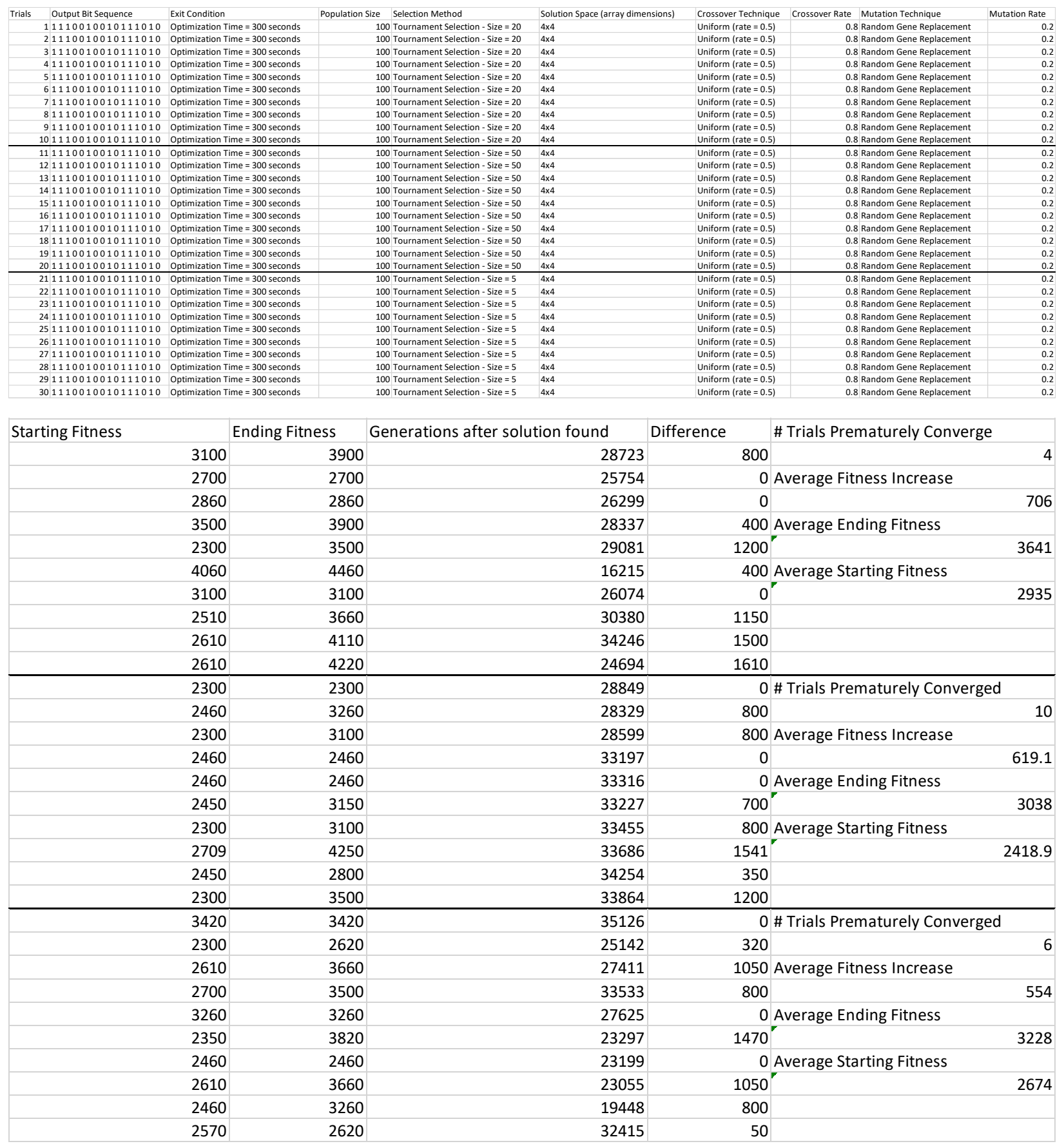




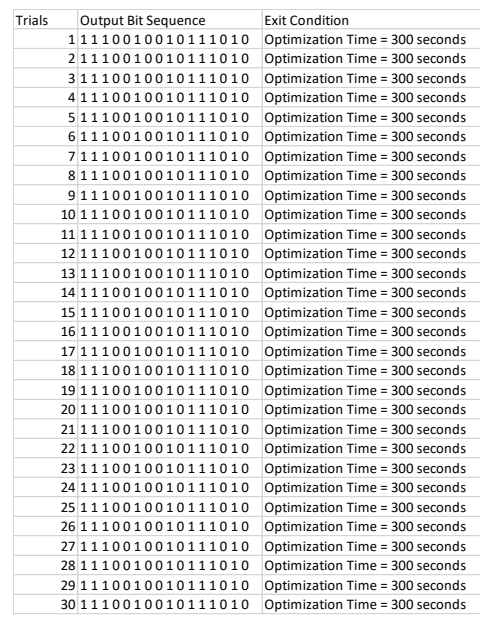

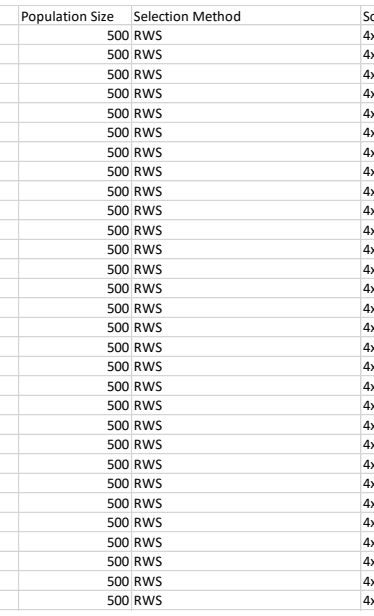
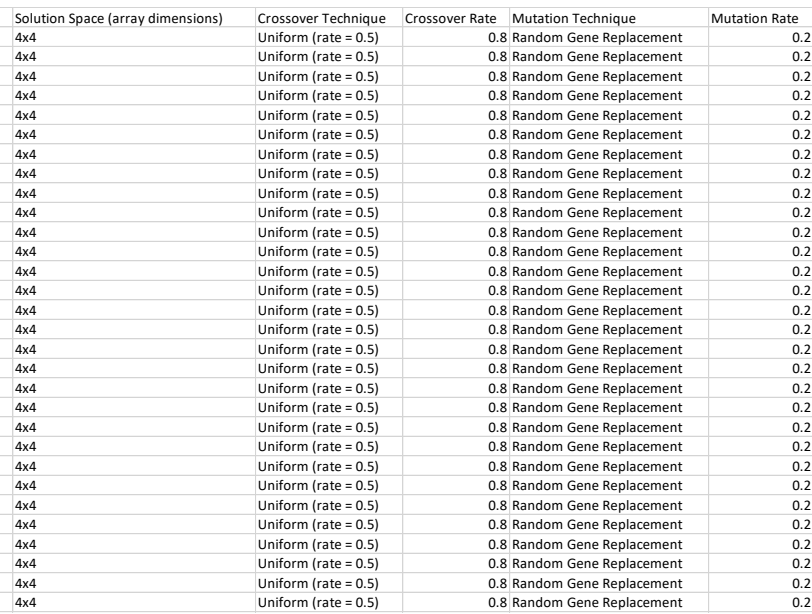

Starting Fitness

\begin{tabular}{|c|c|c|c|c|}
\hline & Ending Fitness & Generations after solution found & Difference & \# Trials Prematurely Converged \\
\hline 2780 & 2940 & 7039 & 160 & 0 \\
\hline 2620 & 4060 & 7151 & 1440 & \\
\hline 2860 & 3660 & 7289 & 800 & \\
\hline 2620 & 3420 & 5211 & 800 & \\
\hline 2800 & 4060 & 5972 & 1260 & \\
\hline 3370 & 4380 & 6108 & 1010 & \\
\hline 3820 & 5020 & 6950 & 1200 & \\
\hline 2960 & 4330 & 6253 & 1370 & \\
\hline 2450 & 4860 & 6576 & 2410 & \\
\hline 3500 & 3900 & 6449 & 400 & \\
\hline 2930 & 4430 & 7498 & 1500 & \\
\hline 2610 & 4110 & 7462 & 1500 & \\
\hline 2450 & 3530 & 7532 & 1080 & \\
\hline 2960 & 4060 & 7451 & 1100 & \\
\hline 2450 & 4910 & 7483 & 2460 & \\
\hline 2410 & 3470 & 7368 & 1060 & \\
\hline 3660 & 3900 & 7335 & 240 & \\
\hline 2450 & 4110 & 7500 & 1660 & \\
\hline 2460 & 3260 & 7082 & 800 & \\
\hline 3420 & 4460 & 7086 & 1040 & \\
\hline 2450 & 4060 & 7271 & 1610 & \\
\hline 2410 & 4810 & 7029 & 2400 & \\
\hline 2730 & 2970 & 2450 & 240 & \\
\hline 2450 & 4620 & 7077 & 2170 & \\
\hline 2610 & 4620 & 5168 & 2010 & \\
\hline 2560 & 4700 & 5070 & 2140 & \\
\hline 2610 & 3950 & 5065 & 1340 & \\
\hline 2560 & 4110 & 5007 & 1550 & \\
\hline 2460 & 4060 & 6012 & 1600 & \\
\hline 2860 & 4060 & 6110 & 1200 & \\
\hline 2776 & 4094.333333 & 6501.8 & 1318.333333 & \\
\hline
\end{tabular}



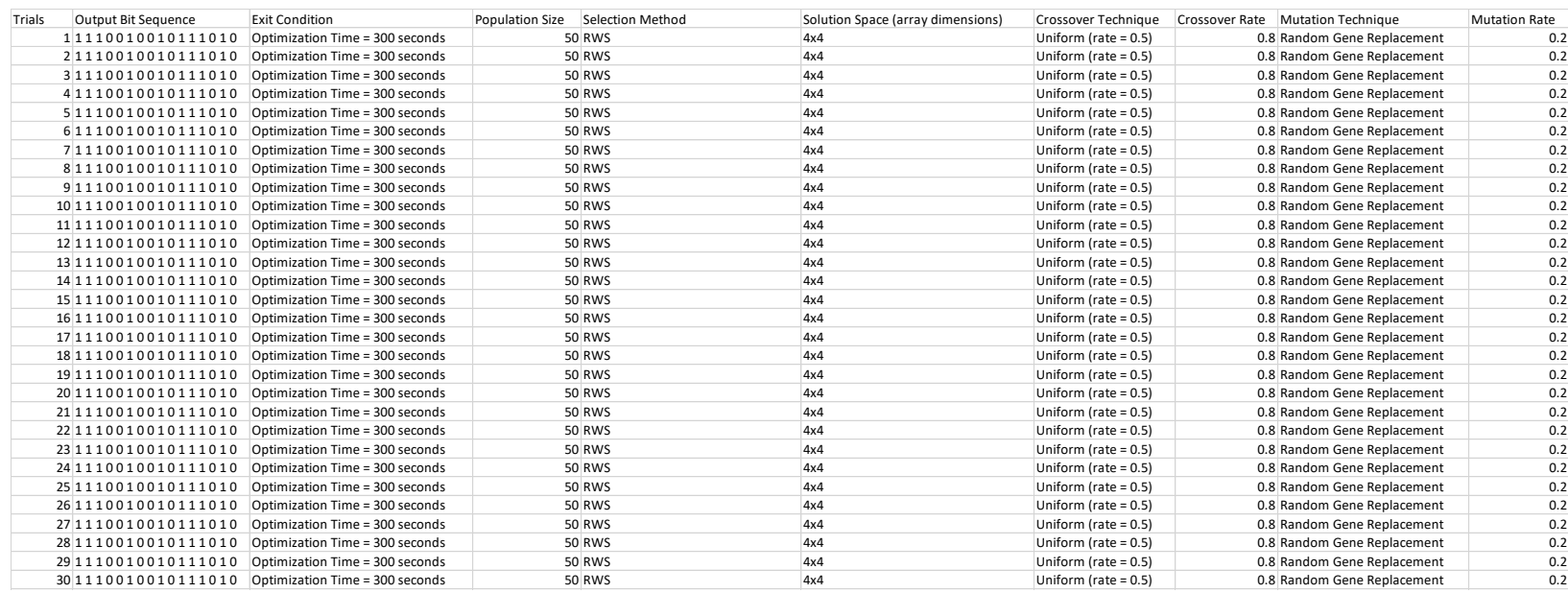

\begin{tabular}{|c|c|c|c|c|}
\hline Starting Fitness & Ending Fitness & Generations after solution found & Difference & \# Trials Prematurely Converged \\
\hline 2730 & 4570 & 57049 & 1840 & 0 \\
\hline 2460 & 4410 & 62019 & 1950 & \\
\hline 2300 & 3260 & 62340 & 960 & \\
\hline 2400 & 3900 & 62433 & 1500 & \\
\hline 3020 & 4380 & 61393 & 1360 & \\
\hline 3420 & 4060 & 62963 & 640 & \\
\hline 2300 & 4250 & 61744 & 1950 & \\
\hline 3370 & 4300 & 62498 & 930 & \\
\hline 2300 & 3900 & 63584 & 1600 & \\
\hline 2620 & 4700 & 62766 & 2080 & \\
\hline 3260 & 3260 & 62011 & 0 & \\
\hline 2460 & 4270 & 59495 & 1810 & \\
\hline 2650 & 4810 & 55505 & 2160 & \\
\hline 2300 & 5020 & 55947 & 2720 & \\
\hline 2460 & 4060 & 31374 & 1600 & \\
\hline 2610 & 3260 & 33615 & 650 & \\
\hline 3020 & 4300 & 25947 & 1280 & \\
\hline 3500 & 3500 & 48716 & 0 & \\
\hline 3020 & 5260 & 50655 & 2240 & \\
\hline 2300 & 3660 & 46345 & 1360 & \\
\hline 2890 & 3260 & 50134 & 370 & \\
\hline 2620 & 3420 & 52405 & 800 & \\
\hline 2610 & 5180 & 55885 & 2570 & \\
\hline 2300 & 4300 & 51379 & 2000 & \\
\hline 2730 & 4220 & 48138 & 1490 & \\
\hline 2610 & 3420 & 50011 & 810 & \\
\hline 2860 & 4220 & 55283 & 1360 & \\
\hline 2300 & 3260 & 47416 & 960 & \\
\hline 3980 & 4060 & 52921 & 80 & \\
\hline 2620 & 3020 & 46644 & 400 & \\
\hline 2734 & 4049.666667 & 53287.16667 & 1315.666667 & \\
\hline
\end{tabular}




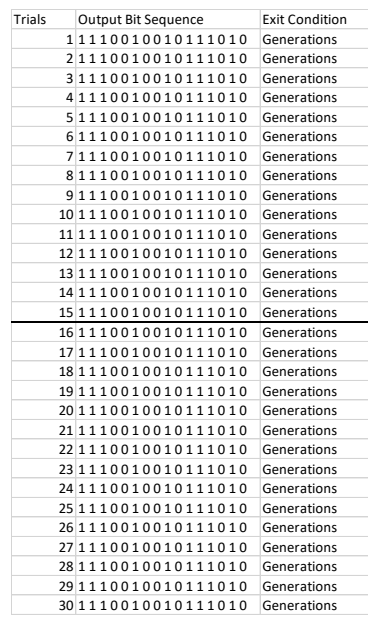

Population Size Selection Method 500 RWS 500 RWS 500 RWS 500 RWS 500 RWS 500 RWS 500 RWS 500 RWS 500 RWS 00 RWS 500 RWS 500 RWS 500 RWS 500 RWS 50 RWS 50 RWS 50 RWS 50 RWS 50 RWS

50 RWS

RO RWS

50 RWS

50 RWS

50 RWS

50 RWS

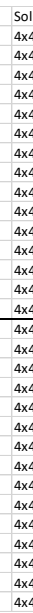

Solut
$4 \times 4$
$4 \times 4$
$4 \times 4$
$4 \times 4$
$4 \times 4$
$4 \times 4$
$4 \times 4$
$4 \times 4$
$4 \times 4$
$4 \times 4$
$4 \times 4$
$4 \times 4$
$4 \times 4$
$4 \times 4$
$4 \times 4$
$4 \times 4$
$4 \times 4$
$4 \times 4$
$4 \times 4$
$4 \times 4$
$4 \times 4$
$4 \times 4$
$4 \times 4$
$4 \times 4$
$4 \times 4$
$4 \times 4$
$4 \times 4$
$4 \times 4$
$4 \times 4$
$4 \times 4$ Uniform (rate $=0.5$ ) Uniform (rate $=0.5)$

Uniform (rate $=0.5)$

Uniform (rate $=0.5$ )

Uniform (rate $=0.5$ )

niform (rate $=0.5$ )

Uniform (rate $=0.5$ )

Uniform (rate $=0.5$ )
Uniform (rate $=0.5$ )

niform (rate $=0.5$ )

Uniform (rate $=0.5$ )

Uniform (rate $=0.5$ )

Un

Uniform (rate $=0.5$ )

niform (rate $=0.5$ )

Uniform (rate $=0.5$ )

Uniform (rate $=0.5$ )

Uniform (rate $=0.5$ )

Starting Fitness

Ending Fitness Generations after solution found

Difference $30000 \quad$ Difference

$30000 \quad 1360$

30000

$30000 \quad 1310$

$30000 \quad 850$

$30000 \quad 1660$

$30000 \quad 1600$

$30000 \quad 1500$

$30000 \quad 1660$

$30000 \quad 1360$

$30000 \quad 2120$

$30000 \quad 2690$

$30000 \quad 1280$

$30000 \quad 2250$

$30000 \quad 2540$

$\begin{array}{ll}30000 & 1810 \\ 30000 & 2560\end{array}$

$\begin{array}{rr}30000 & 2560 \\ 30000 & 800\end{array}$

30000

30000

30000

30000

30000

30000

30000

30000

30000

30000

30000

\begin{tabular}{r|r|r|}
\hline 4860 & 30000 & 1760 \\
\hline 4860 & 30000 & 560
\end{tabular}

$\begin{array}{rrr}4860 & 30000 & 1760 \\ 4860 & 30000 & 560\end{array}$
Computation Time

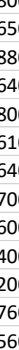

4860

285.086
$560 \quad 278.338$

te Mutation Technique 0.8 Random Gene Replacement 0.8 Random Gene Replacement 0.8 Random Gene Replacement 0.8 Random Gene Replacement 0.8 Random Gene Replacement 0.8 Random Gene Replacement 0.8 Random Gene Replacement 0.8 Random Gene Replacement 0.8 Random Gene Replacement 08 Rand 08 Pandom Gene Replacement 0.8 Random Gene Replacement andom Gene Replacement . Random Gene Replacement 0.8 Random Gene Replacement 0.8 Random Gene Replacement 0.8 Random Gene Replacement 0.8 Random Gene Replacement 0.8 Random Gene Replacement 08 Random Gene Replacement 08 Random Gene Replacement 0.8 Random Gene Replacement

0.8 Random Gene Replacement 0.8 Random Gene Replacement 0.8 Random Gene Replacement

Mutation Rate 0.2 0.2
0.2
0.2
0.2
0.2
0.2
0.2
0.2
0.2
0.2
0.2
0.2
0.2
0.2
0.2
0.2
0.2
0.2
0.2
0.2
0.2
0.2
0.2
0.2
0.2
0.2
0.2
0.2
0.2
0.2

rials Prematurely Converged 1600.793 \#27.089 Average Fitness Gain 1257.764 1276.846 Average Ending Fitness $1246.659^{\prime \prime}$

1242.015 Average Starting Fitness $1174.86^{\prime \prime}$ 1170.453 Average Computation Time $1163.663^{\prime \prime}$

1173.703

2451.653

1403.505

1384.282

1327.504 1330.671

295.448 \# Trials Prematurely Converged 328.398

292.494 Average Fitness Gain

292.708

285.412 Average Ending Fitness

$286.717^{\prime \prime}$

291.381

288.871"

281.588

288.841

282.657

285.591

285.086

278.338

1722.666667

4399.333333

676.666667

1382.097333

(2)

$+2$

ged

1240.666667

4158

2917.333333

290.3625333 


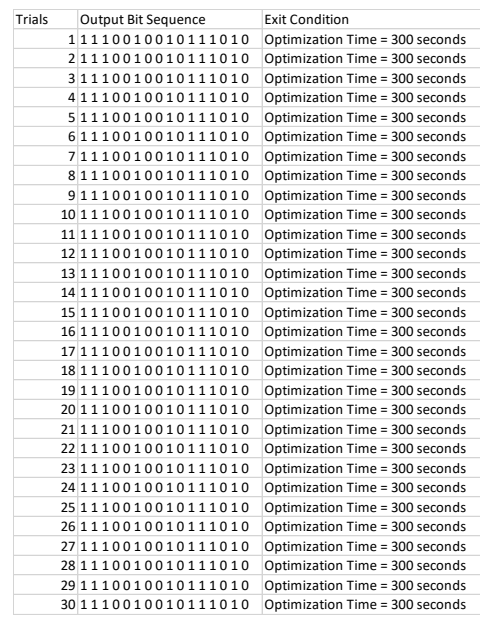

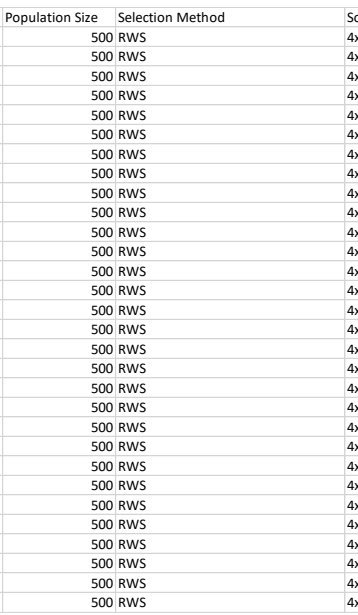
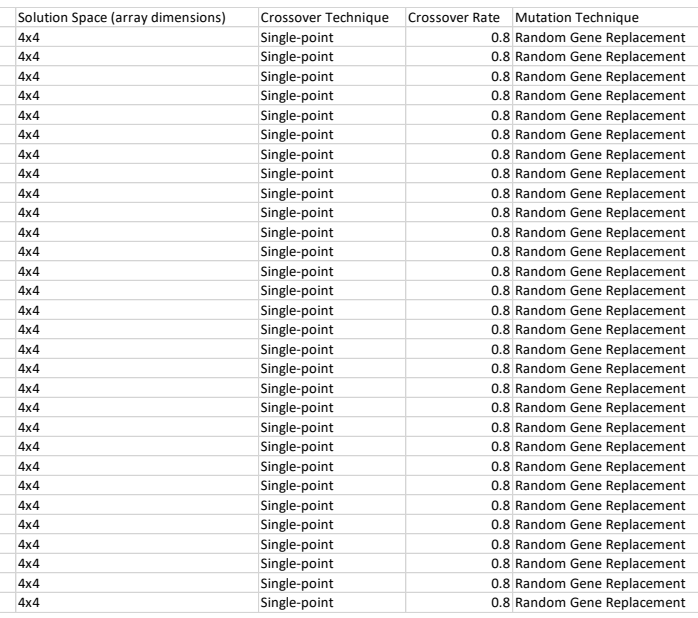

Mutation Rate 1110010010111010 Optimization Time $=300$ seconds

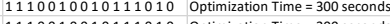
301110010010111010 Optimization Time $=300$ seconds

Starting Fitness

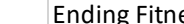

\begin{tabular}{|c|c|c|c|c|}
\hline Starting Fitness & Ending Fitness & Generations after solution found & Difference & \# Trials Prematurely Converged \\
\hline 2860 & 5020 & 7118 & 2160 & 1 \\
\hline 2450 & 4860 & 7559 & 2410 & \\
\hline 2560 & 4860 & 7033 & 2300 & \\
\hline 2410 & 3610 & 7090 & 1200 & \\
\hline 2750 & 5020 & 7293 & 2270 & \\
\hline 2460 & 5020 & 7214 & 2560 & \\
\hline 2810 & 4810 & 7225 & 2000 & \\
\hline 3100 & 4300 & 6673 & 1200 & \\
\hline 2460 & 4380 & 7502 & 1920 & \\
\hline 2450 & 3710 & 7482 & 1260 & \\
\hline 3850 & 4730 & 7488 & 880 & \\
\hline 3260 & 4060 & 7472 & 800 & \\
\hline 2450 & 4110 & 7523 & 1660 & \\
\hline 2250 & 4010 & 7368 & 1760 & \\
\hline 2460 & 4620 & 7494 & 2160 & \\
\hline 3020 & 5420 & 5240 & 2400 & \\
\hline 2730 & 5180 & 5448 & 2450 & \\
\hline 3260 & 4570 & 6366 & 1310 & \\
\hline 3660 & 6060 & 6598 & 2400 & \\
\hline 2410 & 3610 & 6948 & 1200 & \\
\hline 3100 & 5610 & 7414 & 2510 & \\
\hline 2300 & 4220 & 7213 & 1920 & \\
\hline 2620 & 3660 & 7382 & 1040 & \\
\hline 2970 & 4490 & 7415 & 1520 & \\
\hline 2460 & 4860 & 7559 & 2400 & \\
\hline 2300 & 5500 & 7450 & 3200 & \\
\hline 2450 & 3950 & 7432 & 1500 & \\
\hline 2610 & 5340 & 7595 & 2730 & \\
\hline 3260 & 4060 & 7363 & 800 & \\
\hline 3260 & 5100 & 7468 & 1840 & \\
\hline 2766.333333 & 4625 & 7147.5 & 1858.666667 & \\
\hline
\end{tabular}

Difference




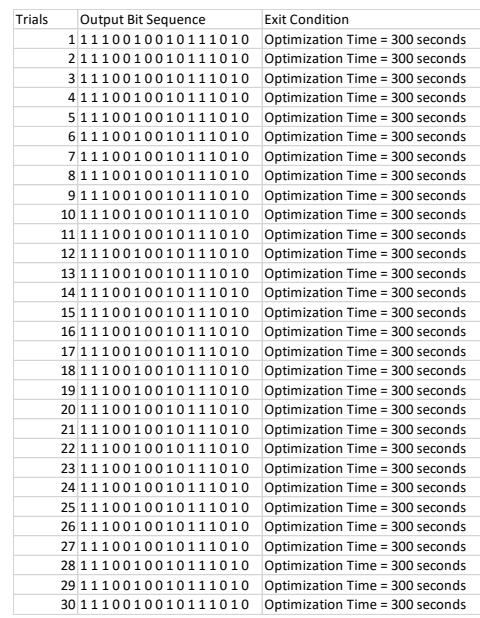

Population Size Selection Method

thod 500 Tournament Selection - Size $=1$ Do Tournament Selection - Size $=$ nt Selection - Size $=1$ 500 Tournament Selection - Size $=$ Tounament Selection - Size $=1$ 500 Tournament Selection - Size $=$ 500 Tournament Selection - Size $=1$ 500 Tournament Selection - Size $=1$ 500 Tournament Selection - Size $=1$ 500 Tournament Selection - Size $=1$ 500 Tournament Selection - Size $=1$ 500 Tournament Selection - Size $=$ 500 Tournament Selection - Size $=1$ 500 Tournament Selection - Size $=1$ 500 Tournament Selection - Size $=1$ 500 Tournament Selection - Size $=1$ 500 Tournament Selection - Size $=1$ 500 Tournament Selection - Size $=1$ 500 Tournament Selection - Size $=1$ 500 Tournament Selection - Size $=1$ 500 Tournament Selection - Size $=$ 500 Tournament Selection - Size $=1$ 500 Tournament Selection - Size $=1$ 500 Tournament Selection - Size $=1$
500 Tournament Selection - Size $=1$
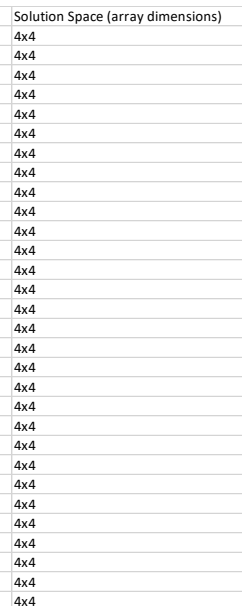
\begin{tabular}{l} 
Crossover Te \\
Single-point \\
Single-point \\
Single-point \\
Single-point \\
Single-point \\
Single-point \\
Single-point \\
Single-point \\
Single-point \\
Single-point \\
Single-point \\
\hline Single-point \\
Single-point \\
Single-point \\
Single-point \\
\hline Single-point \\
Single-point \\
Single-point \\
Single-point \\
Single-point \\
Single-point \\
Single-point \\
Single-point \\
Single-point \\
Single-point \\
Single-point \\
Single-point \\
\hline Single-point \\
Single-point \\
Single-point
\end{tabular} Crossover Rate Mutation Technique 0.8 Random Gene Replacement 0.8 Random Gene Replacement 0.8 Random Gene Replacement 0.8 Random Gene Replacement 0.8 Random Gene Replacement 0.8 Random Gene Replacement 0.8 Random Gene Replacement 0.8 Random Gene Replacement 08 Random Gene Replacement 0.8 Random Gene Replacement .8.8 Random Gene 8 Random Gene Replacement 8 Random Gene Replacement 0.8 Random Gene Replacement 8 Random Gene Replacement 0.8 Random Gene Replacement 88 Random Gene Replacement 0.8 Random Gene Replacement 0.8 Random Gene Replacement 8 Random Gene Replacement 8 Random Gene Replacement 88 Random Gene Replacement 8 Random Gene Replacement 8 Random Gene Replacement 0.8 Random Gene Replacement 0.8 Random Gene Replacement 0.8 Random Gene Replacement

Mutation Rate

\begin{tabular}{|c|c|c|c|c|}
\hline Starting Fitness & Ending Fitness & Generations after solution found & Difference & \# Trials Prematurely Converged \\
\hline 4250 & 4730 & 6797 & 480 & 5 \\
\hline 2810 & 4410 & 6736 & 1600 & \\
\hline 2780 & 4620 & 6999 & 1840 & \\
\hline 2700 & 5020 & 7254 & 2320 & \\
\hline 2300 & 5290 & 4342 & 2990 & \\
\hline 2300 & 4060 & 3989 & 1760 & \\
\hline 2450 & 4270 & 4249 & 1820 & \\
\hline 2300 & 5260 & 4243 & 2960 & \\
\hline 2860 & 4730 & 2854 & 1870 & \\
\hline 2700 & 4460 & 4892 & 1760 & \\
\hline 2780 & 6620 & 5841 & 3840 & \\
\hline 2860 & 5020 & 7513 & 2160 & \\
\hline 3210 & 5180 & 7285 & 1970 & \\
\hline 2620 & 5290 & 6857 & 2670 & \\
\hline 2460 & 4220 & 5554 & 1760 & \\
\hline 4010 & 6570 & 5836 & 2560 & \\
\hline 2300 & 4460 & 7181 & 2160 & \\
\hline 3420 & 4620 & 5757 & 1200 & \\
\hline 2780 & 4460 & 7638 & 1680 & \\
\hline 2620 & 4170 & 5263 & 1550 & \\
\hline 2300 & 4380 & 7186 & 2080 & \\
\hline 2850 & 5530 & 6570 & 2680 & \\
\hline 3360 & 5660 & 5361 & 2300 & \\
\hline 2610 & 5870 & 7471 & 3260 & \\
\hline 2860 & 3820 & 6858 & 960 & \\
\hline 3370 & 6010 & 6977 & 2640 & \\
\hline 2300 & 4110 & 6751 & 1810 & \\
\hline 2610 & 4510 & 7040 & 1900 & \\
\hline 3020 & 4060 & 7069 & 1040 & \\
\hline 3010 & 5740 & 6953 & 2730 & \\
\hline 2826.666667 & 4905 & 6177.2 & 2078.333333 & \\
\hline
\end{tabular}




\begin{tabular}{|c|c|c|c|c|c|c|c|c|}
\hline Trials Output Bit Sequence & Exit Condition & & Population Size & ze Selection Method & Solution Space (array dimensio & Crossover Technique & Crossover Rate Mutation Technique & Mutation Rate \\
\hline 11110010010111010 & Optimization Tim & ime $=1000$ seconds & & 100 RWS & $4 \times 4$ & Single-point & 0.8 Random Gene Replacement & 0.2 \\
\hline 211100100101110100 & Optimization Tim & ime $=1000$ seconds & & 100 RWS & $4 \times 4$ & Single-point & 0.8 Random Gene Replacement & 0.2 \\
\hline 311100100101110100 & Optimization Tim & ime = 1000 seconds & & 100 RWS & $4 \times 4$ & Single-point & 0.8 Random Gene Replacement & 0.2 \\
\hline 411100100101110100 & Optimization Tim & ime $=1000$ seconds & & 100 RWS & $4 \times 4$ & Single-point & 0.8 Random Gene Replacement & 0.2 \\
\hline 51110010010111010 & Optimization Tim & ime $=1000$ seconds & & 100 RWS & $4 \times 4$ & Single-point & 0.8 Random Gene Replacement & 0.2 \\
\hline 611100100101110100 & Optimization Tim & ime $=1000$ seconds & & 100 RWS & $4 \times 4$ & Single-point & 0.8 Random Gene Replacement & 0.2 \\
\hline 711100100101110100 & Optimization Tim & ime $=1000$ seconds & & 100 RWS & $4 \times 4$ & Single-point & 0.8 Random Gene Replacement & 0.2 \\
\hline 811100100101110100 & Optimization Tim & ime $=1000$ seconds & & 100 RWS & $4 \times 4$ & Single-point & 0.8 Random Gene Replacement & 0.2 \\
\hline 911100100101110100 & Optimization Tim & ime $=1000$ seconds & & 100 RWS & $4 \times 4$ & Single-point & 0.8 Random Gene Replacement & 0.2 \\
\hline 1011100100101110100 & Optimization Tim & ime $=1000$ seconds & & 100 RWS & $4 \times 4$ & Single-point & 0.8 Random Gene Replacement & 0.2 \\
\hline 111110010010111010 & Optimization Tim & ime $=100$ seconds & & 100 RWS & $\frac{4 \times 4}{4 \times 4}$ & Single-point & 0.8 Random Gene Replacement & 0.2 \\
\hline 1211100100101110100 & Optimization Tim & ime $=100$ seconds & & 100 RWS & $4 \times 4$ & Single-point & 0.8 Random Gene Replacement & 0.2 \\
\hline 131110010010111010 & Optimization Tim & ime $=100$ seconds & & 100 RWS & $4 \times 4$ & Single-point & 0.8 Random Gene Replacement & 0.2 \\
\hline 1411100100101110100 & Optimization Tim & ime $=100$ seconds & & 100 RWS & $4 \times 4$ & Single-point & 0.8 Random Gene Replacement & 0.2 \\
\hline 1511100100101110100 & Optimization Tim & ime $=100$ seconds & & 100 RWS & $4 \times 4$ & Single-point & 0.8 Random Gene Replacement & 0.2 \\
\hline 161110010010111010 & Optimization Tim & ime $=100$ seconds & & 100 RWS & $4 \times 4$ & Single-point & 0.8 Random Gene Replacement & 0.2 \\
\hline 171110010010111010 & Optimization Tim & $\mathrm{ime}=100$ seconds & & 100 RWS & $4 \times 4$ & Single-point & 0.8 Random Gene Replacement & 0.2 \\
\hline 181110010010111010 & Optimization Tim & ime $=100$ seconds & & 100 RWS & $4 \times 4$ & Single-point & 0.8 Random Gene Replacement & 0.2 \\
\hline 191110010010111010 & Optimization Tim & ime $=100$ seconds & & 100 RWS & $4 \times 4$ & Single-point & 0.8 Random Gene Replacement & 0.2 \\
\hline 201110010010111010 & Optimization Tim & ime $=50$ seconds & & 100 RWS & $4 \times 4$ & Single-point & 0.8 Random Gene Replacement & 0.2 \\
\hline 211110010010111010 & Optimization Tim & ime $=50$ seconds & & 100 RWS & $4 \times 4$ & Single-point & 0.8 Random Gene Replacement & 0.2 \\
\hline 2211100100101110100 & Optimization Tim & ime $=50$ seconds & & 100 RWS & $4 \times 4$ & Single-point & 0.8 Random Gene Replacement & 0.2 \\
\hline 2311100100101110100 & Optimization Tim & ime $=50$ seconds & & 100 RWS & $4 \times 4$ & Single-point & 0.8 Random Gene Replacement & 0.2 \\
\hline 2411100100101110100 & Optimization Tim & $\mathrm{ime}=50$ seconds & & 100 RWS & $4 \times 4$ & Single-point & 0.8 Random Gene Replacement & 0.2 \\
\hline 251110010010111010 & Optimization Tim & ime $=50$ seconds & & 100 RWS & $4 \times 4$ & Single-point & 0.8 Random Gene Replacement & 0.2 \\
\hline 261110010010111010 & Optimization Tim & ime $=50$ seconds & & 100 RWS & $4 \times 4$ & Single-point & 0.8 Random Gene Replacement & 0.2 \\
\hline 2711100100101110100 & Optimization Tim & ime $=50$ seconds & & 100 RWS & $4 \times 4$ & Single-point & 0.8 Random Gene Replacement & 0.2 \\
\hline 281110010010111010 & Optimization Tim & ime $=50$ seconds & & 100 RWS & $4 \times 4$ & Single-point & 0.8 Random Gene Replacement & 0.2 \\
\hline 2911100100101110100 & Optimization Tim & ime $=50$ seconds & & 100 RWS & $4 \times 4$ & Single-point & 0.8 Random Gene Replacement & 0.2 \\
\hline 3011100100101110100 & Optimization Tim & ime $=50$ seconds & & 100 RWS & $4 \times 4$ & Single-point & 0.8 Random Gene Replacement & 0.2 \\
\hline Starting Fitness & & Ending Fitr & ness & Generations & ution found & Difference & \# Trials Prematurely Conver & ged \\
\hline & 2610 & & 4510 & & 113356 & 1900 & & 1 \\
\hline & 2400 & & 5610 & & 115628 & 3210 & Average Fitness Gain & \\
\hline & 2460 & & 4460 & & 112806 & 2000 & & 2086 \\
\hline & 2250 & & 5210 & & 114680 & 2960 & Average Ending Fitness & \\
\hline & 3100 & & 4060 & & 99292 & 960 & & 4701 \\
\hline & 3020 & & 4090 & & 82190 & 1070 & Average Starting Fitness & \\
\hline & 3100 & & 5130 & & 55342 & $2030^{\prime}$ & & 2615 \\
\hline & 2460 & & 5180 & & 82336 & 2720 & & \\
\hline & 2450 & & 4860 & & 99691 & 2410 & & \\
\hline & 2300 & & 3900 & & 98482 & 1600 & & \\
\hline & 2460 & & 4060 & & 10457 & 1600 & \# Trials Prematurely Conver & ged \\
\hline & 2860 & & 4300 & & 9289 & 1440 & & 2 \\
\hline & 2460 & & 4220 & & 10729 & 1760 & Average Fitness Gain & \\
\hline & 2410 & & 4250 & & 9313 & 1840 & & 1520 \\
\hline & 2300 & & 4060 & & 9545 & 1760 & Average Ending Fitness & \\
\hline & 2890 & & 3930 & & 11522 & 1040 & & 4136 \\
\hline & 2570 & & 4810 & & 10368 & 2240 & Average Starting Fitness & \\
\hline & 2300 & & 3820 & & 10211 & $1520^{\prime}$ & & 2616 \\
\hline & 2810 & & 4010 & & 10599 & 1200 & & \\
\hline & 3100 & & 3900 & & 10428 & 800 & & \\
\hline & 3250 & & 4750 & & 4914 & 1500 & \# Trials Prematurely Conver & ged \\
\hline & 2620 & & 5180 & & 5183 & 2560 & & 2 \\
\hline & 4010 & & 5210 & & 5237 & 1200 & Average Fitness Gain & \\
\hline & 3420 & & 3420 & & 5226 & 0 & & 1393 \\
\hline & 3420 & & 4060 & & 5363 & 640 & Average Ending Fitness & \\
\hline & 2460 & & 3820 & & 5213 & $1360^{\prime}$ & & 4543 \\
\hline & 3260 & & 3260 & & 5157 & 0 & Average Starting Fitness & \\
\hline & 3260 & & 4460 & & 5270 & $1200^{\prime \prime}$ & & 3150 \\
\hline & 2300 & & 6010 & & 5350 & 3710 & & \\
\hline & 3500 & & 5260 & & 5471 & 1760 & & \\
\hline
\end{tabular}



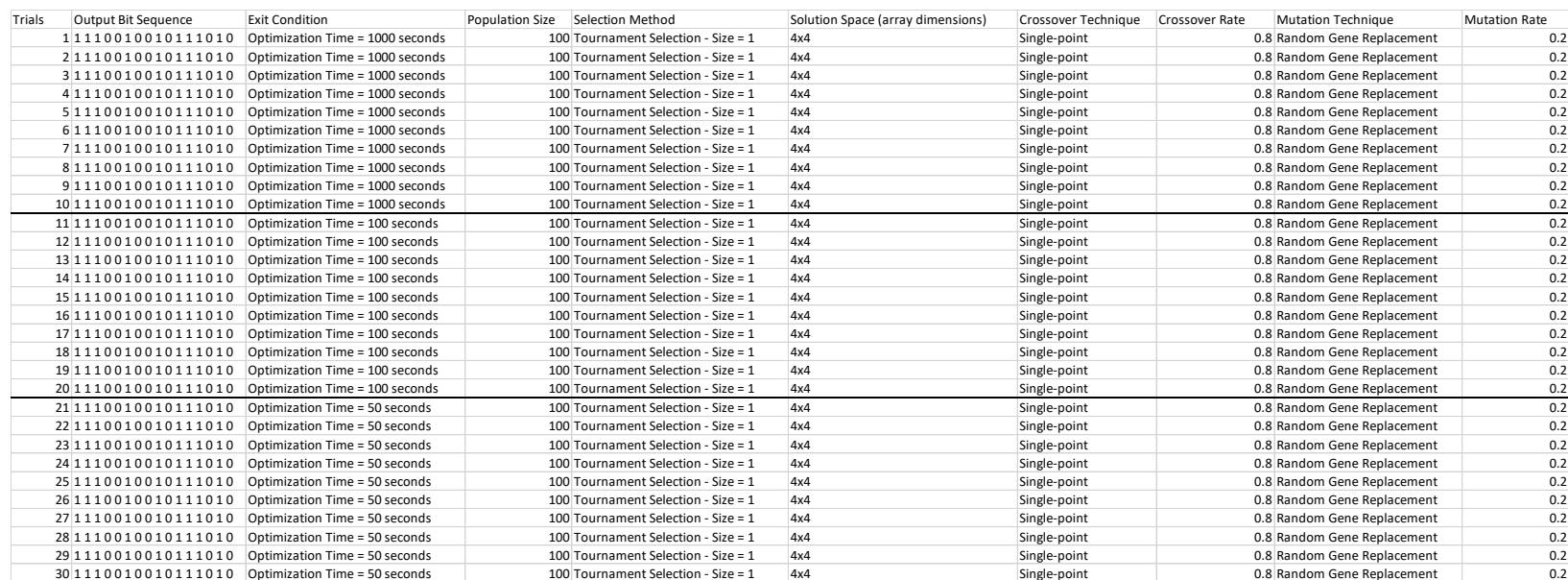

\begin{tabular}{|c|c|c|c|c|}
\hline Starting Fitness & Ending Fitness & Generations after solution found & Difference & \# Trials Prematurely Converged \\
\hline 2460 & 4940 & 114185 & 2480 & 2 \\
\hline 2460 & 4460 & 107259 & 2000 & Average Fitness Gain \\
\hline 2780 & 5260 & 110425 & 2480 & 2446 \\
\hline 2300 & 4700 & 108704 & 2400 & Average Ending Fitness \\
\hline 3210 & 3770 & 116533 & 560 & 5066 \\
\hline 2800 & 4270 & 113789 & 1470 & Average Starting Fitness \\
\hline 2570 & 6730 & 111817 & 4160 & 2620 \\
\hline 2460 & 5500 & 115805 & 3040 & \\
\hline 2460 & 6570 & 109764 & 4110 & \\
\hline 2700 & 4460 & 95741 & 1760 & \\
\hline 2250 & 5660 & 8729 & 3410 & \# Trials Prematurely Converged \\
\hline 2460 & 4780 & 9417 & 2320 & 5 \\
\hline 2300 & 4060 & 9294 & 1760 & Average Fitness Gain \\
\hline 3130 & 4810 & 9374 & 1680 & 1958 \\
\hline 3210 & 3820 & 9256 & 610 & Average Ending Fitness \\
\hline 2460 & 4060 & 9348 & 1600 & 4522 \\
\hline 2610 & 5100 & 7992 & 2490 & Average Starting Fitness \\
\hline 2300 & 5610 & 5658 & 3310 & 2564 \\
\hline 2460 & 3260 & 5034 & 800 & \\
\hline 2460 & 4060 & 4309 & 1600 & \\
\hline 2410 & 4010 & 2162 & 1600 & \# Trials Prematurely Converged \\
\hline 3050 & 5500 & 4227 & 2450 & 5 \\
\hline 2620 & 5610 & 3204 & 2990 & Average Fitness Gain \\
\hline 2650 & 2810 & 3314 & 160 & 1906 \\
\hline 3260 & 4620 & 3973 & 1360 & Average Ending Fitness \\
\hline 2620 & 5020 & 8619 & 2400 & 4618 \\
\hline 2450 & 5180 & 4375 & 2730 & Average Starting Fitness \\
\hline 2460 & 4060 & 4567 & 1600 & 2712 \\
\hline 2450 & 4220 & 4764 & 1770 & \\
\hline 3150 & 5150 & 4846 & 2000 & \\
\hline
\end{tabular}



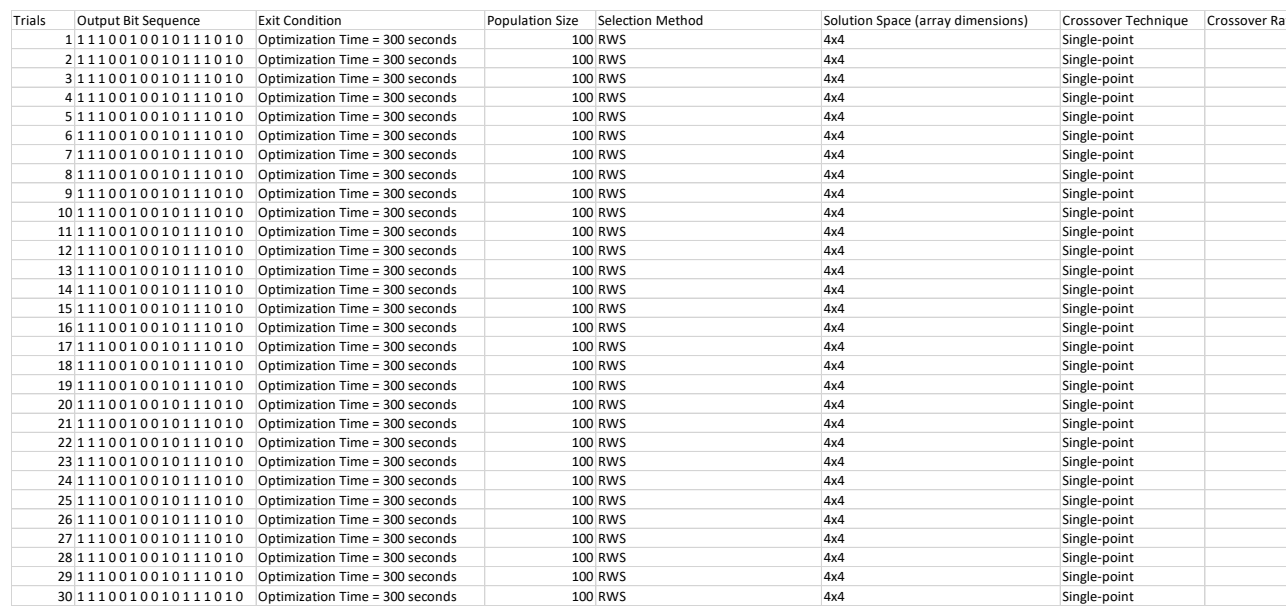

Mutation Technique

0.5 Random Gene Replacement 0.5 Random Gene Replacement 0.5 Random Gene Replacement 0.5 Random Gene Replacement 0.5 Random Gene Replacemen 0.5 Random Gene Replacement 0.5 Random Gene Replacement 0.5 Random Gene Replacement 0.5 Random Gene Replacement 0.5 Random Gene Replacement 0.5 Random Gene Replacement 0.5 Random Gene Replacement 0.5 Random Gene Replacement 0.5 Random Gene Replacemen 0.5 Random Gene Replacement 0.5 Random Gene Replacement 0.5 Random Gene Replacement 0.5 Random Gene Replocement 0.5 Random Gene Replacement 0.5 Random Gene Replacement 0.5 Random Gene Replacemen 0.5 Random Gene Replacement 0.5 Random Gene Replacement 0.5 Random Gene Replacement 0.5 Random Gene Replacemen 0.5 Random Gene Replacement 100 RWS Single-point
Single-point

\begin{tabular}{|c|c|c|c|c|}
\hline Starting Fitness & Ending Fitness & Generations after solution found & Difference & \# Trials Prematurely Converged \\
\hline 2300 & 4220 & 29639 & 1920 & 0 \\
\hline 2450 & 4110 & 29496 & 1660 & \\
\hline 2810 & 6170 & 29806 & 3360 & \\
\hline 2940 & 3690 & 19882 & 750 & \\
\hline 2410 & 3660 & 17381 & 1250 & \\
\hline 2700 & 5610 & 17200 & 2910 & \\
\hline 3580 & 5180 & 28781 & 1600 & \\
\hline 2620 & 4330 & 26806 & 1710 & \\
\hline 3660 & 4460 & 29461 & 800 & \\
\hline 2300 & 6060 & 29475 & 3760 & \\
\hline 2620 & 4460 & 27971 & 1840 & \\
\hline 3420 & 4910 & 28721 & 1490 & \\
\hline 3470 & 5310 & 29441 & 1840 & \\
\hline 2620 & 5130 & 26438 & 2510 & \\
\hline 2460 & 4110 & 25702 & 1650 & \\
\hline 2300 & 4300 & 24764 & 2000 & \\
\hline 2770 & 4270 & 29046 & 1500 & \\
\hline 3260 & 4510 & 26512 & 1250 & \\
\hline 2810 & 4010 & 26650 & 1200 & \\
\hline 2620 & 4430 & 28362 & 1810 & \\
\hline 3660 & 4620 & 24384 & 960 & \\
\hline 3100 & 5070 & 21255 & 1970 & \\
\hline 2860 & 4060 & 21313 & 1200 & \\
\hline 4060 & 4460 & 24142 & 400 & \\
\hline 3150 & 5070 & 17684 & 1920 & \\
\hline 3370 & 4910 & 21305 & 1540 & \\
\hline 2860 & 4620 & 29713 & 1760 & \\
\hline 3260 & 4620 & 29469 & 1360 & \\
\hline 2890 & 4570 & 29346 & 1680 & \\
\hline 2300 & 6170 & 29410 & 3870 & \\
\hline 2921 & 4703.333333 & 25985.16667 & 1782.333333 & \\
\hline
\end{tabular}




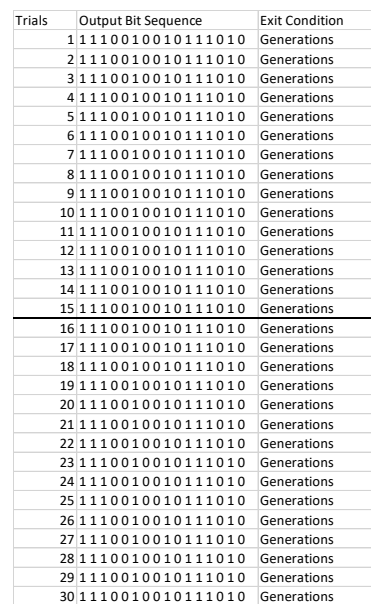

Starting Fitness
Population Size Selection Method 500 Tournament Selection - Size $=1$ 500 Tournament Selection - Size $=1$ 500 Tournament Selection - Size $=1$ 500 Tournament Selection - Size $=1$ 500 Tournament Selection - Size $=1$ 500 Tournament Selection - Size $=1$ 500 Tournament Selection - Size $=1$ 500 Tournament Selection - Size $=1$ 500 Tournament Selection - Size $=1$ 500 Tournament Selection - Size $=1$ 500 Tournament Selection - Size $=1$ 500 Tournament Selection - Size $=$ 50 Tournament Selection - Size $=1$ 50 Tournament Selection - Size $=1$ 50 Tournament Selection - Size $=1$ 50 Tournament Selection - Size $=1$ 50 Tournament Selection - Size $=1$ 50 Tournament Selection - Size $=1$ 50 Tournament Selection - Size $=1$ 50 Tournament Selection - Size $=1$ 50 Tournament Selection - Size $=1$ 50 Tournament Selection - Size $=1$ 50 Tournament Selection - Size $=1$ 50 Tournant Selection - Size $=$ 50 Tournament Selection - Size $=$

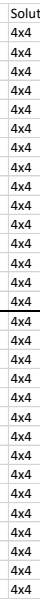

\begin{tabular}{l} 
Solutio \\
$4 \times 4$ \\
$4 \times 4$ \\
$4 \times 4$ \\
$4 \times 4$ \\
$4 \times 4$ \\
$4 \times 4$ \\
$4 \times 4$ \\
$4 \times 4$ \\
$4 \times 4$ \\
$4 \times 4$ \\
$4 \times 4$ \\
$4 \times 4$ \\
$4 \times 4$ \\
$4 \times 4$ \\
$4 \times 4$ \\
\hline $4 \times 4$ \\
$4 \times 44$ \\
$4 \times 4$ \\
$4 \times 4$ \\
$4 \times 4$ \\
$4 \times 44$ \\
$4 \times 4$ \\
$4 \times 4$ \\
$4 \times 4$ \\
$4 \times 4$ \\
$4 \times 4$ \\
$4 \times 4$ \\
$4 \times 4$ \\
$4 \times 4$ \\
$4 \times 4$
\end{tabular} Single-poin Single-poi
Single-poi Single-point Single-poin Single-point Single-poi single-poin Single-poin Single-poin Single-poin Single-poi Single-poin Single-po Single-point Single-point Single-poin Single-poi Single-poin Single-poin
Single-point Single-poin single-po single-point single-poin Single-poin Single-poin

Single-poin
Single-poin

\begin{tabular}{|c|c|c|c|c|c|}
\hline Starting Fitness & Ending Fitness & Generations after solution found & Difference & Computation Time & \# Trials Prematurely Converged \\
\hline 3450 & 6570 & 30000 & 3120 & 1215.1 & 0 \\
\hline 2250 & 7370 & 30000 & 5120 & 1213.398 & 8 Average Fitness Gain \\
\hline 3020 & 4620 & 30000 & 1600 & 1204.394 & 2940.666667 \\
\hline 3420 & 6170 & 30000 & 2750 & 1228.438 & 3 Average Ending Fitness \\
\hline 2610 & 5500 & 30000 & 2890 & 1232.103 & 5699.333333 \\
\hline 2460 & 5370 & 30000 & 2910 & 1386.38 & Average Starting Fitness \\
\hline 2460 & 4890 & 30000 & 2430 & 1559.646 & 2758.666667 \\
\hline 3210 & 6060 & 30000 & 2850 & 1180.885 & 5 Average Computation Time \\
\hline 2410 & 6410 & 30000 & 4000 & 1194.222 & 1258.4928 \\
\hline 2300 & 5820 & 30000 & 3520 & 1170.632 & \\
\hline 2250 & 4570 & 30000 & 2320 & 1211.707 & \\
\hline 3050 & 6810 & 30000 & 3760 & 1269.043 & \\
\hline 3420 & 4860 & 30000 & 1440 & 1228.105 & \\
\hline 2770 & 5260 & 30000 & 2490 & 1386.321 & \\
\hline 2300 & 5210 & 30000 & 2910 & 1197.018 & \\
\hline 3260 & 4220 & 30000 & 960 & 160.658 & 8 \# Trials Prematurely Converged \\
\hline 2300 & 5500 & 30000 & 3200 & 153.825 & 5 \\
\hline 2300 & 4110 & 30000 & 1810 & 151.848 & 3 Average Fitness Gain \\
\hline 4220 & 6620 & 30000 & 2400 & 148.702 & 2174.666667 \\
\hline 2650 & 5210 & 30000 & 2560 & 152.299 & Average Ending Fitness \\
\hline 2300 & 5020 & 30000 & 2720 & 153.065 & 4947.333333 \\
\hline 3020 & 4060 & 30000 & 1040 & 152.142 & Average Starting Fitness \\
\hline 2890 & 4570 & 30000 & 1680 & 177.304 & 2772.666667 \\
\hline 2450 & 5070 & 30000 & 2620 & 165.528 & 8 Average Computation Time \\
\hline 2300 & 4060 & 30000 & 1760 & 156.816 & 5" 156.1214 \\
\hline 2510 & 5100 & 30000 & 2590 & 150.693 & \\
\hline 2620 & 3980 & 30000 & 1360 & 151.603 & \\
\hline 3260 & 5770 & 30000 & 2510 & 152.313 & \\
\hline 2650 & 6060 & 30000 & 3410 & 151.725 & \\
\hline 2860 & 4860 & 30000 & 2000 & 163.3 & \\
\hline
\end{tabular}




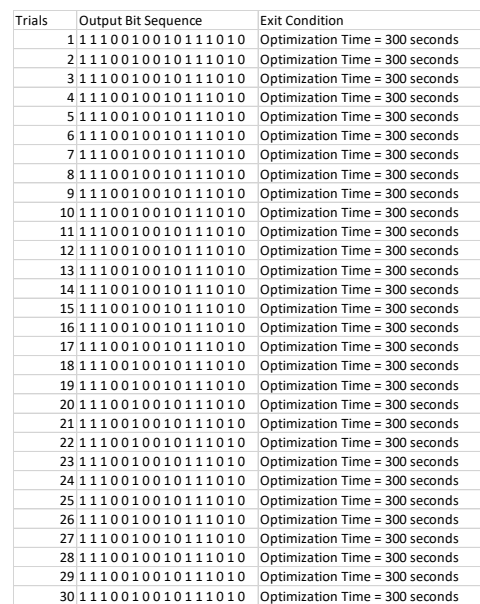

Population Size Selection Method 100 Tournament Selection - Size $=1$ 100 Tournament Selection - Size $=$ 100 Tournament Selection - Size $=1$ 100 Tournament Selection - Size $=1$ 100 Tournament Selection - Size $=1$ 100 Tournament Selection - Size $=1$ 100 Tournament Selection - Size $=1$ 100 Tournament Selection - Size $=1$ 100 Tournament Selection - Size $=1$ 100 Tournament Selection - Size $=1$ 100 Tournament Selection - Size $=1$ 100 Tournament Selection - Size $=1$ 100 Tournament Selection - Size $=1$ 100 Tournament Selection - Size $=1$ 100 Tournament Selection - Size $=1$ 100 Tournament Selection - Size $=1$ 100 Tournament Selection - Size $=1$ 100 Tournament Selection - Size $=1$ 100 Tournament Selection - Size $=1$ 100 Tournament Selection - Size $=1$ 100 Tournament Selection - Size $=1$ 100 Tournament Selection - Size $=1$ 100 Tournament Selection - Size $=1$ 100 Tournament Selection- Size $=1$ 100 Tournament Selection-Size = 100 Tournament Selection - Size $=1$

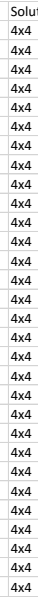

Solution Space (array dimensions)
$4 \times 4$
$4 \times 4$
$4 \times 4$
$4 \times 4$
$4 \times 4$
$4 \times 4$
$4 \times 4$
$4 \times 4$
$4 \times 4$
$4 \times 4$
$4 \times 4$
$4 \times 4$
$4 \times 4$
$4 \times 4$
$4 \times 4$
$4 \times 4$
$4 \times 4$
$4 \times 4$
$4 \times 4$
$4 \times 4$
$4 \times 4$
$4 \times 4$
$4 \times 4$
$4 \times 4$
$4 \times 4$
$4 \times 4$
$4 \times 4$
$4 \times 4$
$4 \times 4$
$4 \times 4$

Crossover Technique Crossover Rate
Uniform (rate $=0.5$ )
Uniform (rate $=0.5$ )
Uniform (rate $=0.5$ )
Uniform (rate $=0.5$ )
Uniform (rate $=0.5$ )
Uniform (rate $=0.5$ )
Uniform (rate $=0.5$ )
Uniform (rate $=0.5)$
Uniform (rate $=0.5$ )
Uniform (rate $=0.5$ )
Uniform (rate $=0.5$ )
Uniform (rate $=0.5$ )
Uniform (rate $=0.5$ )
Uniform (rate $=0.5$ )
Uniform (rate $=0.5$ )
Uniform (rate $=0.5$ )
Uniform (rate $=0.5$ )
Uniform (rate $=0.5$ )
Uniform (rate $=0.5$ )
Uniform (rate $=0.5$ )
Uniform (rate $=0.5$ )
Uniform (rate $=0.5$ )
Uniform (rate $=0.5$ )
Uniform (rate $=0.5$ )
Uniform (rate $=0.5$
Uniform (rate $=0.5$ )
Uniform (rate $=0.5$ )
Uniform (rate $=0.5$ )
Uniform (rate $=0.5$ )
Unifom (rate 0.5 )

Mutation Technique 0.8 Random Gene Replacemen 0.8 Random Gene Replacement 0.8 Random Gene Replacement 0.8 Random Gene Replacement 0.8 Random Gene Replacement 0.8 Random Gene Replacement 0.8 Random Gene Replacement 0.8 Random Gene Replacement 08 Pandom Gene Replacement 0.8 Random Gene Replacement 0.8 Random Gene Replacement 0.8 Random Gene Replacement 0.8 Random Gene Replacement 0.8 Random Gene Replacemen 0.8 Random Gene Replacement 0.8 Random Gene Replacement 0.8 Random Gene Replacemen 0.8 Random Gene Replacement 0.8 Random Gene Replacement 0.8 Random Gene Replacement 0.8 Random Gene Replacement 0.8 Random Gene Replacement 0.8 Random Gene Replacement 0.8 Random Gene Replacement 0.8 Random Gene Replacement 08 Random Gene Replacement

Mutation Rate

\# Trials Prematurely Converged

\begin{tabular}{|c|c|c|c|c|}
\hline Starting Fitness & Ending Fitness & Generations after solution found & Difference & \# Trials Prematurely Converged \\
\hline 2250 & 4570 & 31130 & 2320 & 3 \\
\hline 2250 & 5370 & 31750 & 3120 & \\
\hline 3260 & 5550 & 31395 & 2290 & \\
\hline 2460 & 4860 & 30852 & 2400 & \\
\hline 3260 & 5050 & 29332 & 1790 & \\
\hline 2650 & 3100 & 30081 & 450 & \\
\hline 2450 & 4510 & 29830 & 2060 & \\
\hline 2610 & 5740 & 33939 & 3130 & \\
\hline 2620 & 5660 & 29376 & 3040 & \\
\hline 2860 & 5020 & 30384 & 2160 & \\
\hline 3260 & 5180 & 20938 & 1920 & \\
\hline 2460 & 4250 & 32962 & 1790 & \\
\hline 3250 & 4750 & 33929 & 1500 & \\
\hline 2300 & 5900 & 33763 & 3600 & \\
\hline 3260 & 4460 & 24755 & 1200 & \\
\hline 2700 & 4060 & 30194 & 1360 & \\
\hline 3260 & 5180 & 30980 & 1920 & \\
\hline 2460 & 6060 & 30466 & 3600 & \\
\hline 3210 & 5580 & 29664 & 2370 & \\
\hline 2450 & 5850 & 24964 & 3400 & \\
\hline 2700 & 5900 & 22943 & 3200 & \\
\hline 3410 & 5610 & 32157 & 2200 & \\
\hline 2460 & 5210 & 30155 & 2750 & \\
\hline 3260 & 5740 & 34346 & 2480 & \\
\hline 3100 & 5020 & 34360 & 1920 & \\
\hline 3500 & 4700 & 34044 & 1200 & \\
\hline 3850 & 5500 & 34680 & 1650 & \\
\hline 2700 & 4060 & 33392 & 1360 & \\
\hline 2670 & 5420 & 34289 & 2750 & \\
\hline 2450 & 4620 & 34027 & 2170 & \\
\hline 2846 & 5082.666667 & 30835.9 & 2236.666667 & \\
\hline
\end{tabular}




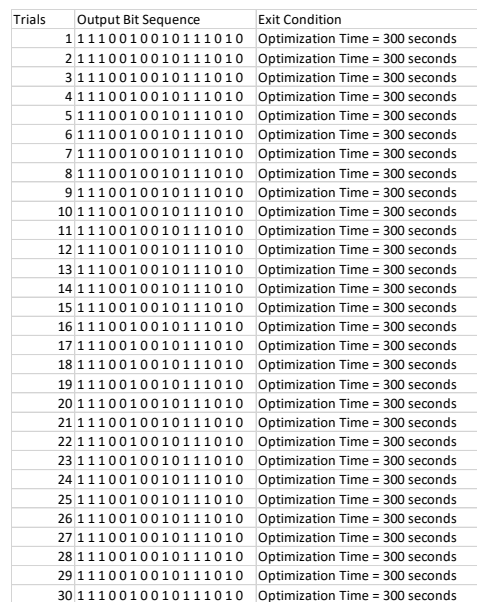

Population Size Selection Method 100 Tournament Selection - Size $=1$ 100 Tournament Selection - Size $=1$ 100 Tournament Selection - Size $=1$ 100 Tournament Selection - Size $=1$ 100 Tournament Selection - Size = 100 Tournament Selection - Size $=$ 100 Tournament Selection - Size $=1$ 100 Tournament Selection - Size $=1$ 100 Tournament Selection - Size $=1$ 100 Tournament Selection - Size $=1$ 100 Tournament Selection - Size $=1$ 100 Tournament Selection - Size $=1$ 100 Tournament Selection - Size $=1$ 100 Tournament Selection - Size $=1$ 100 Tournament Selection - Size $=1$ 100 Tournament Selection - Size $=1$ 100 Tournament Selection - Size $=1$ 100 Tournament Selection - Size $=1$ 100 Tournament Selection - Size $=1$ 100 Tournament Selection - Size $=1$ 100 Tournament Selection - Size $=$ 100 Tournament Selection - Size 100 Tournament Selection - Size $=1$ 100 Tournament Selection- Size $=1$ 100 Tournament Selection-Size = 100 Tournament Selection - Size $=1$

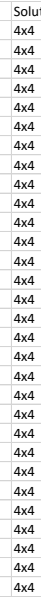

Solution Space (array dimensions)
$4 \times 4$
$4 \times 4$
$4 \times 4$
$4 \times 4$
$4 \times 4$
$4 \times 4$
$4 \times 4$
$4 \times 4$
$4 \times 4$
$4 \times 4$
$4 \times 4$
$4 \times 4$
$4 \times 4$
$4 \times 4$
$4 \times 4$
$4 \times 4$
$4 \times 4$
$4 \times 4$
$4 \times 4$
$4 \times 4$
$4 \times 4$
$4 \times 4$
$4 \times 4$
$4 \times 4$
$4 \times 4$
$4 \times 4$
$4 \times 4$
$4 \times 4$
$4 \times 4$
$4 \times 4$

Crossover Technique Crossover Rate
Uniform (rate $=0.5$ )
Uniform (rate $=0.5$ )
Uniform (rate $=0.5$ )
Uniform (rate $=0.5$ )
Uniform (rate $=0.5$ )
Uniform (rate $=0.5$ )
Uniform (rate $=0.5$ )
Uniform (rate $=0.5)$
Uniform (rate $=0.5$ )
Uniform (rate $=0.5$ )
Uniform (rate $=0.5$ )
Uniform (rate $=0.5$ )
Uniform (rate $=0.5$ )
Uniform (rate $=0.5$ )
Uniform (rate $=0.5$ )
Uniform (rate $=0.5$ )
Uniform (rate $=0.5$ )
Uniform (rate $=0.5$ )
Uniform (rate $=0.5$ )
Uniform (rate $=0.5$ )
Uniform (rate $=0.5$ )
Uniform (rate $=0.5$ )
Uniform (rate $=0.5$ )
Uniform (rate $=0.5$ )
Uniform (rate $=0.5$
Uniform (rate $=0.5$ )
Uniform (rate $=0.5$ )
Uniform (rate $=0.5$ )
Uniform (rate $=0.5$ )
Unifom (rate 0.5 )

Mutation Technique 0.5 Random Gene Replacemen 0.5 Random Gene Replacement 0.5 Random Gene Replacement 0.5 Random Gene Replacement 0.5 Random Gene Replacement 0.5 Random Gene Replacemen 0.5 Random Gene Replacement 0.5 Random Gene Replacement 0.5 Pandom Gene Replacement 0.5 Random Gene Replacement 0.5 Random Gene Replacemen 0.5 Random Gene Replacement 0.5 Random Gene Replacemen 0.5 Random Gene Replacemen 0.5 Random Gene Replacement 0.5 Random Gene Replacement 0.5 Random Gene Replacement 0.5 Random Gene Replacemen 0.5 Random Gene Replacement 0.5 Random Gene Replacement 0.5 Random Gene Replacement 0.5 Random Gene Replacemen 0.5 Random Gene Replacement 0.5 Random Gene Replacement 0.5 Random Gene Replacement 0.5 Random Gene Replacemen Generations after solution found

Difference

\# Trials Prematurely Converged

\begin{tabular}{|c|c|c|c|c|}
\hline Starting Fitness & Ending Fitness & Generations after solution found & Difference & \# Trials Prematurely Converged \\
\hline 2450 & 5020 & 28985 & 2570 & 0 \\
\hline 2610 & 4270 & 31940 & 1660 & \\
\hline 2450 & 5310 & 24796 & 2860 & \\
\hline 2410 & 6620 & 24103 & 4210 & \\
\hline 2620 & 3610 & 26160 & 990 & \\
\hline 2610 & 4700 & 26903 & 2090 & \\
\hline 2460 & 5260 & 26311 & 2800 & \\
\hline 3260 & 5260 & 31105 & 2000 & \\
\hline 2960 & 4730 & 31021 & 1770 & \\
\hline 2810 & 6330 & 31069 & 3520 & \\
\hline 2300 & 5050 & 28920 & 2750 & \\
\hline 2610 & 5820 & 24021 & 3210 & \\
\hline 2460 & 6060 & 27175 & 3600 & \\
\hline 2450 & 4750 & 31458 & 2300 & \\
\hline 4110 & 5070 & 32846 & 960 & \\
\hline 2450 & 5500 & 33165 & 3050 & \\
\hline 2650 & 3660 & 28446 & 1010 & \\
\hline 2610 & 6090 & 33327 & 3480 & \\
\hline 2610 & 5420 & 33151 & 2810 & \\
\hline 2460 & 4060 & 33343 & 1600 & \\
\hline 2450 & 5980 & 33401 & 3530 & \\
\hline 2300 & 5070 & 32820 & 2770 & \\
\hline 3250 & 4910 & 33228 & 1660 & \\
\hline 2400 & 3770 & 33107 & 1370 & \\
\hline 2300 & 4860 & 33040 & 2560 & \\
\hline 3020 & 4780 & 30288 & 1760 & \\
\hline 2460 & 4700 & 24531 & 2240 & \\
\hline 3100 & 4060 & 21012 & 960 & \\
\hline 2450 & 4060 & 18654 & 1610 & \\
\hline 3500 & 4460 & 30802 & 960 & \\
\hline 2686 & 4974.666667 & 29304.26667 & 2288.666667 & \\
\hline
\end{tabular}




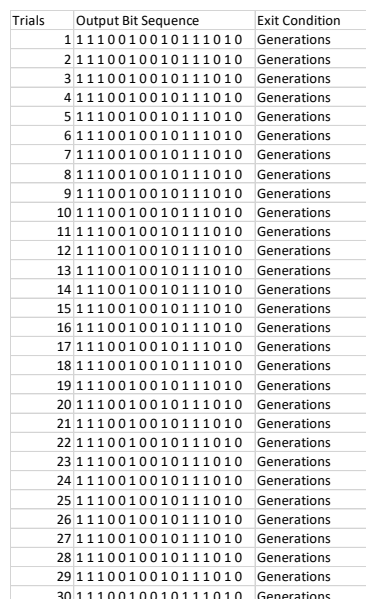

Population Size Selection Method 500 RWS 500 RWS 500 RWS 500 RWS 500 RWS 500 RWS 500 RWS 500 RWS 500 RWS 500 RWS 500 RWS 500 RWS 500 RWS 500 RWS 500 RWS 500 RWS 500 RWS 500 RWS
500 RWS 500 RWS 500 RWS 500 RWS
500 RWS 500 RWS 500 RWS 500 RWS 500 RWS 500 RWS

Starting Fitness Ending Fitness Generations after solution found Difference

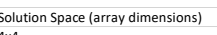

Solution

$4 \times 4$
$4 \times 4$

$4 \times 4$

$4 \times 4$
$4 \times 4$
$4 \times 4$

$4 \times 4$

$\times 4$

$4 \times 4$

$\times 4$

$4 \times 4$

$4 \times 4$

$4 \times 4$
$4 \times 4$
$4 \times 4$

$4 \times 4$

$4 \times 4$

$4 \times 4$

$4 \times 4$

$4 \times 4$

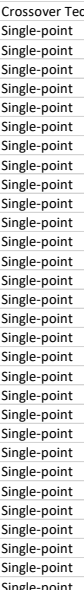

Crossover Rate

Mutation Technique

0.8 Random Gene Replacement

0.8 Random Gene Replacement 0.8 Random Gene Replacement 0.8 Random Gene Replacement 0.8 Random Gene Replacement 0.8 Random Gene Replacement 0.8 Random Gene Replacement 0.8 Random Gene Replacement 0.8 Random Gene Replacement 0.8 Random Gene Replacement 0.8 Random Gene Replacement 0.8 Random Gene Replacemen 0.8 Random Gene Replacement 0.8 Random Gene Replacement 0.8 Random Gene Replacement 0.8 Random Gene Replacement 0.8 Random Gene Replacement 0.8 Random Gene Replacemen 0.8 Random Gene Replacement 0.8 Random Gene Replacemen 0.8 Random Gene Replacemen 0.8 Random Gene Replacement 0.8 Random Gene Replacement 0.8 Random Gene Replacement 0.8 Random Gene Replacemen 0.8 Random Gene Replacement

\begin{tabular}{|c|c|c|c|c|c|}
\hline Starting Fitness & Ending Fitness & Generations after solution found & Difference & Computation Time & \# Trials Prematurely Converged \\
\hline 2410 & 4170 & 30000 & 1760 & 1529.3 & 1 \\
\hline 2460 & 4220 & 30000 & 1760 & 1621.248 & \\
\hline 3420 & 4860 & 30000 & 1440 & 1,352 & \\
\hline 3210 & 5850 & 30000 & 2640 & 1232.35 & \\
\hline 2670 & 5660 & 30000 & 2990 & 1245.1 & \\
\hline 3120 & 6650 & 30000 & 3530 & 1230.4 & \\
\hline 2460 & 4860 & 30000 & 2400 & 1243.1 & \\
\hline 2460 & 4060 & 30000 & 1600 & 1247.3 & \\
\hline 3100 & 4300 & 30000 & 1200 & 1256.3 & \\
\hline 2460 & 4700 & 30000 & 2240 & 1493.4 & \\
\hline 2650 & 4860 & 30000 & 2210 & 1341.8 & \\
\hline 3820 & 4860 & 30000 & 1040 & 1347.5 & \\
\hline 4220 & 4860 & 30000 & 640 & 1273.6 & \\
\hline 2410 & 4270 & 30000 & 1860 & 1374.1 & \\
\hline 2610 & 6090 & 30000 & 3480 & 1730.5 & \\
\hline 2300 & 4380 & 30000 & 2080 & 1458.7 & \\
\hline 2300 & 4300 & 30000 & 2000 & 1443.2 & \\
\hline 2610 & 5310 & 30000 & 2700 & 1494.6 & \\
\hline 2610 & 4110 & 30000 & 1500 & 1407.6 & \\
\hline 3200 & 5310 & 30000 & 2110 & 1318.8 & \\
\hline 2410 & 4220 & 30000 & 1810 & 1999.8 & \\
\hline 3260 & 4810 & 30000 & 1550 & 1420.5 & \\
\hline 3180 & 4220 & 30000 & 1040 & 1178.1 & \\
\hline 2460 & 4060 & 30000 & 1600 & 1155.4 & \\
\hline 2460 & 5420 & 30000 & 2960 & 1569.7 & \\
\hline 2460 & 4860 & 30000 & 2400 & 1286.4 & \\
\hline 2410 & 5930 & 30000 & 3520 & 1355.8 & \\
\hline 2700 & 4700 & 30000 & 2000 & 1264.9 & \\
\hline 2860 & 4620 & 30000 & 1760 & 1375.1 & \\
\hline 2450 & 4700 & 30000 & 2250 & 1190.6 & \\
\hline 2771.666667 & 4840.666667 & 30000 & 2069 & 1381.239933 & \\
\hline
\end{tabular}

